CERN-PH-TH/2006-108

LNF-06-16-P

$\mathrm{UCLA} / 06 / \mathrm{TEP} / 18$

\title{
Charge Orbits of Symmetric Special Geometries and Attractors
}

\author{
Stefano Bellucci, Sergio Ferrara ${ }^{\curvearrowright b}$, Murat Günaydin^ \\ and Alessio Marrani ${ }^{\bigcirc}$ \\ \& INFN - Laboratori Nazionali di Frascati, \\ Via Enrico Fermi 40,00044 Frascati, Italy \\ bellucci, marrani@lnf. infn. it \\ $\diamond$ Physics Department, Theory Unit, CERN, \\ CH 1211, Geneva 23, Switzerland \\ sergio.ferrara@cern.ch \\ $b$ Department of Physics and Astronomy, \\ University of California, Los Angeles, CA USA \\ ferrara@physics.ucla.edu \\ \$ Department of Physics, Penn State University \\ University Park, PA16802, USA \\ murat@phys.psu.edu \\ $\odot$ Museo Storico della Fisica e \\ Centro Studi e Ricerche "Enrico Fermi" \\ Via Panisperna 89A, 00184 Roma, Italy
}

\section{ABSTRACT}

We study the critical points of the black hole scalar potential $V_{B H}$ in $N=2, d=4$ supergravity coupled to $n_{V}$ vector multiplets, in an asymptotically flat extremal black hole background described by a $2\left(n_{V}+1\right)$-dimensional dyonic charge vector and (complex) scalar fields which are coordinates of a special Kähler manifold.

For the case of homogeneous symmetric spaces, we find three general classes of regular attractor solutions with non-vanishing Bekenstein-Hawking entropy. They correspond to three (inequivalent) classes of orbits of the charge vector, which is in a $2\left(n_{V}+1\right)$ dimensional representation $R_{V}$ of the $U$-duality group. Such orbits are non-degenerate, namely they have non-vanishing quartic invariant (for rank-3 spaces). Other than the $\frac{1}{2}$-BPS one, there are two other distinct non-BPS classes of charge orbits, one of which has vanishing central charge.

The three species of solutions to the $N=2$ extremal black hole attractor equations give rise to different mass spectra of the scalar fluctuations, whose pattern can be inferred by using invariance properties of the critical points of $V_{B H}$ and some group theoretical considerations on homogeneous symmetric special Kähler geometry. 


\section{Contents}

1 Introduction 1

2 BPS and non-BPS Attractors : Charge Orbits of $N=2, d=4$ MESGTS 6

3 Freudenthal Triple Systems and Orbits of Symmetric $N=2, d=4$ MESGTs 11

4 Classification of Attractors $\quad 27$

$4.1 \quad \frac{1}{2}$-BPS solutions . . . . . . . . . . . . . . . . . . . 27

4.2 Non-BPS solutions . . . . . . . . . . . . . . . . . 27

4.2.1 Non-BPS, $Z \neq 0$ solutions . . . . . . . . . . . . . . . . . 30

4.2.2 Non-BPS, $Z=0$ solutions . . . . . . . . . . . . . . . . . 32

4.3 Orbits and Attractors of $J_{2}^{\mathbb{D}}$ and $J_{2}^{\mathbb{H}} \ldots \ldots \ldots$. . . . . . . . . . . . 33

4.3.1 $N=2.6$ SUGRAs and the dual role of $\frac{S O^{*}(12)}{U(0)}$. . . . . . . . . 34

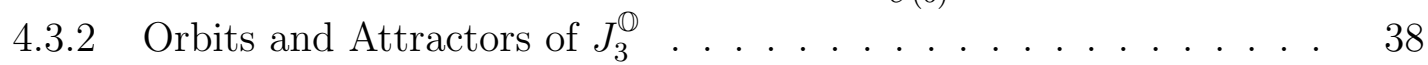

4.3 .3 Orbits and Attractors of $J_{3}^{\mathbb{H}}$. . . . . . . . . . . . . . . . . 40

5 The Mass Spectra at Critical Points 442

$\begin{array}{lll}6 & \text { Conclusion } & 48\end{array}$

A Appendix I The sequence $\frac{S U(1,1+n)}{U(1)}$

B Appendix II The sequence $\frac{S U(1,1)}{U(1)} \otimes \frac{S O(2,2+n)}{S(2)(2)+2(2+n)}$

\section{Introduction}

The charge configurations of stationary, spherically symmetric, asymptotically flat "classical" extremal black holes in $d=4$ dimensions are expressed by the (electric-magnetic field strengths) representation $R_{V}$ of the duality group $G_{4} \equiv G$ of the underlying $d=4$ supergravity (SUGRA) theory. It has been known for some time [1] that in the case of scalar manifolds which are symmetric spaces such "charge vectors" belong to distinct classes of orbits of the representation $R_{V}$, i.e. that the $R_{V}$-representation space of $G$ can be actually divided in disjoint classes of orbits. Such orbits are classified by suitable constraints on the (lowest order) $G$-invariant quantity $\mathcal{I}$ built out of the representation $R_{V}$.

Moreover, for $N \geqslant 3, d=4$ SUGRAs the scalar manifold of the theory is an homogeneous symmetric space $G / H$, and $R_{V}$ is a real symplectic representation of $G$. Thus, for such SUGRAs some relations between the coset expressions of the aforementioned orbits and different real (non-compact) forms of the stabilizer $H$ of the scalar manifold $G / H$ 
can be established (even though in the cases having $\mathcal{I}=0$ such relations do not rely on a critical Attractor Mechanism).

The work of [1] on the classification of the orbits of U-duality ${ }^{1}$ groups in maximal supergravity and $N=2$ MESGTs in five and four dimensions also suggested that four dimensional U-duality groups may act as spectrum generating conformal symmetry groups in the charge spaces of the corresponding five dimensional theories. In [3] this idea was developed further and extended to the proposal that the 3 dimensional $U$ duality group $E_{8(8)}$ of maximal supergravity must similarly act as a spectrum generating quasiconformal symmetry group in the charge space of black hole solutions in four dimensions extended by an extra coordinate interpreted as black hole entropy. This proposal extends naturally to 3 -dimensional U-duality groups of $N=2$ MESGTs acting as spectrum generating quasiconformal symmetry groups in four dimensions [4. Remarkably, the quantization of the geometric quasiconformal action yields directly the minimal unitary representation of the corresponding U-duality group [5, 6, 7]. More recently it was conjectured that the indexed degeneracies of certain $N=8$ and $N=4$ BPS black holes are given by some automorphic forms related to the minimal unitary representations of the corresponding 3 dimensional U-duality groups [8]. Motivated by these results and conjectures stationary and spherically symmetric solutions of $N \geq 2$ supergravities with symmetric scalar manifolds were studied in 9]. By using the equivalence of four dimensional attractor flow with the geodesic motion on the scalar manifold of the corresponding three dimensional theory the authors of [9] argued further that the three-dimensional U-duality groups must act as spectrum generating symmetry groups for BPS black hole degeneracies in 4 dimensions.

In this paper we will study in full generality the critical points (generically referred to as attractors) of the black hole scalar potential $V_{B H}$ for all $N=2$ symmetric special geometries in $d=4$. These extrema describe the regular configurations (BPS as well as non-BPS) of $N=2,6,8$ SUGRAs, corresponding to a finite, non-vanishing quartic invariant $I=I_{4}$ and thus to extremal black holes with classical non-vanishing entropy $S_{B H} \neq 0$. The related orbits in the $R_{V}$ of the $d=4$ duality group $G$ will correspondingly be referred to as non-degenerate orbits. The attractor equations for BPS configurations were first studied in [10, 11, 12, 13. Flow Equations for the general case were given in [14, and recently non-BPS attractors have been found for $N=2$ and $N=8$ theories [15]-26].

Attractor solutions and their non-degenerate charge orbits in $d=5$ have been recently classified for the case of all rank-2 symmetric spaces in [27].

Let us start by considering $N=8, d=4$ SUGRA. For such a theory the duality group is the Cremmer and Julia's [28] one $G=E_{7(7)}$ and the stabilizer is $H=S U(8)$, which is at the same time the $\mathcal{R}$-symmetry group of the $N=8$ supersymmetry algebra

\footnotetext{
${ }^{1}$ Here $U$-duality is referred to as the "continuous" version, valid for large values of the charges, of the $U$-duality groups introduced by Hull and Townsend [2].
} 
and the maximal compact subgroup (m.c.s.) of $E_{7(7)}$. The dimension of the resulting real scalar manifold $\frac{E_{7(7)}}{S U(8)}$ is 70 . The complex antisymmetric central charge matrix $Z_{A B}$ $(A=1, \ldots, N=8)$ sits in the real symplectic, fundamental representation $R_{V}=\mathbf{5 6}$ of $E_{7(7)}$. The two classes of non-degenerate charge orbits of the $\mathbf{5 6}$ of $E_{7(7)}$ are classified by the unique Cartan-Cremmer-Julia quartic $E_{7(7)}$-invariant [29, 28, $\mathcal{I}=I_{4}$ constructed from the 56 of $E_{7(7)}$ [1]. Depending on $\operatorname{sgn}\left(I_{4}\right)$, one gets:

$$
\begin{aligned}
& I_{4}>0: \mathcal{O}_{\frac{1}{8}-B P S}=\frac{E_{7(7)}}{E_{6(2)}}=\frac{G}{\mathcal{H}_{0}} \quad \frac{1}{8} \text {-BPS; } \\
& I_{4}<0: \mathcal{O}_{n o n-B P S}=\frac{E_{7(7)}}{E_{6(6)}}=\frac{G}{\widehat{\mathcal{H}}_{0}} \quad \text { non-BPS. }
\end{aligned}
$$

The real dimension of both orbits is $\operatorname{dim}\left(E_{7}\right)-\operatorname{dim}\left(E_{6}\right)=55$. No other classes of non-degenerate orbits exist in this case; this is essentially related to the fact that no other real (non-compact) forms of $E_{6}$ exist in $E_{7(7)}$ beside $E_{6(2)}$ and $E_{6(6)}$. The $\frac{1}{8}$-BPS and non-BPS non-degenerate orbits correspond to the maximal (non-compact) subgroup of $E_{7(7)}$ to be $E_{6(2)} \otimes U(1)$ and $E_{6(6)} \otimes S O(1,1)$, respectively.

Actually, $N=8$ non-degenerate orbits turn out to be classified by 5 moduli-dependent parameters [30, 31, 12, 4 positive eigenvalues $\rho_{1}, \ldots, \rho_{4}$ and an overall phase $\varphi$. Indeed, by using the fact that under $S U(8)$ the symplectic, fundamental real representation $R_{V}$ of $E_{7(7)}$ decomposes as $\mathbf{5 6}=\mathbf{2 8}+\overline{\mathbf{2 8}}$, one can see $Z_{A B}$ as a complex basis in the $\mathbf{5 6}$. Consequently, $Z_{A B}$ can be skew-diagonalized [32] by performing an $S U(8)$ rotation ${ }^{2}$. Such a procedure corresponds to nothing but a change of reference frame in the 56representation space of $E_{7(7)}$ :

Generic frame : $Z_{A B}$

$$
\downarrow S U(8) \text { rotation }
$$

$$
\begin{aligned}
& \text { "Normal" frame : } Z_{A B, \text { normal }}=e^{i \varphi / 4}\left(\begin{array}{cccc}
\rho_{1} & & & \\
& \rho_{2} & & \\
& & \rho_{3} & \\
& & & \rho_{4}
\end{array}\right) \otimes \epsilon \text {, } \\
& \rho_{1}, \rho_{2}, \rho_{3}, \rho_{4}, \in \mathbb{R}^{+}, \varphi \in[0,8 \pi),
\end{aligned}
$$

where $\epsilon$ is the 2-dim. symplectic metric

$$
\epsilon \equiv\left(\begin{array}{cc}
0 & -1 \\
1 & 0
\end{array}\right)
$$

\footnotetext{
${ }^{2}$ Actually, such a skew-diagonalization procedure is nothing but an application of the Bloch-MessiahZumino Theorem 33, 34 to the case of $N=8, d=4$ SUGRA.
} 
By looking at $Z_{A B, n o r m a l}$, it is immediate to conclude that the overall symmetry of such a moduli-dependent skew-diagonal $8 \times 8$ complex matrix in a generic point of the real 70-dim. scalar manifold $\frac{E_{7(7)}}{S U(8)}$ is $(S U(2))^{4}$. Thence, quartic $E_{7(7)}$-invariant $I_{4}$ can be written in the "normal" frame as follows 35]:

$$
\begin{aligned}
& I_{4, \text { normal }}\left(\rho_{1}, \rho_{2}, \rho_{3}, \rho_{4}, \varphi\right)= \\
& =\left[\left(\rho_{1}+\rho_{2}\right)^{2}-\left(\rho_{3}+\rho_{4}\right)^{2}\right]\left[\left(\rho_{1}-\rho_{2}\right)^{2}-\left(\rho_{3}-\rho_{4}\right)^{2}\right]+8 \rho_{1} \rho_{2} \rho_{3} \rho_{4}(\cos \varphi-1) .
\end{aligned}
$$

$N=8$ extremal black hole attractor equations [26] have only 2 distinct classes of regular solutions as expected from the analysis of [1]:

\section{1. $\frac{1}{8}$-BPS solution:}

$$
\rho_{1}=\rho_{\frac{1}{8}-B P S} \in \mathbb{R}_{0}^{+}, \varphi \in[0,8 \pi), \rho_{2}=\rho_{3}=\rho_{4}=0 .
$$

As given by Eq. (1.1), the corresponding orbit in the 56 of $E_{7(7)}$ is $\mathcal{O}_{\frac{1}{8}-B P S}=\frac{E_{7(7)}}{E_{6(2)}}$, with $I_{4, \text { normal }, \frac{1}{8}-B P S}=\rho_{\frac{1}{8}-B P S}^{4}>0$ and classical entropy given by the Bekenstein-Hawking entropy-area formula 36

$$
S_{B H, \frac{1}{8}-B P S}=\pi \sqrt{I_{4, \text { normal }, \frac{1}{8}-B P S}}=\pi \rho_{\frac{1}{8}-B P S}^{2}
$$

\section{2. non-BPS solution:}

$$
\rho_{1}=\rho_{2}=\rho_{3}=\rho_{4}=\rho_{\text {non-BPS }} \in \mathbb{R}_{0}^{+}, \quad \varphi=\pi .
$$

As given by Eq. (1.2), the corresponding orbit in the 56 of $E_{7(7)}$ is $\mathcal{O}_{n o n-B P S}=\frac{E_{7(7)}}{E_{6(6)}}$, with $I_{4, \text { normal,non-BPS }}=-16 \rho_{\text {non-BPS }}^{4}<0$ and classical entropy

$$
S_{B H, n o n-B P S}=\pi \sqrt{-I_{4, \text { normal,non-BPS }}}=4 \pi \rho_{\text {non-BPS }}^{2} .
$$

The deep meaning of the extra factor 4 in Eq. (1.9) as compared with Eq. (1.7) can be clearly explained when considering the so-called "stu interpretation" of $N=8$ regular critical points [26].

It is interesting to note that the symmetry gets enhanced at the particular points of $\frac{E_{7(7)}}{S U(8)}$ given by the aforementioned regular solutions. In general, the invariance properties of the regular solutions to attractor eqs. are given by the m.c.s. of the stabilizer of the corresponding charge orbit. In the considered case $N=8$, at $\frac{1}{8}$-BPS and non-BPS critical point(s) the following symmetry enhancements respectively hold:

$$
\begin{aligned}
& \frac{1}{8} \text {-BPS : }(S U(2))^{4} \longrightarrow S U(2) \otimes S U(6)=h_{0}=\text { m.c.s. }\left(\mathcal{H}_{0}=E_{6(2)}\right) \\
& \text { non-BPS : }(S U(2))^{4} \longrightarrow U S p(8)=\widehat{h}_{0}=\text { m.c.s. }\left(\widehat{\mathcal{H}}_{0}=E_{6(6)}\right)
\end{aligned}
$$


The aim of the present work is to extend the results holding for $N=8, d=4$ SUGRA to the particular class of $N=2, d=4$ symmetric Maxwell-Einstein SUGRA theories (MESGTs) [37, 38, 39]. Such a class consists of $N=2, d=4$ SUGRAs sharing the following properties:

i) beside the SUGRA multiplet, the matter content is given only by a certain number $n_{V}$ of Abelian vector multiplets;

ii) the space of the vector multiplets' scalars is an homogeneous symmetric special Kähler manifold, i.e. a special Kähler manifold with coset structure $\frac{G}{H_{0} \otimes U(1)}$, where $G$ is a semisimple non-compact Lie group and $H_{0} \otimes U(1)$ is its m.c.s.;

iii) the charge vector in a generic (dyonic) configuration with $n_{V}+1$ electric and $n_{V}+1$ magnetic charges sits in a real (symplectic) representation $R_{V}$ of $G$ of $\operatorname{dim}\left(R_{V}\right)=$ $2\left(n_{V}+1\right)$.

By exploiting such special features and relying on group theoretical considerations, we will be able to relate the coset expressions of the various distinct classes of nondegenerate orbits (of dimension $2 n_{V}+1$ ) in the $R_{V}$-representation space of $G$ to different real (non-compact) forms of the compact group $H_{0}$. Correspondingly, we will solve the $N=2$ extremal black hole attractor eqs. for all such classes, also studying the scalar mass spectrum of the theory corresponding to the obtained solutions.

The plan of the paper is as follows.

In Sect. 2 we review some basic facts about $N=2, d=4$ symmetric MESGTs, their non-degenerate charge orbits and the relations with the regular solutions of the $N=2$ extremal black hole attractor equations.

In Sect. 3 we determine the orbits of the U-duality groups of $N=2$ MESGTs whose scalar manifolds are symmetric spaces, acting on the representation $R_{V}$ of charges. With the exception of the $\frac{S U(1, n+1)}{S U(1+n) \otimes U(1)}$ family, all such MESGTs have their origin in fivedimensional $N=2$ MESGTs defined by Euclidean Jordan algebras of degree three. The five-dimensional correspondence between the vector fields (and hence charges) and the elements of the Jordan algebra extends to a four-dimensional correspondence between the field strengths (and their magnetic duals) and the elements of Freudenthal triple system defined over the corresponding Jordan algebra [37, 4, 7]. The automorphism groups of the FTSs are isomorphic to the $U$-duality groups of the corresponding four-dimensional $N=2$ MESGTs. Using the action of the automorphism group on the considered FTSs, we determine the orbits with non-vanishing quartic norms, which correspond to the quartic invariants of the MESGTs. We find three classes of such non-degenerate charge orbits, two with positive norm and one with a negative norm ${ }^{3}$.

Thence, the first two Subsects. of Sect. 4 are devoted to the general analysis of

\footnotetext{
${ }^{3}$ This is to be contrasted with the symmetric situation in $d=5$, where one finds two orbits with positive cubic norm and two with negative norm, which are pairwise isomorphic [1, 27].
} 
the three classes of regular extremal black hole attractors of $N=2, d=4$ symmetric "magical" MESGTs, and of the corresponding classes of non-degenerate charge orbits in the symplectic representation space of the relevant $d=4$ duality group. In particular, the $\frac{1}{2}$-BPS solutions are treated in Subsect. 4.1, while the two general species of non-BPS $Z \neq 0$ and non-BPS $Z=0$ attractors are respectively considered in Subsubsects. 4.2.1] and 4.2 .2 ,

Subsect. 4.3 deal with the two noteworthy cases of $N=2, d=4$ symmetric "magical"

MESGTs based on the manifolds $\frac{E_{7(-25)}}{E_{6} \otimes U(1)}$ (Subsubsect. 4.3.2) and $\frac{S O^{*}(12)}{U(6)}$ (Subsubsect. 4.3.3). This latter homogeneous symmetric special Kähler manifold is considered also in Subsubsect. 4.3.1, where its dual role in the interplay between $N=2$ and $N=6, d=4$ SUGRAs is pointed out.

The splittings of the mass spectra of $N=2, d=4$ symmetric "magical" MESGTs along their three classes of non-degenerate charge orbits are studied in Sect. 5.

Finally, the concluding Sect. 6] contains some general remarks and observations, as well as an outlook of possible future further developments along the considered research directions.

Two Appendices conclude the paper; they treat the regular attractors, non-degenerate charge orbits and critical mass spectra of the $N=2, d=4$ symmetric MESGTs based on the sequences $\frac{S U(1,1+n)}{U(1) \otimes S U(1+n)}$ (Appendix I) and $\frac{S U(1,1)}{U(1)} \otimes \frac{S O(2,2+n)}{S O(2) \otimes S O(2+n)}$ (Appendix II).

\section{$2 \quad$ BPS and non-BPS Attractors : Charge Orbits of $N=2, d=4$ MESGTs}

The symmetric special Kähler manifolds of $N=2, d=4$ MESGTs have been classified in the literature [40, 41]. With the exception of the family whose prepotential is quadratic, all such theories can be obtained by dimensional reduction of the $N=2, d=5$ MESGTs that were constructed in [37, 38, 39]. The MESGTs with symmetric manifolds that originate from 5 dimensions all have cubic prepotentials determined by the norm form of the Jordan algebra of degree three that defines them [37, 38, 39]. They include the two infinite sequences

$$
\begin{aligned}
& I: \frac{S U(1,1+n)}{U(1) \otimes S U(1+n)}, r=1 ; \\
& I I: \frac{S U(1,1)}{U(1)} \otimes \frac{S O(2,2+n)}{S O(2) \otimes S O(2+n)}, r=3,
\end{aligned}
$$

where $r$ stands for the rank of the coset. Of these two infinite families, the first family is the one whose prepotentials are quadratic. The second family has a five dimensional origin and its associated Jordan algebras are not simple. It is referred to as the generic Jordan family since it exists for any $n$. The first elements of such sequences (obtained for 
$n=0)$ respectively correspond to the following manifolds and holomorphic prepotential functions in special coordinates:

$$
\begin{aligned}
& I_{0}: \frac{S U(1,1)}{U(1)}, F(t)=\frac{i}{4}\left(t^{2}-1\right) \\
& I I_{0}: \frac{S U(1,1) \otimes S O(2,2)}{U(1) \otimes S O(2) \otimes S O(2)}=\left(\frac{S U(1,1)}{U(1)}\right)^{3}, F(s, t, u)=s t u .
\end{aligned}
$$

In general, all manifolds of type $I$ correspond to quadratic prepotentials $\left(C_{i j k}=0\right)$, as well as all manifolds of type $I I$ correspond to cubic prepotentials (in special coordinates $F=\frac{1}{3 !} d_{i j k} t^{i} t^{j} t^{k}$ and therefore $C_{i j k}=e^{K} d_{i j k}$, where $K$ denotes the Kähler potential and $d_{i j k}$ is a completely symmetric rank-3 constant tensor). The 3 -moduli case $I I_{0}$ is the well-known stu model [42, whose noteworthy triality symmetry has been recently related to quantum information theory [43, 44, 45].

Beside the infinite sequence $I I$, there exist four other MESGTs defined by simple Jordan algebras of degree three with the following rank-3 coset manifolds:

$$
\begin{aligned}
& I I I: \frac{E_{7(-25)}}{E_{6} \otimes U(1)} ; \\
& I V: \frac{S O^{*}(12)}{U(6)} ; \\
& V: \frac{S U(3,3)}{S(U(3) \otimes U(3))}=\frac{S U(3,3)}{S U(3) \otimes S U(3) \otimes U(1)} ; \\
& V I: \frac{S p(6, \mathbb{R})}{U(3)} .
\end{aligned}
$$

The $N=2, d=4$ MESGTs whose geometry of scalar fields is given by the manifolds $I I I$ $V I$ are called "magical", since their symmetry groups are the groups of the famous Magic Square of Freudenthal, Rozenfeld and Tits associated with some remarkable geometries [46, 47]. The four $N=2, d=4$ "magical" MESGTs $I I I-V I$, as their 5-d. versions, are defined by four simple Jordan algebras $J_{3}^{\mathbb{D}}, J_{3}^{\mathbb{H}}, J_{3}^{\mathbb{C}}$ and $J_{3}^{\mathbb{R}}$ of degree 3 with irreducible norm forms, namely by the Jordan algebras of Hermitian $3 \times 3$ matrices over the four division algebras, i.e. respectively over the octonions $\mathbb{O}$, quaternions $\mathbb{H}$, complex numbers $\mathbb{C}$ and real numbers $\mathbb{R}$ [37, 38, 39, 48, 49, 150, 51].

By denoting with $n_{V}$ the number of vector multiplets coupled to the SUGRA one, the total number of Abelian vector fields in the considered $N=2, d=4$ MESGT is $n_{V}+1$; correspondingly, the real dimension of the corresponding scalar manifold is $2 n_{V}=\operatorname{dim}(G)-\operatorname{dim}\left(H_{0}\right)-1$. Since the $2\left(n_{V}+1\right)$-dim. vector of extremal black hole charge configuration is given by the fluxes of the electric and magnetic field-strength two-forms, it is clear that $\operatorname{dim}_{\mathbb{R}}\left(R_{V}\right)=2\left(n_{V}+1\right)$.

Since $H_{0}$ is a proper compact subgroup of the duality semisimple group $G$, we can decompose the $2\left(n_{V}+1\right)$-dim. real symplectic representation $R_{V}$ of $G$ in terms of complex 


\begin{tabular}{|c||c|c|}
\hline & $I$ & $I I$ \\
\hline \hline$G$ & $S U(1,1+n)$ & $S U(1,1) \otimes S O(2,2+n)$ \\
\hline$H_{0}$ & $S U(1+n)$ & $S O(2) \otimes S O(2+n)$ \\
\hline$r$ & 1 & 3 \\
\hline $\operatorname{dim}_{\mathbb{R}}\left(\frac{G}{H_{0} \otimes U(1)}\right)$ & $2(n+1)$ & $2(n+3)$ \\
\hline$n_{V}$ & $n+r=n+1$ & $n+r=n+3$ \\
\hline$R_{V}$ & $(\mathbf{2}(\mathbf{n}+\mathbf{2}))_{\mathbb{R}}$ & $(\mathbf{2}(\mathbf{n}+\mathbf{4}))_{\mathbb{R}}$ \\
\hline$R_{H_{0}}$ & $(\mathbf{n}+\mathbf{1})_{\mathbb{C}}$ & $(\mathbf{n}+\mathbf{2}+\mathbf{1})_{\mathbb{C}}$ \\
\hline $\operatorname{dim}_{\mathbb{R}}\left(R_{V}\right)$ & $2(n+2)$ & $2(n+4)$ \\
\hline $\operatorname{dim}_{\mathbb{R}}\left(R_{H_{0}}\right)$ & $2(n+1)$ & $2(n+3)$ \\
\hline$R_{V}$ & $(\mathbf{2}(\mathbf{n}+\mathbf{2}))_{\mathbb{R}}$ & $(\mathbf{2}(\mathbf{n}+\mathbf{4}))_{\mathbb{R}}$ \\
$\downarrow$ & $\downarrow$ & $\downarrow$ \\
$R_{H_{0}}+\mathbf{1}_{\mathbb{C}}+$ & $(\mathbf{n}+\mathbf{1})_{\mathbb{C}}+\mathbf{1}_{\mathbb{C}}+$ & $(\mathbf{n}+\mathbf{2}+\mathbf{1})_{\mathbb{C}}+\mathbf{1}_{\mathbb{C}}+$ \\
+ c.c. & + c.c. & + c.c. \\
\hline
\end{tabular}

Table 1: Basic data of the two sequences of symmetric $N=2, d=4$ MESGTs

representations of $H_{0}$, obtaining in general the following decomposition scheme:

$$
R_{V} \longrightarrow R_{H_{0}}+\overline{R_{H_{0}}}+\mathbf{1}_{\mathbb{C}}+\overline{\mathbf{1}}_{\mathbb{C}}=R_{H_{0}}+\mathbf{1}_{\mathbb{C}}+\text { c.c. }
$$

where "c.c." stands for the complex conjugation of representations, and $R_{H_{0}}$ is a certain complex representation of $H_{0} \cdot{ }^{4}$

The basic data of the cases $I-V I$ listed above are summarized in Tables 1 and 2 .

It was shown in [1] that $\frac{1}{2}$-BPS orbits of $N=2, d=4$ symmetric MESGTs are coset spaces of the form

$$
\begin{aligned}
& \mathcal{O}_{\frac{1}{2}-B P S}=\frac{G}{H_{0}} \\
& \operatorname{dim}_{\mathbb{R}}\left(\mathcal{O}_{\frac{1}{2}-B P S}\right)=\operatorname{dim}(G)-\operatorname{dim}\left(H_{0}\right)=2 n_{V}+1=\operatorname{dim}_{\mathbb{R}}\left(R_{V}\right)-1 .
\end{aligned}
$$

Now, in order to proceed further, we need to consider the $N=2$ extremal black attractor eqs.; these are nothing but the criticality conditions for the $N=2$ black hole scalar potential [52, 11$]$

$$
V_{B H} \equiv|Z|^{2}+G^{i \bar{i}} D_{i} Z \bar{D} \bar{i} \bar{Z}
$$

in the corresponding special Kähler geometry [14]:

$$
\partial_{i} V_{B H}=0 \Longleftrightarrow 2 \bar{Z} D_{i} Z+i C_{i j k} G^{j \bar{j}} G^{k \bar{k}} \bar{D} \bar{j} \bar{Z} \bar{D}_{\bar{k}} \bar{Z}=0, \forall i=1, \ldots, n_{V} .
$$

\footnotetext{
${ }^{4}$ As will be discussed in Sect. 3, this decomposition reflects the decomposition of the corresponding Freudenthal triple system with respect to the underlying Jordan algebra.
} 


\begin{tabular}{|c||c|c|c|c|}
\hline & $J_{3}^{\circledast} \leftrightarrow I I I$ & $J_{3}^{\mathbb{H}} \leftrightarrow I V$ & $J_{3}^{\mathbb{C}} \leftrightarrow V$ & $J_{3}^{\mathbb{R}} \leftrightarrow V I$ \\
\hline \hline$G$ & $E_{7(-25)}$ & $S O^{*}(12)$ & $S U(3,3)$ & $S p(6, \mathbb{R})$ \\
\hline$H_{0}$ & $E_{6}$ & $S U(6)$ & $S U(3) \otimes S U(3)$ & $S U(3)$ \\
\hline$r$ & 3 & 3 & 3 & 3 \\
\hline $\operatorname{dim}_{\mathbb{R}}\left(\frac{G}{H_{0} \otimes U(1)}\right)$ & 54 & 30 & 18 & 12 \\
\hline$n_{V}$ & 27 & 15 & 9 & 6 \\
\hline$R_{V}$ & $\mathbf{5 6}_{\mathbb{R}}$ & $\mathbf{3 2}_{\mathbb{R}}$ & $\mathbf{1 0}_{\mathbb{R}}$ & $\mathbf{1 4}_{\mathbb{R}}^{\prime}$ \\
\hline$R_{H_{0}}$ & $\mathbf{2 7}_{\mathbb{C}}$ & $\mathbf{1 5}_{\mathbb{C}}$ & $\left(\mathbf{3}^{\prime}\right)_{\mathbb{C}}$ & $\mathbf{6}_{\mathbb{C}}$ \\
\hline $\operatorname{dim}_{\mathbb{R}}\left(R_{V}\right)$ & 56 & 32 & 20 & 14 \\
\hline $\operatorname{dim}_{\mathbb{R}}\left(R_{H_{0}}\right)$ & 54 & 30 & 18 & 12 \\
\hline$R_{V}$ & $\mathbf{5 6 _ { \mathbb { R } }}$ & $\mathbf{3 2}_{\mathbb{R}}$ & $\mathbf{1 0}_{\mathbb{R}}$ & $\mathbf{1 4}_{\mathbb{R}}^{\prime}$ \\
$\downarrow$ & $\downarrow$ & $\downarrow$ & $\downarrow$ & $\downarrow$ \\
$R_{H_{0}}+\mathbf{1}_{\mathbb{C}}+$ & $\mathbf{2 7}_{\mathbb{C}}+\mathbf{1}_{\mathbb{C}}+$ & $\mathbf{1 5}_{\mathbb{C}}+\mathbf{1}_{\mathbb{C}}+$ & $\left(\mathbf{3}, \mathbf{3}^{\prime}\right)_{\mathbb{C}}+\mathbf{1}_{\mathbb{C}}+$ & $\mathbf{6}_{\mathbb{C}}+\mathbf{1}_{\mathbb{C}}+$ \\
+ c.c. & + c.c. & + c.c. & + c.c. & + c.c. \\
\hline
\end{tabular}

Table 2: Basic data of the four "magical" symmetric $N=2, d=4$ MESGTs. $\mathbf{1 4}_{\mathbb{R}}^{\prime}$ is the rank-3 antisymmetric tensor representation of $S p(6, \mathbb{R})$. In $\left(\mathbf{3}, \mathbf{3}^{\prime}\right)_{\mathbb{C}}$ the prime distinguishes the representations of the two distinct $S U(3)$ groups.

$C_{i j k}$ is the rank-3, completely symmetric, covariantly holomorphic tensor of special Kähler geometry, satisfying (see e.g. [53])

$$
\bar{D}_{\bar{l}} C_{i j k}=0, \quad D_{[l} C_{i] j k}=0,
$$

where the square brackets denote antisymmetrization with respect to the enclosed indices.

For symmetric special Kähler manifolds the tensor $C_{i j k}$ is covariantly constant:

$$
D_{i} C_{j k l}=0,
$$

which further implies [38, 40]

$$
G^{k \bar{k}} G^{r \bar{j}} C_{r(p q} C_{i j) k} \bar{C}_{\overline{k i j}}=\frac{4}{3} G_{(q \mid \bar{i}} C_{\mid i j p)} .
$$

This equation is simply the four dimensional version of the adjoint identity satisfied by all Jordan algebras of degree three that define the corresponding MESGTs in five dimensions.

$Z$ is the $N=2$ "central charge" function, whereas $\left\{D_{i} Z\right\}_{i=1, \ldots, n_{V}}$ is the set of its Kähler-covariant holomorphic derivatives, which are nothing but the "matter charges" functions of the system. Indeed, the $\operatorname{sets}^{5}\left\{q_{0}, q_{i}, p^{0}, p^{i}\right\} \in \mathbb{R}^{2 n_{V}+2}$ and $\left\{Z, D_{i} Z\right\} \in \mathbb{C}^{n_{V}+1}$ (when evaluated at purely $(q, p)$-dependent critical values of the moduli) are two equivalent basis for the charges of the system, and they are related by a particular set of

\footnotetext{
${ }^{5}$ We always consider "classical" frameworks, disregarding the actual quantization of the ranges of the electric and magnetic charges $q_{0}, q_{i}, p^{0}$ and $p^{i}$. That is why we consider $\mathbb{R}^{2 n_{V}+2}$ rather than the $\left(2 n_{V}+2\right)$-dim. charge lattice $\widehat{\Gamma}_{(p, q)}$.
} 
identities of special Kähler geometry [52, 54, 55]. The decomposition (2.9) corresponds to nothing but the splitting of the sets $\left\{q_{0}, q_{i}, p^{0}, p^{i}\right\}\left(\left\{Z, D_{i} Z\right\}\right)$ of $2 n_{V}+2\left(n_{V}+1\right)$ real (complex) charges ("charge" functions) in $q_{0}, p^{0}(Z)$ (related to the graviphoton, and corresponding to $\mathbf{1}_{\mathbb{C}}+$ c.c.) and in $\left\{q_{i}, p^{i}\right\}\left(\left\{D_{i} Z\right\}\right)$ (related to the $n_{V}$ vector multiplets, and corresponding to $R_{H_{0}}+$ c.c.).

In order to perform the subsequent analysis of orbits, it is convenient to use "flat" $I$-indices by using the (inverse) $n_{V}$-bein $e_{I}^{i}$ of $\frac{G}{H_{0} \otimes U(1)}$ :

$$
D_{I} Z=e_{I}^{i} D_{i} Z
$$

By switching to "flat" local $I$-indices, the special Kähler metric $G_{i \bar{j}}$ (assumed to be regular, i.e. strictly positive definite everywhere) will become nothing but the Euclidean $n_{V}$-dim. metric $\delta_{I \bar{J}}$. Thus, the attractor eqs. (2.12) can be "flatted" as follows:

$$
\partial_{I} V_{B H}=0 \Longleftrightarrow 2 \bar{Z} D_{I} Z+i C_{I J K} \delta^{J \bar{J}} \delta^{K \bar{K}} \bar{D} \bar{J}_{\bar{Z}} \bar{D} \bar{K} \bar{Z}=0, \forall I=1, \ldots, n_{V}
$$

Note that $C_{I J K}$ becomes an $H_{0}$-invariant tensor [56]. This is possible because $C_{i j k}$ in special coordinates is proportional to the invariant tensor $d_{I J K}$ of the $d=5 U$-duality group $G_{5} . G_{5}$ and $H_{0}$ correspond to two different real forms of the same Lie algebra [38].

As it is well known, $\frac{1}{2}$-BPS attractors are given by the following solution [14] of attractor eqs. (2.12) and (2.17):

$$
Z \neq 0, D_{i} Z=0 \Leftrightarrow D_{I} Z=0, \forall i, I=1, \ldots, n_{V}
$$

Since the "flatted matter charges" $D_{I} Z$ are a vector of $R_{H_{0}}$, Eq. (2.18) directly yields that $\frac{1}{2}$-BPS solutions are manifestly $H_{0}$-invariant. In other words, since the $N=2$, $\frac{1}{2}$-BPS orbits are of the form $\frac{G}{H_{0}}$, the condition for the $\left(n_{V}+1\right)$-dim. complex vector $\left(Z, D_{i} Z\right)$ to be $H_{0}$-invariant is precisely given by Eq. (2.18), defining $N=2$, $\frac{1}{2}$-BPS attractor solutions.

Thus, as for the $N=8$ regular solutions (1.6) and (1.8), also for the $N=2 \frac{1}{2}$-BPS case the invariance properties of the solutions at the critical point(s) are given by the m.c.s. of the stabilizer of the corresponding charge orbit, which in the present case is the compact stabilizer itself. Thus, at $N=2 \frac{1}{2}$-BPS critical points the following enhancement of symmetry holds:

$$
\mathcal{S} \longrightarrow H_{0}
$$

where here and below $\mathcal{S}$ denotes the compact symmetry of a generic orbit of the real symplectic representation $R_{V}$ of the $d=4$ duality group $G$.

However, all the scalar manifolds of $N=2, d=4$ symmetric MESGTs have other species of regular critical points $V_{B H}$ (and correspondingly other classes of non-degenerate charge orbits). 
In recent months ( $N=2$ non-BPS) non-supersymmetric extremal black hole attractors have been studied in the literature [15]-[25], also in the case of non-homogeneous (and non-symmetric) special Kähler manifolds, e.g. in the large volume limit of Calabi-Yau compactifications of Type IIA superstrings [17]. Away from such a limit, exact non-BPS solutions have been given for $n_{V}=1$ in the case of quintic in $\mathbb{C} P^{4}$ (working in the IIB mirror description) and of sixtic in $W P_{1,1,1,1,2}^{4}$ respectively in [17] and [18. While all treated cases are $N=2$ non-BPS, $Z \neq 0$ attractors, in [18 the first (and, as far as we know, the only) known example of $N=2$ non-BPS, $Z=0$ attractor is presented.

Concerning the $N=2, d=4$ symmetric MESGTs, the rank-1 sequence $I$ has one more, non-BPS class of orbits (with vanishing central charge), while all rank-3 aforementioned cases $I I-V I$ have two more distinct non-BPS classes of orbits, one of which with vanishing central charge.

The results about the classes of non-degenerate charge orbits of $N=2, d=4$ symmetric MESGTs are summarized in Table $3^{6}$.

\section{Freudenthal Triple Systems and$$
\text { Orbits of Symmetric } N=2, d=4 \text { MESGTs }
$$

In those symmetric $N=2, d=5$ MESGTs whose cubic norm form is taken to be the norm form of an Euclidean Jordan algebra $\mathcal{J}$ of degree three, there is an one-to-one correspondence between the vector fields (and hence their charges) and the elements of $\mathcal{J}$ 37, 38, 39].

Under dimensional reduction to $d=4$, such a correspondence gets extended to a correspondence between the field strengths of the vector fields (and their magnetic duals) and the Freudenthal triple system (FTS) [57, 46] $\mathcal{F}(\mathcal{J})$ defined over $\mathcal{J}$ [37, 3, 1, 4, 7], and it can be realized as $2 \times 2$ "matrices" :

$$
\left(\begin{array}{cc}
F_{\mu \nu}^{0} & F_{\mu \nu}^{i} \\
\widetilde{F}_{i}^{\mu \nu} & \widetilde{F}_{0}^{\mu \nu}
\end{array}\right) \Longleftrightarrow\left(\begin{array}{cc}
\alpha & x \\
y & \beta
\end{array}\right) \in \mathcal{F}(\mathcal{J}),
$$

\footnotetext{
${ }^{6}$ It is here worth remarking that the column on the right of Table 2 of [1] is not fully correct.

Indeed, such a column coincides with the central column of Table 3 of the present paper (by disregarding case $I$ and shifting $n \rightarrow n-2$ in case $I I$ ), listing the non-BPS, $Z \neq 0$ orbits of $N=2, d=4$ symmetric MESGTs, which are all characterized by a strictly negative quartic $E_{7}$-invariant $I_{4}$. This does not match what is claimed in [1, where such a column is stated to list the particular class of orbits with $I_{4}>0$ and eigenvalues of opposite sign in pair.

Actually, the statement of [1] holds true only for the case $I$ (which, by shifting $n \rightarrow n-1$, coincides with the last entry of the column on the right of Table 2 of [1]). On the other hand, such a case is the only one which cannot be obtained from $d=5$ by dimensional reduction. Moreover, it is the only one not having non-BPS, $Z \neq 0$ orbits, rather it is characterized only by a class of non-BPS orbits with $Z=0$ and $I_{4}>0$.
} 


\begin{tabular}{|c|c|c|c|}
\hline & $\begin{array}{l}\frac{1}{2} \text {-BPS orbits } \\
\mathcal{O}_{\frac{1}{2}-B P S}=\frac{G}{H_{0}}\end{array}$ & $\begin{array}{c}\text { non-BPS, } Z \neq 0 \text { orbits } \\
\mathcal{O}_{\text {non-BPS,Z } \neq 0}=\frac{G}{\widehat{H}}\end{array}$ & $\begin{array}{c}\text { non-BPS, } Z=0 \text { orbits } \\
\mathcal{O}_{\text {non }-B P S, Z=0}=\frac{G}{H}\end{array}$ \\
\hline$I$ & $\frac{S U(1, n+1)}{S U(n+1)}$ & - & $\frac{S U(1, n+1)}{S U(1, n)}$ \\
\hline$I I$ & $\frac{S U(1,1) \otimes S O(2,2+n)}{S O(2) \otimes S O(2+n)}$ & $\frac{S U(1,1) \otimes S O(2,2+n)}{S O(1,1) \otimes S O(1,1+n)}$ & $\frac{S U(1,1) \otimes S O(2,2+n)}{S O(2) \otimes S O(2, n)}$ \\
\hline$I I I$ & $\frac{E_{7(-25)}}{E_{6}}$ & $\frac{E_{7(-25)}}{E_{6(-26)}}$ & $\frac{E_{7(-25)}}{E_{6(-14)}}$ \\
\hline$I V$ & $\frac{S O^{*}(12)}{S U(6)}$ & $\frac{S O^{*}(12)}{S U^{*}(6)}$ & $\frac{S O^{*}(12)}{S U(4,2)}$ \\
\hline$V$ & $\frac{S U(3,3)}{S U(3) \otimes S U(3)}$ & $\frac{S U(3,3)}{S L(3, \mathbb{C})}$ & $\frac{S U(3,3)}{S U(2,1) \otimes S U(1,2)}$ \\
\hline$V I$ & $\frac{S p(6, \mathbb{R})}{S U(3)}$ & $\frac{S p(6, \mathbb{R})}{S L(3, \mathbb{R})}$ & $\frac{S p(6, \mathbb{R})}{S U(2,1)}$ \\
\hline
\end{tabular}

Table 3: Non-degenerate orbits of $N=2, d=4$ symmetric MESGTs

where $\alpha, \beta \in \mathbb{R}$ and $x, y \in \mathcal{J} . F_{\mu \nu}^{0}$ is the $d=4$ graviphoton field strength, i.e. the field strength of the vector field coming from the $d=5$ graviton; $F_{\mu \nu}^{i}$ denote the field strengths of the vector fields that already exist in $d=5$ (notice that $i=1, \ldots, \widehat{n}+1=n_{V}$, where $n_{V}$, $\widehat{n}$ respectively stand for the number of Abelian vector multiplets in $d=4,5$ dimensions) [37, 38, 39].

Consequently, one can associate an element of the FTS $\mathcal{F}(\mathcal{J})$ with the electric and magnetic charges $\left\{q_{0}, q_{i}, p^{0}, p^{i}\right\} \in \mathbb{R}^{2 n_{V}+2}$ (fluxes of the corresponding field strengths) of an $N=2, d=4$ (extremal) black hole:

$$
\left(\begin{array}{cc}
p^{0} & p^{i} \\
q_{i} & q_{0}
\end{array}\right) \Longleftrightarrow\left(\begin{array}{cc}
\alpha & x \\
y & \beta
\end{array}\right) \in \mathcal{F}(\mathcal{J})
$$

where $\alpha=p^{0}, \beta=q_{0}, x=p^{i} j_{i}$ and $y=q_{i} j^{i}$, with $\left\{j_{i}\right\}_{i=1, \ldots, n_{V}}$ denoting the set of basis vectors of $\mathcal{J}$.

In general, an FTS is defined as a vector space $\mathcal{M}$ with a trilinear product $(P, Q, R)$ and a skew-symmetric bilinear form $\langle P, Q\rangle, P, Q, R \in \mathcal{M}$. One can always modify the triple product $(P, Q, R)$ by adding terms like $\langle P, Q\rangle R$ in order to make it completely 
symmetric. In our treatment we shall assume the triple product to be completely symmetric, and we will follow the conventions of Brown [58], who axiomatized the triple systems introduced by Freudenthal in his study of the exceptional groups of the Magic Square [57, 46].

As reformulated by Ferrar [59], an FTS is a vector space $\mathcal{M}$ endowed with a trilinear product $(P, Q, R)$ and a skew-symmetric bilinear form $\langle P, Q\rangle=-\langle Q, P\rangle$, such that $(\forall P, Q, R, S \in \mathcal{M})$ :

A1. $(P, Q, R)$ is completely symmetric;

A2.

$$
\mathcal{Q}(P, Q, R, S) \equiv\langle P,(Q, R, S)\rangle
$$

is a non-zero completely symmetric 4-linear form;

A3.

$$
((P, P, P), P, Q)=\langle Q, P\rangle(P, P, P)+\langle Q,(P, P, P)\rangle P
$$

For FTSs defined over an (Euclidean) Jordan algebra $\mathcal{J}$ of degree three, it is customary to denote the elements of $\mathcal{M}$ as follows:

$$
\begin{aligned}
& \mathcal{M} \ni P=\left(\begin{array}{cc}
\alpha & x \\
y & \beta
\end{array}\right) \equiv(\alpha, \beta, x, y) ; \\
& \mathcal{M} \ni Q=\left(\begin{array}{cc}
\gamma & w \\
z & \delta
\end{array}\right) \equiv(\gamma, \delta, w, z),
\end{aligned}
$$

where $\alpha, \beta, \gamma, \delta \in \mathbb{R}$ and $x, y, w, z \in \mathcal{J}$.

Thence

$$
\langle P, Q\rangle \equiv \alpha \delta-\beta \gamma+T(x, z)-T(y, w),
$$

where $T(x, z) \equiv \operatorname{Tr}(x \circ z)$, with " $T r$ " denoting the trace of the $(3 \times 3$ matrix repre-

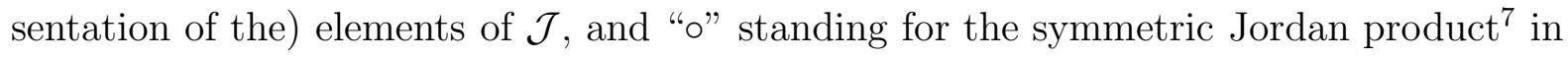
$\mathcal{J}$.

The quartic norm of an element $P \in \mathcal{M}$ is defined as

$$
I_{4}(P) \equiv \mathcal{Q}(P, P, P, P),
$$

\footnotetext{
${ }^{7}$ It should be mentioned that the symmetric Jordan product $\circ$ satisfies the famous Jordan identity 48, 49, 50, 51, 37, 38, 39] ( $\forall x, y$ elements of the Jordan algebra)

$$
x \circ\left(y \circ x^{2}\right)=(x \circ y) \circ x^{2} .
$$
}


and it can be normalized such that ${ }^{8}$ 60

$$
I_{4}(P)=-\{\alpha \beta-T(x, z)\}^{2}-4\left\{\alpha I_{3}(y)+\beta I_{3}(x)-T\left(x^{\#}, y^{\#}\right)\right\}
$$

where $I_{3}$ denotes the cubic norm of $\mathcal{J}$ and "\#" stands for the quadratic adjoint map of $\mathcal{J}$. By introducing the Freudenthal cross (symmetric) product

$$
x \wedge y \equiv x \circ y-\frac{1}{2}[\operatorname{Tr}(x) y+\operatorname{Tr}(y) x]+\frac{1}{2}[\operatorname{Tr}(x) \operatorname{Tr}(y)-\operatorname{Tr}(x \circ y)],
$$

the quadratic adjoint map of $x \in \mathcal{J}$ can be defined as

$$
x^{\#} \equiv x \wedge x
$$

and it can be shown that it has the iteration property 61

$$
x^{\# \#}=I_{3}(x) x .
$$

The automorphism group $A u t(\mathcal{F}(\mathcal{J}))$ of the FTS defined over an Euclidan Jordan algebra $\mathcal{J}$ of degree three contains as proper subgroup the structure group $\operatorname{Str}(\mathcal{J})$ of $\mathcal{J}$, i.e. the group which leaves the cubic norm $I_{3}$ of $\mathcal{J}$ invariant up to a real overall scale factor $\lambda$. Furthermore, those transformations belonging to $\operatorname{Str}(\mathcal{J})$ that leave the cubic norm $I_{3}$ invariant (i.e. such that $\lambda=1$ ) form the reduced structure group [49] $\operatorname{Str}_{0}(\mathcal{J})$ of $\mathcal{J}$, which is nothing but the $U$-duality group $G_{5}$ of the corresponding $N=2, d=5$ symmetric MESGT. Indeed, the symmetric $N=2, d=5$ MESGTs whose cubic norm form is taken to be the norm form of an Euclidean Jordan algebra $\mathcal{J}$ of degree three are endowed with homogeneous symmetric real ${ }^{9}$ manifolds of the form

$$
\frac{G_{5}}{H_{5}}=\frac{\operatorname{Str}_{0}(\mathcal{J})}{\operatorname{Aut}(\mathcal{J})}
$$

where $\operatorname{Aut}(\mathcal{J})=$ m.c.s. $\left(\operatorname{Str}_{0}(\mathcal{J})\right)$ denotes the automorphism group of $\mathcal{J}$. For further elucidation on the $\mathcal{J}$-related symmetric $N=2, d=5$ MESGTs, see [37, 38, 39, 62] and [1, 27.

With the exception of the family with the scalar manifold $\frac{S U(1,1+n)}{U(1) \otimes S U(1+n)}$, all symmetric $N=2, d=4$ MESGTs can be obtained from the corresponding $d=5$ theories by dimensional reduction [37, 38, 39, 62]. The automorphism group $\operatorname{Aut}(\mathcal{F}(\mathcal{J}))$ of $\mathcal{F}(\mathcal{J})$

\footnotetext{
${ }^{8}$ Eq. (3.8) corrects a misprint in Eq. (2-15) of [1, by substituting the factor 6 with 4 and introducing an overall sign.

${ }^{9}$ Sometimes in the literature the geometry of such manifolds is referred to as real special geometry, whereas the geometry of the manifolds of the corresponding $d=4$ MESGTs obtained by dimensional reduction is sometimes named very special Kähler geometry.
} 
is nothing but the $U$-duality group $G \equiv G_{4}$ of the $\mathcal{J}$-related MESGT in $d=4^{10}$ :

$$
\begin{aligned}
& I I: \operatorname{Aut}\left(\mathcal{F}\left(\mathbb{R} \oplus \boldsymbol{\Gamma}_{n+2}\right)\right)=S U(1,1) \otimes S O(2,2+n) ; \\
& I I I: \operatorname{Aut}\left(\mathcal{F}\left(J_{3}^{\mathbb{Q}}\right)\right)=E_{7(-25)} ; \\
& I V: \operatorname{Aut}\left(\mathcal{F}\left(J_{3}^{\mathbb{H}}\right)\right)=S O^{*}(12) \\
& V: \operatorname{Aut}\left(\mathcal{F}\left(J_{3}^{\mathbb{C}}\right)\right)=S U(3,3) ; \\
& V I: \operatorname{Aut}\left(\mathcal{F}\left(J_{3}^{\mathbb{R}}\right)\right)=S p(6, \mathbb{R}) .
\end{aligned}
$$

Thus, the homogeneous symmetric special Kähler scalar manifold of $\mathcal{J}$-related symmetric $N=2, d=4$ MESGTs can be generally written as

$$
\frac{G_{4}}{H_{4}}=\frac{\operatorname{Aut}(\mathcal{F}(\mathcal{J}))}{\text { m.c.s. }(A u t(\mathcal{F}(\mathcal{J})))}=\frac{\operatorname{Aut}(\mathcal{F}(\mathcal{J}))}{H_{0} \otimes U(1)}
$$

and the following chain of strict group inclusions hold:

$$
H_{5}=\operatorname{Aut}(\mathcal{J})=\text { m.c.s. }\left(G_{5}\right) \varsubsetneqq G_{5}=\operatorname{Str}_{0}(\mathcal{J}) \varsubsetneqq \operatorname{Str}(\mathcal{J}) \varsubsetneqq \operatorname{Aut}(\mathcal{F}(\mathcal{J}))=G \equiv G_{4} .
$$

The global action of $A u t(\mathcal{F}(\mathcal{J}))$ on $\mathcal{F}(\mathcal{J})$ is generated by the structure group $\operatorname{Str}(\mathcal{J})$ of $\mathcal{J} S_{\lambda}(\cdot)(\lambda \in \mathbb{R})$, by a discrete transformation $\tau$ and by two additional $\mathcal{J}$-parameterized transformations $\phi(C)$ and $\psi(D)$. The $\lambda$-parameterized action of $\operatorname{Str}(\mathcal{J})$ reads

$$
\operatorname{Str}(\mathcal{J}):(\alpha, \beta, x, y) \longmapsto\left(\frac{\alpha}{\lambda}, \lambda \beta, S_{\lambda}(x), \widetilde{S}_{\lambda}(y)\right)
$$

where $S_{\lambda}$ and and $\tilde{S}_{\lambda}$ respectively denote the action of $\operatorname{Str}(\mathcal{J})$ on $\mathcal{J}$ and its adjoint. The transformation $\tau$ acts as

$$
\tau:(\alpha, \beta, x, y) \longmapsto(-\beta, \alpha,-y, x) .
$$

The additional transformations (parameterized by elements of $\mathcal{J}$ ) act as :

$$
\begin{aligned}
& \phi(C):(\alpha, \beta, x, y) \longmapsto\left(\alpha^{\prime}, \beta, x^{\prime}, y^{\prime}\right) \\
& \left\{\begin{array}{l}
\alpha^{\prime} \equiv \alpha+\beta I_{3}(C)+T(x, C \wedge C)+T(y, C) ; \\
x^{\prime} \equiv x+\beta C ; \\
y^{\prime} \equiv y+2 x \wedge C+\beta C \wedge C ; \\
C \in \mathcal{J}
\end{array}\right.
\end{aligned}
$$

\footnotetext{
${ }^{10} \mathbb{R} \oplus \boldsymbol{\Gamma}_{n+2}$ denotes the generic family of reducible Euclidean Jordan algebras of degree 3 . $\mathbb{R}$ denotes the one dimensional Jordan algebra and $\boldsymbol{\Gamma}_{n+2}$ denotes the Jordan algebra of degree 2 associated with a quadratic form of Lorentzian signature. This signature is required for the Jordan algebra $\mathcal{J}$ to be Euclidean, which is defined as a Jordan algebra in which the equation $x^{2}+y^{2}=0$ implies that $x=y=0$ for all elements $x, y$ of $\mathcal{J}$.
} 


$$
\begin{aligned}
& \psi(D):(\alpha, \beta, x, y) \longmapsto\left(\alpha, \beta^{\prime \prime}, x^{\prime \prime}, y^{\prime \prime}\right), \\
& \left\{\begin{array}{l}
\beta^{\prime \prime} \equiv \beta+\alpha I_{3}(D)+T(y, D \wedge D)+T(x, D) ; \\
x^{\prime \prime} \equiv x+\alpha D \wedge D+2 y \wedge D ; \\
y^{\prime \prime} \equiv y+\alpha D ; \\
D \in \mathcal{J} .
\end{array}\right.
\end{aligned}
$$

We should note that [49, 63]

$$
I_{3}(S(x))=\lambda I_{3}(x)
$$

and

$$
\widetilde{S}(x \wedge y)=S(x) \wedge S(y)
$$

Furthermore, $\operatorname{Str}(\mathcal{J})$ can be written as the direct product of the reduced structure group $\operatorname{Str}_{0}(\mathcal{J})$ times the dilatation group $\mathcal{D}$ :

$$
\operatorname{Str}(\mathcal{J})=\operatorname{Str}_{0}(\mathcal{J}) \otimes \mathcal{D} .
$$

Now, as it was shown by Krutelevich 64, by suitably acting with $\operatorname{Aut}(\mathcal{F}(\mathcal{J})$ ) one can bring a generic element of $\mathcal{F}(\mathcal{J})$ to the noteworthy form

$$
(1, \beta, x, 0) \in \mathcal{F}(\mathcal{J}), \beta \in \mathbb{R}, x \in \mathcal{J}
$$

Note that $\operatorname{Str}_{0}(\mathcal{J})$ preserves such a simplified form; indeed, Eq. (3.16) with $\lambda=1$ yields

$$
\operatorname{Str}_{0}(\mathcal{J}):(1, \beta, x, 0) \longmapsto\left(1, \beta, S_{0}(x), 0\right) .
$$

Moreover, by suitably acting with $\operatorname{Aut}(\mathcal{J})=H_{5}$, one can bring a generic element $x \in \mathcal{J}$ to the diagonal form:

$$
x \stackrel{\operatorname{Aut}(\mathcal{J})}{\longmapsto} \lambda_{1} E_{1}+\lambda_{2} E_{2}+\lambda_{3} E_{3}
$$

where $\lambda_{1}, \lambda_{2}, \lambda_{3} \in \mathbb{R}$ are the real "eigenvalues"11 of $x$ and $E_{1}, E_{2}, E_{3}$ are the three irreducible idempotents of $\mathcal{J}$ (see further below).

Thus, by using Eqs. (3.23) and (3.25) and by acting with the transformation $\phi\left(C=\varepsilon E_{1}\right)$ followed by the transformation $\psi\left(D=-\varepsilon\left(\lambda_{3} E_{2}+\lambda_{2} E_{3}\right)\right)$ (recall definitions (3.18) and (3.19) ), it can be shown that a generic element of $\mathcal{F}(\mathcal{J})$ can be brought to the form 64

$$
\begin{aligned}
& \left(1, \beta-2 \lambda_{2} \lambda_{3} \varepsilon, \lambda_{1}^{\prime} E_{1}+\lambda_{2} E_{2}+\lambda_{3} E_{3}, 0\right) \in \mathcal{F}(\mathcal{J}), \\
& \lambda_{1}^{\prime} \equiv-\lambda_{2} \lambda_{3} \varepsilon^{2}+\beta \varepsilon+\lambda_{1} .
\end{aligned}
$$

\footnotetext{
${ }^{11}$ For the simple Euclidean Jordan algebras of degree three they are the usual eigenvalues of the $3 \times 3$ Hermitian matrix representing $x$, while for the generic Jordan family $\mathbb{R}+\boldsymbol{\Gamma}_{n+2}$ they correspond to the values when a given element $x$ is brought to the form (3.25).
} 
Consequently, by choosing

$$
\mathbb{R}_{0} \ni \varepsilon: 2 \lambda_{2} \lambda_{3} \varepsilon=\beta,
$$

one can further simplify the form (3.26) into

$$
\begin{aligned}
& \left(1,0, \lambda_{1}^{\prime \prime} E_{1}+\lambda_{2} E_{2}+\lambda_{3} E_{3}, 0\right) \in \mathcal{F}(\mathcal{J}), \\
& \lambda_{1}^{\prime \prime} \equiv \lambda_{2} \lambda_{3} \varepsilon^{2}+\lambda_{1} .
\end{aligned}
$$

Eq. (3.28) shows that the sign of $\lambda_{1}^{\prime \prime} \in \mathbb{R}$ might differ from the sign of $\lambda_{1}$, depending on the sign of $\lambda_{2} \lambda_{3}$ and on the relative magnitude of $\lambda_{1}$ versus $\lambda_{2} \lambda_{3} \varepsilon^{2}$. In other words, the sign of the "eigenvalues" of the elements of $\mathcal{J}$ in the FTS $\mathcal{F}(\mathcal{J})$ could be changed by the action of $\operatorname{Aut}(\mathcal{F}(\mathcal{J}))$.

Now, by a suitable action of $\operatorname{Str}_{0}(\mathcal{J})$ one can bring a generic element $x \in \mathcal{J}$ to the diagonal form $\left(\nu \equiv \lambda_{1} \lambda_{2} \lambda_{3}\right)[27]$

$$
x_{\text {diag, } 1 \text { st }}=E_{1}+E_{2}+\nu E_{3}
$$

if at least two "eigenvalues" of $x$ are positive, or to the diagonal form

$$
x_{\text {diag, } 2 n d}=-E_{1}-E_{2}+\nu E_{3}
$$

if at least two "eigenvalues" of $x$ are negative (clearly, up to permutations of the indices $1,2,3)$.

For the case of $\mathcal{F}\left(J_{3}^{\mathbb{Q}}\right)$, it has been shown in [65] that for $\nu>0$ the element

$$
\left(1,0, E_{1}+E_{2}+\nu E_{3}, 0\right) \in \mathcal{F}\left(J_{3}^{\mathbb{Q}}\right)
$$

(having all three "eigenvalues" > 0) can be transformed into the element

$$
\left(1,0,-E_{1}-E_{2}+\nu E_{3}, 0\right) \in \mathcal{F}\left(J_{3}^{\mathbb{Q}}\right)
$$

(having two "eigenvalues" $<0$ and one "eigenvalue" $>0$ ) - and viceversa - by a suitable action of $A u t\left(\mathcal{F}\left(J_{3}^{\mathbb{\Phi}}\right)\right)=E_{7(-25)}$ (recall Eq. (3.13) $)$; instead, for $\nu<0$ it was proven that such two elements of $\mathcal{F}\left(J_{3}^{\mathbb{Q}}\right)$ cannot be transformed one into each other by the action of $E_{7(-25)}$ :

$$
\begin{aligned}
& \nu>0:\left(1,0, E_{1}+E_{2}+|\nu| E_{3}, 0\right) \stackrel{E_{7(-25)}}{\longleftrightarrow}\left(1,0,-E_{1}-E_{2}+|\nu| E_{3}, 0\right) ; \\
& \nu<0:\left(1,0, E_{1}+E_{2}-|\nu| E_{3}, 0\right) \stackrel{E_{7(-25)}}{\leftrightarrow}\left(1,0,-E_{1}-E_{2}-|\nu| E_{3}, 0\right) .
\end{aligned}
$$

Since the proof of [65] involves the idempotent elements $E_{1}, E_{2}, E_{3} \in \mathcal{J}$, it is easy to show that such a proof actually holds true for all $\mathcal{F}(\mathcal{J})$, where $\mathcal{J}$ is a Euclidean Jordan algebra of degree three. 
We shall use $\operatorname{Str}_{0}(\mathcal{J}) \varsubsetneqq A u t(\mathcal{F}(\mathcal{J})$ ) to determine the stabilizer (also called little group or isotropy group) of the various classes of charge orbits. Starting from a generic element of $\mathcal{F}(\mathcal{J})$ with at least two positive "eigenvalues" and using $\operatorname{Str}_{0}(\mathcal{J})$, one can recast it in the form

$$
\left(1,0,|\nu|^{1 / 3}\left(E_{1}+E_{2}+\operatorname{sgn}(\nu) E_{3}\right), 0\right)
$$

which in turn, by applying the scale transformation $\tau$, can be shown to become

$$
\kappa\left(1,0,\left(E_{1}+E_{2}+\operatorname{sgn}(\nu) E_{3}\right), 0\right)
$$

where $\kappa \equiv|\nu|^{1 / 4} \in \mathbb{R}_{0}^{+}$. On the other hand, when at least two of the "eigenvalues" are negative one can bring the considered element of $\mathcal{F}(\mathcal{J})$ to the form

$$
\left(1,0,|\nu|^{1 / 3}\left(-E_{1}-E_{2}+\operatorname{sgn}(\nu) E_{3}\right), 0\right)
$$

using $\operatorname{Str}_{0}(\mathcal{J})$, and then, through the scale transformation $\tau$, to the form

$$
\kappa\left(1,0,\left(-E_{1}-E_{2}+\operatorname{sgn}(\nu) E_{3}\right), 0\right) .
$$

We shall refer to the vector notations (3.35) and (3.37) of elements of $\mathcal{F}(\mathcal{J})$ as reference vectors.

In order to determine the little groups of the reference vectors belonging to each class of charge orbits, we shall use the action of the Lie algebra $\mathfrak{A} \mathfrak{u t}(\mathcal{F}(\mathcal{J}))$ of $\operatorname{Aut}(\mathcal{F}(\mathcal{J}))$. At the level of $\mathfrak{A} \mathfrak{u t}(\mathcal{F}(\mathcal{J})$ ), the generators of the little groups of the various classes of charge orbits annihilate the corresponding reference vectors. The subgroups of $\operatorname{Str}_{0}(\mathcal{J})$ that leave the above reference vectors invariant are known from [1, 27]. Therefore, in order to determine the stability group of a class of orbits under the action of $\operatorname{Aut}(\mathcal{F}(\mathcal{J})$ ), we need only to determine the generators outside the Lie algebra $\mathfrak{S t r}(\mathcal{J})$ of $\operatorname{Str}(\mathcal{J})$ (also called the structure algebra of $\mathcal{J}$ ) that annihilate the considered reference vector.

The group actions defined by Eqs. (3.18)-(3.19) can be shown to lead to the following actions by the elements of $\mathfrak{A} \mathfrak{u t}(\mathcal{F}(\mathcal{J})$ ) outside $\mathfrak{S} \mathfrak{t r}(\mathcal{J})$ (i.e. by the infinitesimal generators of $\operatorname{Aut}(\mathcal{F}(\mathcal{J}))$ which are not generators of $\operatorname{Str}(\mathcal{J}) \varsubsetneqq \operatorname{Aut}(\mathcal{F}(\mathcal{J}))$ ) [57, 46, 58, 66]:

$$
\begin{aligned}
& \mathcal{T}(A, B)(\alpha, \beta, x, y)=\left(\alpha^{\prime}, \beta^{\prime}, x^{\prime}, y^{\prime}\right) \\
& \left\{\begin{array}{l}
\alpha^{\prime} \equiv T(A, y) \\
\beta^{\prime} \equiv T(B, x) \\
x^{\prime} \equiv 2 B \wedge y+\beta A \\
y^{\prime} \equiv 2 A \wedge x+\alpha B \\
A, B \in \mathcal{J} .
\end{array}\right.
\end{aligned}
$$


The Hermitian conjugate of $\mathcal{T}(A, B)$ is defined as

$$
\mathcal{T}^{\dagger}(A, B) \equiv \mathcal{T}(B, A)
$$

and the non-compact generators are Hermitian, while the compact ones are anti-Hermitian.

We recall that for the simple Euclidean Jordan algebras of degree three $J_{3}^{\mathbb{A}}$ a generic element $A$ can be realized as a $3 \times 3$ Hermitian matrix over the underlying division algebras $\mathbb{A}=\mathbb{R}, \mathbb{C}, \mathbb{H}, \mathbb{O}$ as follows:

$$
A=\left(\begin{array}{ccc}
\alpha_{1} & a_{3} & a_{2}^{*} \\
a_{3}^{*} & \alpha_{2} & a_{1} \\
a_{3} & a_{1}^{*} & \alpha_{3}
\end{array}\right),
$$

where $\alpha_{i} \in \mathbb{R}, a_{i} \in \mathbb{A}(i=1,2,3)$, and "*" denotes conjugation in $\mathbb{A}$. The Jordan product o of two such elements $A$ and $B$ is simply

$$
A \circ B \equiv \frac{1}{2}(A \times B+B \times A)
$$

where " $\times$ " stands for the standard "row-column" matrix product. The irreducible idempotents of a Jordan algebra are those elements $E$ such that

$$
E^{2} \equiv E \circ E=E
$$

and

$$
\operatorname{Tr}(E)=1 \text {. }
$$

We shall denote the irreducible idempotents as $E_{i}(i=1,2,3)$, such that

$$
\operatorname{Tr}\left(A \circ E_{i}\right)=\alpha_{i}
$$

The cubic norm $I_{3}$ of a simple Euclidean Jordan algebra of degree three is simply the determinant of its $3 \times 3$ Hermitian matrix realization

$$
I_{3}(A) \equiv \alpha_{1} \alpha_{2} \alpha_{3}-\alpha_{1}\left|a_{1}\right|^{2}-\alpha_{2}\left|a_{2}\right|^{2}-\alpha_{3}\left|a_{3}\right|^{2}+2 R e\left(a_{1} a_{2} a_{3}\right),
$$

where $\operatorname{Re}(a) \equiv \frac{1}{2}\left(a+a^{*}\right)$ denotes the real part of $a \in \mathbb{A}$, and

$$
\operatorname{Re}\left(a_{1} a_{2} a_{3}\right)=\operatorname{Re}\left[a_{1}\left(a_{2} a_{3}\right)\right]=\operatorname{Re}\left[\left(a_{1} a_{2}\right) a_{3}\right]
$$

holds true for all elements of $\mathbb{A}$.

Concerning the generic reducible Jordan family $\mathcal{J}=\mathbb{R} \oplus \boldsymbol{\Gamma}_{n+2}(n \in \mathbb{N} \cup\{0\})$, a generic element $\mathcal{X}$ can be represented in the form

$$
\left(\zeta ; \xi_{0} \mathbf{1}+\vec{\xi} \cdot \vec{\sigma}\right)
$$


where $\zeta \in \mathbb{R}, \xi_{0} \in \mathbb{R}, \vec{\xi} \in \mathbb{R}^{n+1}$ and $\sigma_{i}$ are the gamma matrices in $\mathbb{R}^{n+1}$ :

$$
\left\{\sigma_{i}, \sigma_{j}\right\}=2 \delta_{i j}, i, j=1, \ldots, n+1 \text {. }
$$

The cubic norm of such an element is given by [37, 17]

$$
I_{3}(\mathcal{X}) \equiv \sqrt{2} \zeta\left[\left(\xi_{0}\right)^{2}-\vec{\xi} \cdot \vec{\xi}\right]
$$

For brevity we shall denote the element $\left(\zeta ; \xi_{0} \mathbf{1}+\vec{\xi} \cdot \vec{\sigma}\right)$ as

$$
\mathcal{X}=\left(\zeta ; \xi_{0}, \vec{\xi}\right)
$$

The three irreducible idempotents of $\mathbb{R} \oplus \boldsymbol{\Gamma}_{n+2}$ are

$$
\begin{aligned}
& E_{1} \equiv(1 ; 0, \overrightarrow{0}) \\
& E_{2} \equiv\left(0 ; \frac{1}{2}, \frac{1}{2}, 0, \ldots, 0\right) ; \\
& E_{3} \equiv\left(0 ; \frac{1}{2},-\frac{1}{2}, 0, . ., 0\right),
\end{aligned}
$$

with the identity element $\mathbf{\imath} \in \mathbb{R} \oplus \boldsymbol{\Gamma}_{n+2}$ given by

$$
\mathbf{\imath}=E_{1}+E_{2}+E_{3}=(1 ; 1, \overrightarrow{0}) .
$$

The automorphism group $A u t\left(\mathbb{R} \oplus \boldsymbol{\Gamma}_{n+2}\right)$ is $S O(n+1)$ and the reduced structure group $\operatorname{Str}_{0}\left(\mathbb{R} \oplus \boldsymbol{\Gamma}_{n+2}\right)$ is $S O(n+1,1) \otimes S O(1,1)$. The identity element $\mathbf{1}$ is manifestly invariant under $\operatorname{Aut}\left(\mathbb{R} \oplus \boldsymbol{\Gamma}_{n+2}\right)$, and the little group of the element $\mathfrak{b} \equiv-E_{1}-E_{2}+E_{3}=$ $(-1 ; 0,-1,0, \ldots, 0)$ is $S O(n, 1)$.

Let us now determine the explicit form of the generators $\mathcal{T}(A, B)$ (3.38) annihilating the corresponding reference vector in each class of charge orbits:

1. For

$$
(\alpha, \beta, x, y)=\kappa\left(1,0, E_{1}+E_{2}+E_{3}, 0\right)
$$

the annihilation condition

$$
\mathcal{T}(A, B) \kappa\left(1,0, E_{1}+E_{2}+E_{3}, 0\right)=0
$$

requires

$$
\left\{\begin{array}{l}
\alpha^{\prime}=0 \\
\beta=0 \Rightarrow \beta^{\prime}=0=\operatorname{Tr}(B) \\
x^{\prime}=0 \\
y=0 \Rightarrow y^{\prime}=0=-A+B
\end{array}\right.
$$


implying that

$$
A=B, \quad \operatorname{Tr}(A)=0 .
$$

Consequently, the generators $\mathcal{T}(A, A)$ with $\operatorname{Tr}(A)=0$ annihilate the reference vector (3.53). They correspond to $\operatorname{dim}(\mathcal{J})-1=n_{V}-1=\widehat{n}=\operatorname{dim}_{\mathbb{R}}\left(\frac{G_{5}}{H_{5}}\right)$ non-compact (Hermitian) generators outside $\mathfrak{S} \mathfrak{t r}(\mathcal{J})$.

The subgroup of $\operatorname{Str}_{0}(\mathcal{J})$ that leaves the reference vector (3.53) invariant is $\operatorname{Aut}(\mathcal{J})=$ $H_{5}$, which is compact. Hence, the stability group of the reference vector (3.53) is noncompact and isomorphic (not identical) to the reduced structure group $\operatorname{Str}_{0}(\mathcal{J})$; we shall denote it as $\operatorname{Str}_{0}^{*}(\mathcal{J})$.

Therefore, the corresponding non-degenerate charge orbits, listed in Table 4, are of the form

$$
\frac{\operatorname{Aut}(\mathcal{F}(\mathcal{J}))}{\operatorname{Str}_{0}^{*}(\mathcal{J})}=\frac{G_{4} \equiv G}{\widehat{H}}
$$

with m.c.s. $\left(\operatorname{Str}_{0}^{*}(\mathcal{J})\right)=\operatorname{Aut}(\mathcal{J})=\widehat{h}=H_{5}$.

Now, the quartic invariant of a vector of the form $\left(\alpha, 0,\left(\lambda_{1} E_{1}+\lambda_{2} E_{2}+\lambda_{3} E_{3}\right), 0\right) \in$ $\mathcal{F}(\mathcal{J})$ is simply

$$
I_{4}=-\alpha \lambda_{1} \lambda_{2} \lambda_{3} \equiv \lambda_{0} \lambda_{1} \lambda_{2} \lambda_{3}
$$

where we identify $-\alpha \equiv \lambda_{0}$. Furthermore, using the dilatation symmetry one can rescale $\alpha$ relative to the eigenvalues $\lambda_{i}$, and hence one can assume $\alpha=1$ without any loss of generality.

Thus, the orbits $\frac{\operatorname{Aut}(\mathcal{F}(\mathcal{J}))}{\operatorname{Str}(\mathcal{J})}$ have negative quartic invariant

$$
I_{4}=-\kappa^{4}=-\lambda_{1} \lambda_{2} \lambda_{3}<0,
$$

and a single negative "eigenvalue" $\lambda_{0}=-1$.

As it can be seen at a glance, such orbits can be interpreted as the non-BPS, $Z \neq 0$ ones (the right column of Table 4 coincides with the column of $\mathcal{O}_{n o n-B P S, Z \neq 0}$ orbits in Table 3).

2. For

$$
(\alpha, \beta, x, y)=\kappa\left(1,0,-E_{1}-E_{2}-E_{3}, 0\right)
$$

the annihilation condition

$$
\mathcal{T}(A, B) \kappa\left(1,0,-E_{1}-E_{2}-E_{3}, 0\right)=0
$$

requires

$$
\left\{\begin{array}{l}
\alpha^{\prime}=0 \\
\beta=0 \Rightarrow \beta^{\prime}=0=-\operatorname{Tr}(B) \\
x^{\prime}=0 \\
y=0 \Rightarrow y^{\prime}=0=A+B
\end{array}\right.
$$




\begin{tabular}{|c||c|c|}
\hline & $\mathcal{J}$ & $\frac{\operatorname{Aut}(\mathcal{F}(\mathcal{J}))}{S t r_{0}^{*}(\mathcal{J})}$ \\
\hline \hline$I I$ & $\mathbb{R} \oplus \boldsymbol{\Gamma}_{n+2}$ & $\frac{S U(1,1) \otimes S O(2,2+n)}{S O(1,1) \otimes S O(1,1+n)}$ \\
\hline$I I I$ & $J_{3}^{\mathbb{O}}$ & $\frac{E_{7(-25)}}{E_{6(-26)}}$ \\
\hline$I V$ & $J_{3}^{\mathbb{H}}$ & $\frac{S O^{*}(12)}{S U^{*}(6)}$ \\
\hline$V$ & $J_{3}^{\mathbb{C}}$ & $\frac{S U(3,3)}{S L(3, \mathbb{C})}$ \\
\hline$V I$ & $J_{3}^{\mathbb{R}}$ & $\frac{S p(6, \mathbb{R})}{S L(3, \mathbb{R})}$ \\
\hline
\end{tabular}

Table 4: Class $\frac{A u t(\mathcal{F}(\mathcal{J}))}{S t r_{0}^{*}(\mathcal{J})}$ of non-degenerate charge orbits with a negative quartic invariant and a single negative "eigenvalue" for all $N=2, d=4$ MESGTs defined by Euclidean Jordan algebras of degree three.

implying that

$$
A=-B, \quad \operatorname{Tr}(A)=0 .
$$

Consequently, the generators $\mathcal{T}(A,-A)$ with $\operatorname{Tr}(A)=0$ annihilate the reference vector (3.60). They correspond to $\operatorname{dim}(\mathcal{J})-1$ compact (anti-Hermitian) generators outside $\mathfrak{S t r}(\mathcal{J})$.

The subgroup of $\operatorname{Str}_{0}(\mathcal{J})$ that leaves the reference vector (3.60) invariant is, as in case 1 , the compact automorphism group $A u t(\mathcal{J})$. Thus, the stability group of the reference vector (3.60) is the compact form of $\operatorname{Str}_{0}(\mathcal{J})$, which we denote as $\operatorname{Ktr}_{0}(\mathcal{J})$. Therefore, the corresponding non-degenerate charge orbits are of the form

$$
\frac{\operatorname{Aut}(\mathcal{F}(\mathcal{J}))}{K \operatorname{tr}_{0}(\mathcal{J})}=\frac{G_{4} \equiv G}{H_{0}}
$$

with $K \operatorname{tr}_{0}(\mathcal{J})=\frac{\text { m.c.s. }(\operatorname{Aut}(\mathcal{F}(\mathcal{J})))}{U(1)}=H_{0}$. They have positive quartic invariant

$$
I_{4}=\kappa^{4}=\left|\lambda_{1} \lambda_{2} \lambda_{3}\right|>0
$$

and all four "eigenvalues" negative.

These orbits correspond to the $N=2, \frac{1}{2}$-BPS orbits, and they are listed in Table 5 (whose right column coincides with the column of $\mathcal{O}_{\frac{1}{2}-B P S}$ orbits in Table 3 ). 


\begin{tabular}{|c|c|c|}
\hline & $\mathcal{J}$ & $\frac{\operatorname{Aut}(\mathcal{F}(\mathcal{J}))}{K \operatorname{tr}_{0}(\mathcal{J})}$ \\
\hline$I I$ & $\mathbb{R} \oplus \boldsymbol{\Gamma}_{n+2}$ & $\frac{S U(1,1) \otimes S O(2,2+n)}{S O(2) \otimes S O(2+n)}$ \\
\hline$I I I$ & $J_{3}^{\mathbb{Q}}$ & $\frac{E_{7(-25)}}{E_{6}}$ \\
\hline$I V$ & $J_{3}^{\mathbb{H}}$ & $\frac{S O^{*}(12)}{S U(6)}$ \\
\hline$V$ & $J_{3}^{\mathbb{C}}$ & $\frac{S U(3,3)}{S U(3) \otimes S U(3)}$ \\
\hline$V I$ & $J_{3}^{\mathbb{R}}$ & $\frac{S p(6, \mathbb{R})}{S U(3)}$ \\
\hline
\end{tabular}

Table 5: Class $\frac{A u t(\mathcal{F}(\mathcal{J}))}{K t r_{0}(\mathcal{J})}$ of non-degenerate charge orbits with positive quartic invariant and all negative "eigenvalues" for all $N=2, d=4$ symmetric MESGTs defined by Euclidean Jordan algebras of degree three.

3. For

$$
(\alpha, \beta, x, y)=\kappa\left(1,0,-E_{1}-E_{2}+E_{3}, 0\right)
$$

the annihilation condition

$$
\mathcal{T}(A, B) \kappa\left(1,0,-E_{1}-E_{2}+E_{3}, 0\right)=0
$$

requires $\alpha^{\prime}=0, x^{\prime}=0$ and

$$
\left\{\begin{array}{l}
\beta=0 \Rightarrow \beta^{\prime}=0=T(B, x)=\operatorname{Tr}\left(B \circ\left(-E_{1}-E_{2}+E_{3}\right)\right) \\
y=0 \Rightarrow y^{\prime}=0=2 A \wedge x+B=2 A \wedge\left(-E_{1}-E_{2}+E_{3}\right)+B .
\end{array}\right.
$$

For generic elements $A, B$ belonging to the simple Euclidean Jordan algebra of degree three $J_{3}^{\mathbb{A}}$ (where $\mathbb{A}=\mathbb{O}, \mathbb{H}, \mathbb{C}, \mathbb{R}$ ), the general solution of Eqs. (3.68) yields noncompact (Hermitian) generators of the form $\mathcal{T}(A, A)$ with (the $3 \times 3$ Hermitian matrix representation of $A$ reading)

$$
A=\left(\begin{array}{ccc}
\alpha_{1} & a_{3} & 0 \\
a_{3}^{*} & \alpha_{2} & 0 \\
0 & 0 & \alpha_{1}+\alpha_{2}
\end{array}\right), \operatorname{dim}_{\mathbb{R}}(A)=2+\operatorname{dim}_{\mathbb{R}}(\mathbb{A})
$$


and compact (anti-Hermitian) generators of the form $\mathcal{T}(B,-B)$ with (the $3 \times 3$ Hermitian matrix representation of $B$ reading)

$$
B=\left(\begin{array}{ccc}
0 & 0 & a_{2}^{*} \\
0 & 0 & a_{1} \\
a_{2} & a_{1}^{*} & 0
\end{array}\right), \operatorname{dim}_{\mathbb{R}}(B)=2 \operatorname{dim}_{\mathbb{R}}(\mathbb{A}) .
$$

$a_{1}, a_{2}$ and $a_{3}$ are elements of the division algebra $\mathbb{A}$, whereas $\alpha_{1}$ and $\alpha_{2}$ are real numbers.

The subgroup of $\operatorname{Str}_{0}(\mathcal{J})$ that leaves the reference vector (3.66) invariant is a (noncompact) real form of $A u t(\mathcal{J})$, which we will denote as $\breve{H}(\mathcal{J})$.

The non-compact generators $\mathcal{T}(A, A)$ and the compact generators $\mathcal{T}(B,-B)$ (with $A$ and $B$ respectively given by Eqs. (3.69) and (3.70) ) form a representation of $\breve{H}(\mathcal{J}) \varsubsetneqq$ $\operatorname{Str}_{0}(\mathcal{J})$ of dimension

$$
\operatorname{dim}_{\mathbb{R}}(A)+\operatorname{dim}_{\mathbb{R}}(B)=2+3 \operatorname{dim}_{\mathbb{R}}(\mathbb{A})=\operatorname{dim}(\mathcal{J})-1
$$

Furthermore, the non-compact generators $\mathcal{T}(A, A)$, the compact generators $\mathcal{T}(B,-B)$ (with $A$ and $B$ respectively given by Eqs. (3.69) and (3.70) ) and the generators of $\breve{H}(\mathcal{J})$ all together generate the non-compact stability group of the reference vector (3.66), which we will denote as $\Sigma_{0}(\mathcal{J})$.

A similar treatment can be given also for the reducible case $\mathcal{J}=\mathbb{R} \oplus \boldsymbol{\Gamma}_{n+2}$. By also using the results of [1, 27], in Table 6 we list the groups $\breve{H}(\mathcal{J})$ and $\Sigma_{0}(\mathcal{J})$ for all the symmetric $N=2, d=4$ MESGTs related to an Euclidean Jordan algebra of degree three $\mathcal{J}$.

Since $\Sigma_{0}(\mathcal{J}) \sim \operatorname{Str}_{0}^{*}(\mathcal{J})$, the charge orbits of case 1 and the charge orbits $\frac{\operatorname{Aut}(\mathcal{F}(\mathcal{J}))}{\Sigma_{0}(\mathcal{J})}$ of the present case overlap:

$$
\frac{G_{4} \equiv G}{\widehat{H}}=\frac{\operatorname{Aut}(\mathcal{F}(\mathcal{J}))}{\operatorname{Str}_{0}^{*}(\mathcal{J})} \sim \frac{\operatorname{Aut}(\mathcal{F}(\mathcal{J}))}{\Sigma_{0}(\mathcal{J})} .
$$

This is consistent with the result that the corresponding reference vectors (3.53) and (3.66) can be mapped into each other by the action of $A u t(\mathcal{F}(\mathcal{J}))$, as discussed above $\left(\kappa_{1}, \kappa_{3} \in \mathbb{R}_{0}^{+}\right)$:

$$
\kappa_{1}\left(1,0, E_{1}+E_{2}+E_{3}, 0\right) \stackrel{\operatorname{Aut}(\mathcal{F}(\mathcal{J}))}{\longleftrightarrow} \kappa_{3}\left(1,0,-E_{1}-E_{2}+E_{3}, 0\right) .
$$

The orbits $\frac{\operatorname{Aut}(\mathcal{F}(\mathcal{J}))}{\Sigma_{0}(\mathcal{J})}$ can be interpreted as the non-BPS, $Z \neq 0$ ones with a negative quartic invariant

$$
I_{4}=-4 \kappa^{4}=-4 \lambda_{1} \lambda_{2} \lambda_{3}<0,
$$

and with three negative "eigenvalues".

4. For

$$
(\alpha, \beta, x, y)=\kappa\left(1,0, E_{1}+E_{2}-E_{3}, 0\right)
$$




\begin{tabular}{|c||c|c|c|}
\hline & $\mathcal{J}$ & $\breve{H}(\mathcal{J})$ & $\Sigma_{0}(\mathcal{J})$ \\
\hline \hline$I I$ & $\mathbb{R} \oplus \boldsymbol{\Gamma}_{n+2}$ & $S O(n, 1)$ & $S O(n+1,1) \times S O(1,1)$ \\
\hline$I I I$ & $J_{3}^{\mathbb{Q}}$ & $F_{4(-20)}$ & $E_{6(-26)}$ \\
\hline$I V$ & $J_{3}^{\mathbb{H}}$ & $U S p(4,2)$ & $S U^{*}(6)$ \\
\hline$V$ & $J_{3}^{\mathbb{C}}$ & $S U(2,1)$ & $S L(3, \mathbb{C})$ \\
\hline$V I$ & $J_{3}^{\mathbb{R}}$ & $S L(2, \mathbb{R})$ & $S L(3, \mathbb{R})$ \\
\hline
\end{tabular}

Table 6: Non-compact group $\breve{H}(\mathcal{J}) \varsubsetneqq \operatorname{Str}_{0}(\mathcal{J})$ and non-compact stability group $\Sigma_{0}(\mathcal{J}) \sim \operatorname{Str}_{0}^{*}(\mathcal{J})$ of the reference vector $\kappa\left(1,0,-E_{1}-E_{2}+E_{3}, 0\right)$ of the FTSs corresponding to all $N=2, d=4$ MESGTs defined by Euclidean Jordan algebras of degree three.

the annihilation condition

$$
\mathcal{T}(A, B) \kappa\left(1,0, E_{1}+E_{2}-E_{3}, 0\right)=0
$$

requires $\alpha^{\prime}=0, x^{\prime}=0$ and

$$
\left\{\begin{array}{l}
\beta=0 \Rightarrow \beta^{\prime}=0=T(B, x)=-\operatorname{Tr}\left(B \circ\left(-E_{1}-E_{2}+E_{3}\right)\right) ; \\
y=0 \Rightarrow y^{\prime}=0=2 A \wedge x+B=-2 A \wedge\left(-E_{1}-E_{2}+E_{3}\right)+B .
\end{array}\right.
$$

For generic elements $A, B \in J_{3}^{\mathbb{A}}(\mathbb{A}=\mathbb{O}, \mathbb{H}, \mathbb{C}, \mathbb{R})$, the general solution of Eqs. (3.77) yields compact (anti-Hermitian) generators of the form $\mathcal{T}(A,-A)$ and non-compact (Hermitian) generators of the form $\mathcal{T}(B, B)$, with (the $3 \times 3$ Hermitian matrix representations of) $A$ and $B$ respectively given by Eqs. (3.69) and (3.70).

The subgroup of $\operatorname{Str}_{0}(\mathcal{J})$ that leaves the reference vector (3.75) invariant is $\breve{H}(\mathcal{J})$ as in case 3. However, in the present case the number of compact and non-compact generators outside $\mathfrak{S t r}(\mathcal{J})$ is respectively equal to $2+\operatorname{dim}_{\mathbb{R}}(\mathbb{A})$ and $2 \operatorname{dim}_{\mathbb{R}}(\mathbb{A})$, i.e. the opposite of what happens in case 3 .

Furthermore, the compact generators $\mathcal{T}(A,-A)$, the non-compact generators $\mathcal{T}(B, B)$ (with $A$ and $B$ respectively given by Eqs. (3.69) and (3.70) $)$ and the generators of $\breve{H}(\mathcal{J})$ 


\begin{tabular}{|c||c|c|c|}
\hline & $\mathcal{J}$ & $\breve{\mathcal{H}}(\mathcal{J})$ & $\frac{\operatorname{Aut}(\mathcal{F}(\mathcal{J}))}{\Delta_{0}(\mathcal{J})}$ \\
\hline \hline$I I$ & $\mathbb{R} \oplus \boldsymbol{\Gamma}_{n+2}$ & $S O(n, 1)$ & $\frac{S U(1,1) \otimes S O(2,2+n)}{S O(2) \otimes S O(2, n)}$ \\
\hline$I I I$ & $J_{3}^{\mathbb{O}}$ & $F_{4(-20)}$ & $\frac{E_{7(-25)}}{E_{6(-14)}}$ \\
\hline$I V$ & $J_{3}^{\mathbb{H}}$ & $U S p(4,2)$ & $\frac{S O^{*}(12)}{S U(4,2)}$ \\
\hline$V$ & $J_{3}^{\mathbb{C}}$ & $S U(2,1)$ & $\frac{S U(3,3)}{S U(2,1) \otimes S U(1,2)}$ \\
\hline$V I$ & $J_{3}^{\mathbb{R}}$ & $S L(2, \mathbb{R})$ & $\frac{S p(6, \mathbb{R})}{S U(2,1)}$ \\
\hline
\end{tabular}

Table 7: Non-compact group $\breve{H}(\mathcal{J})$ and class $\frac{A u t(\mathcal{F}(\mathcal{J}))}{\Delta_{0}(\mathcal{J})}$ of non-degenerate charge orbits with a positive quartic norm and two negative "eigenvalues" for all $N=2, d=4$ symmetric MESGTs defined by Euclidean Jordan algebras of degree three.

all together generate the non-compact stability group of the reference vector (3.75), which we will denote as $\Delta_{0}(\mathcal{J})$.

A similar treatment can be given also for the reducible case $\mathcal{J}=\mathbb{R} \oplus \boldsymbol{\Gamma}_{n+2}$ using results of [1, 27].

Therefore, the corresponding non-degenerate charge orbits are of the form

$$
\frac{\operatorname{Aut}(\mathcal{F}(\mathcal{J}))}{\Delta_{0}(\mathcal{J})}=\frac{G_{4} \equiv G}{\widetilde{H}}
$$

They have positive quartic invariant

$$
I_{4}=4 \kappa^{4}=4\left|\lambda_{1} \lambda_{2} \lambda_{3}\right|>0
$$

and two negative "eigenvalues".

By also using the results of [1, 27], in Table 7 we list the groups $\breve{\mathcal{H}}(\mathcal{J})$ and the orbits $\frac{\operatorname{Aut}(\mathcal{F}(\mathcal{J}))}{\Delta_{0}(\mathcal{J})}$ for all the symmetric $N=2, d=4$ MESGTs defined by Euclidean Jordan algebras of degree three $\mathcal{J}$. The orbits $\frac{A u t(\mathcal{F}(\mathcal{J}))}{\Delta_{0}(\mathcal{J})}$ correspond to the non-BPS, $Z=0$ ones (the right column of Table 7 coincides with the column of $\mathcal{O}_{n o n-B P S, Z=0}$ orbits in Table $3)$. 
Notice that, consistently with the previous treatment, the reference vectors (3.60) and (3.75) (both having $\lambda_{1} \lambda_{2} \lambda_{3}<0$ ) cannot be mapped into each other by $\operatorname{Aut}(\mathcal{F}(\mathcal{J})$ ), and therefore the corresponding non-degenerate charge orbits do not overlap $\left(\kappa_{2}, \kappa_{4} \in \mathbb{R}_{0}^{+}\right)$:

$$
\begin{gathered}
\kappa_{2}\left(1,0,-E_{1}-E_{2}-E_{3}, 0\right) \stackrel{\operatorname{Aut}(\mathcal{F}(\mathcal{J}))}{\leftrightarrow} \kappa_{4}\left(1,0, E_{1}+E_{2}-E_{3}, 0\right) \\
\Downarrow \\
\mathcal{O}_{\frac{1}{2}-B P S}=\frac{G_{4} \equiv G}{H_{0}}=\frac{A u t(\mathcal{F}(\mathcal{J}))}{K \operatorname{tr}_{0}(\mathcal{J})} \neq \frac{\operatorname{Aut}(\mathcal{F}(\mathcal{J}))}{\Delta_{0}(\mathcal{J})}=\frac{G_{4} \equiv G}{\widetilde{H}}=\mathcal{O}_{\text {non }-B P S, Z=0} .
\end{gathered}
$$

\section{Classification of Attractors}

The three classes of orbits in Table 3 correspond to the three distinct classes of solutions of the $N=2, d=4$ extremal black hole attractor equations (2.12) and (2.17).

\section{1 $\frac{1}{2}$-BPS solutions}

As already mentioned, the class of $\frac{1}{2}$-BPS orbits corresponds to the solution (2.18) determining $N=2, \frac{1}{2}$-BPS critical points of $V_{B H}$. Such a solution yields the following value of the black hole scalar potential at the considered attractor point(s) [14]:

$$
V_{B H, \frac{1}{2}-B P S}=|Z|_{\frac{1}{2}-B P S}^{2}+\left[G^{i \bar{i}} D_{i} Z \overline{D_{\bar{i}}} \bar{Z}\right]_{\frac{1}{2}-B P S}=|Z|_{\frac{1}{2}-B P S}^{2} \cdot
$$

The overall symmetry group at $N=2 \frac{1}{2}$-BPS critical point(s) is $H_{0}$, stabilizer of $\mathcal{O}_{\frac{1}{2}-B P S}=$ $\frac{G}{H_{0}}$. The symmetry enhancement is given by Eq. (2.19). For such a class of orbits

$$
I_{4, \frac{1}{2}-B P S}=|Z|_{\frac{1}{2}-B P S}^{4}>0 .
$$

\subsection{Non-BPS solutions}

The two classes of $N=2$ non-BPS non-degenerate charge orbits respectively correspond to the following solutions of $N=2$ attractor eqs. (2.12):

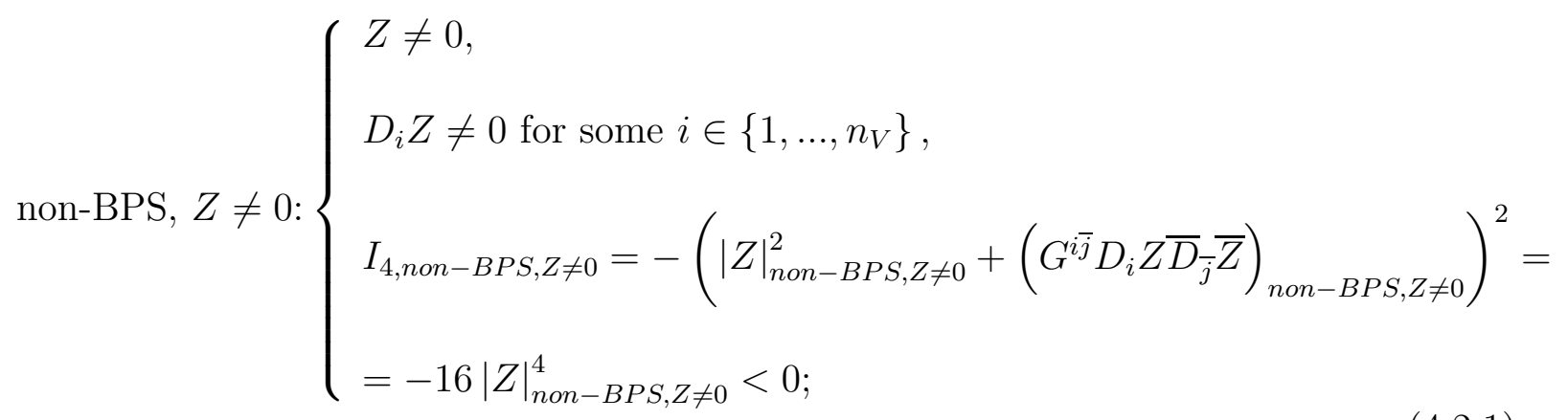




$$
\text { non-BPS, } Z=0:\left\{\begin{array}{l}
Z=0, \\
D_{i} Z \neq 0 \text { for some } i \in\left\{1, \ldots, n_{V}\right\}, \\
I_{4, n o n-B P S, Z=0}=\left(G^{i \bar{j}} D_{i} Z \bar{D} \bar{j} \bar{Z}\right)_{n o n-B P S, Z=0}^{2}>0 .
\end{array}\right.
$$

In Subsubsects. 4.2.1 and 4.2.2 we will show how the general solutions of Eqs. (2.12), respectively determining the two aforementioned classes of $N=2$ non-BPS extremal black hole attractors, can be easily given by using "flat" local $I$-coordinates in the scalar manifold.

Otherwise speaking, we will consider the "flatted" attractor eqs. (2.17), which can be specialized in the regular non-BPS cases as follows:

$$
\begin{aligned}
& \text { non-BPS, } Z \neq 0: \quad 2 \bar{Z} D_{I} Z=-i C_{I J K} \delta^{J \bar{J}} \delta^{K \bar{K}} \bar{D}_{\bar{J}} \overline{Z D}_{\bar{K}} \bar{Z} ; \\
& \text { non-BPS, } Z=0: \quad C_{I J K} \delta^{J \bar{J}} \delta^{K \bar{K}} \bar{D}_{\bar{J}} \overline{Z D}_{\bar{K}} \bar{Z}=0 .
\end{aligned}
$$

Thus, by respectively denoting with $\widehat{H}(\widetilde{H})$ the stabilizer of the $N=2$, non-BPS, $Z \neq 0$ $(Z=0)$ classes of orbits listed in Table 3, our claim is the following: the general solution of Eqs. (4.2.3) (4.2.4) ) is obtained by retaining a complex charge vector $\left(Z, D_{I} Z\right)$ which is invariant under $\widehat{h}\left(\frac{\widetilde{h}}{U(1)}\right)$, where $\widehat{h}(\widetilde{h})$ is the m.c.s. ${ }^{12}$ of $\widehat{H}(\widetilde{H})$.

As a consequence, the overall symmetry group of the $N=2$, non-BPS, $Z \neq 0(Z=0)$ critical point(s) is $\widehat{h}\left(\frac{\widetilde{h}}{U(1)}\right)$. Thus, at $N=2$, non-BPS, $Z \neq 0(Z=0)$ critical point(s) the following enhancement of symmetry holds:

$$
\begin{aligned}
& N=2, \text { non-BPS, } Z \neq 0: \mathcal{S} \longrightarrow \widehat{h}=m . c . s .(\widehat{H}) \\
& N=2, \text { non-BPS, } Z=0: \mathcal{S} \longrightarrow \frac{\widetilde{h}}{U(1)}=\frac{m . c . s .(\widetilde{H})}{U(1)} .
\end{aligned}
$$

It is worth remarking that the non-compact group $\widehat{H}$ stabilizing the non-BPS, $Z \neq 0$ class of orbits of $N=2, d=4$ symmetric MESGTs, beside being a real (non-compact) form of $H_{0}$, is isomorphic to the duality group $G_{5}$ of $N=2, d=5$ symmetric MESGTs

\footnotetext{
${ }^{12}$ Indeed, while $H_{0}$ is a proper compact subgroup of $G$, the groups $\widehat{H}, \widetilde{H}$ are real (non-compact) forms of $H_{0}$, as it can be seen from Table 3 (see also [67, 68]). Therefore in general they admit a m.c.s. $\widehat{h}, \widetilde{h}$, which in turn is a (non-maximal) compact subgroup of $G$ and a proper compact subgroup of $H_{0}$.

It is interesting to notice that in all cases (listed in Table 3$) G$ always admits only 2 real (non-compact) forms $\widehat{H}, \widetilde{H}$ of $H_{0}$ as proper subgroups (consistent with the required dimension of orbits). The inclusion of $\widehat{H}, \widetilde{H}$ in $G$ is such that in all cases $\widehat{H} \otimes S O(1,1)$ and $\widetilde{H} \otimes U(1)$ are different maximal non-compact subgroups of $G$.
} 


\begin{tabular}{|c|c|c|c|c|c|}
\hline & $H_{0}$ & $\widehat{H}$ & $\widetilde{H}$ & $\widehat{h} \equiv$ m.c.s. $(\widehat{H})$ & $\widetilde{h}^{\prime} \equiv \frac{m . c . s .(\widetilde{H})}{U(1)}$ \\
\hline I & $S U(n+1)$ & - & $S U(1, n)$ & - & $S U(n)$ \\
\hline II & $\begin{array}{c}S O(2) \\
\otimes \\
S O(2+n)\end{array}$ & $\begin{array}{c}S O(1,1) \\
\otimes \\
S O(1,1+n)\end{array}$ & $\begin{array}{c}S O(2) \\
\otimes \\
S O(2, n)\end{array}$ & $S O(1+n)$ & $\begin{array}{c}S O(2) \\
\otimes \\
S O(n)\end{array}$ \\
\hline III & $E_{6} \equiv E_{6(-78)}$ & $E_{6(-26)}$ & $E_{6(-14)}$ & $F_{4} \equiv F_{4(-52)}$ & $S O(10)$ \\
\hline$I V$ & $S U(6)$ & $S U^{*}(6)$ & $S U(4,2)$ & $U S p(6)$ & $\begin{array}{c}S U(4) \\
\otimes \\
S U(2)\end{array}$ \\
\hline$V$ & $S U(3) \otimes S U(3)$ & $S L(3, \mathbb{C})$ & $\begin{array}{c}S U(2,1) \\
\otimes \\
S U(1,2)\end{array}$ & $S U(3)$ & $\begin{array}{c}S U(2) \\
\otimes \\
S U(2) \otimes U(1)\end{array}$ \\
\hline$V I$ & $S U(3)$ & $S L(3, \mathbb{R})$ & $S U(2,1)$ & $S O(3)$ & $S U(2)$ \\
\hline
\end{tabular}

Table 8: Stabilizers and corresponding m.c.s.s of the non-degenerate classes of orbits of $N=2, d=4$ symmetric MESGTs. $\widehat{H}$ and $\widetilde{H}$ are real (non-compact) forms of $H_{0}$, the stabilizer of $\frac{1}{2}$-BPS orbits.

and was denoted as $\operatorname{Str}_{0}^{*}(\mathcal{J})$ in Sect. 3 (see Table 4) ${ }^{13}$. Consequently, in the cases $I I-V I$ of Table 3, Eq. (3.15) can be completed as follows:

$$
H_{5}=\operatorname{Aut}(\mathcal{J})=\text { m.c.s. }\left(G_{5}\right) \varsubsetneqq \widehat{H}=\operatorname{Str}_{0}^{*}(\mathcal{J}) \varsubsetneqq \operatorname{Aut}(\mathcal{F}(\mathcal{J}))=G \equiv G_{4} .
$$

Since the scalar manifolds of $N=2, d=5$ symmetric MESGTs are endowed with a real special geometry (see Footnote 10) [37, 38, 39], the complex representation $R_{H_{0}}$ of $H_{0}$ decomposes in a pair of irreducible real representations $\left(R_{\widehat{h}}+1\right)_{\mathbb{R}}$ 's of $\widehat{h}=$ m.c.s. $(\widehat{H}) \varsubsetneqq$ $H_{0}$ (see Subsubsect. 4.2.1] and in particular Eq. (4.2.1.1)). As we will see in Subsubsects. 4.2 .1 and 4.2.2 such a fact crucially distinguishes the non-BPS, $Z \neq 0$ and $Z=0$ cases.

The stabilizers (and the corresponding m.c.s.s) of the non-BPS, $Z \neq 0$ and $Z=0$ classes of orbits of $N=2, d=4$ symmetric MESGTs are given in Table 8.

\footnotetext{
${ }^{13}$ Such a feature is missing in the $N=2, d=4$ symmetric MESGTs whose scalar manifolds belong to the sequence $I$, simply because such theories do not have a class of non-BPS, $Z \neq 0$ orbits.
} 


\subsubsection{Non-BPS, $Z \neq 0$ solutions}

Let us start by considering the class of non-BPS, $Z \neq 0$ orbits of $N=2, d=4$ symmetric MESGTs.

As mentioned, the "flatted matter charges" $D_{I} Z$ are a vector of $R_{H_{0}}$. In general, $R_{H_{0}}$ decomposes under the m.c.s. $\widehat{h} \subset \widehat{H}$ as follows:

$$
R_{H_{0}} \longrightarrow\left(R_{\widehat{h}}+\mathbf{1}\right)_{\mathbb{C}}
$$

where the r.h.s. is made of the complex singlet representation of $\widehat{h}$ and by another nonsinglet real representation of $\widehat{h}$, denoted above with $R_{\widehat{h}}$. As previously mentioned, despite being complex, $\left(R_{\widehat{h}}+\mathbf{1}\right)_{\mathbb{C}}$ is not charged with respect to $U(1)$ symmetry because, due to the 5 -dimensional origin of the non-compact stabilizer $\widehat{H}$ whose m.c.s. is $\widehat{h}$, actually $\left(R_{\widehat{h}}+\mathbf{1}\right)_{\mathbb{C}}$ is nothing but the complexification of its real counterpart $\left(R_{\widehat{h}}+\mathbf{1}\right)_{\mathbb{R}}$. The decomposition (4.2.1.1)yields the following splitting of "flatted matter charges":

$$
D_{I} Z \longrightarrow\left(D_{\widehat{I}} Z, D_{\widehat{I}_{0}} Z\right)
$$

where $\widehat{I}$ are the indices along the representation $R_{\widehat{h}}$, and $\widehat{I}_{0}$ is the $\widehat{h}$-singlet index.

By considering the related attractor eqs., it should be noticed that the rank-3 symmetric tensor $C_{I J K}$ in Eqs. (4.2.3) corresponds to a cubic $H_{0}$-invariant coupling $\left(R_{H_{0}}\right)^{3}$. By decomposing $\left(R_{H_{0}}\right)^{3}$ in terms of representations of $\widehat{h}$, one finds

$$
\left(R_{H_{0}}\right)^{3} \longrightarrow\left(R_{\widehat{h}}\right)^{3}+\left(R_{\widehat{h}}\right)^{2} \mathbf{1}_{\mathbb{C}}+\left(\mathbf{1}_{\mathbb{C}}\right)^{3} .
$$

Notice that a term $R_{\widehat{h}}\left(\mathbf{1}_{\mathbb{C}}\right)^{2}$ cannot be in such a representation decomposition, since it is not $\widehat{h}$-invariant, and thus not $H_{0}$-invariant. This implies that components of the form $C_{\widehat{I} \widehat{I}_{0} \widehat{I}_{0}}$ cannot exist. Also, a term like $\left(\mathbf{1}_{\mathbb{C}}\right)^{3}$ can appear in the r.h.s. of the decomposition (4.2.3) since as we said the $\widehat{h}$-singlet $\mathbf{1}_{\mathbb{C}}$, despite being complex, is not $U(1)$-charged.

It is then immediate to conclude that the solution of $N=2, d=4$ non-BPS, $Z \neq 0$ extremal black hole attractor eqs. in "flat" indices (4.2.3) corresponds to keep the "flatted matter charges" $D_{I} Z \widehat{h}$-invariant. By virtue of decomposition (4.2.1.3), this is obtained by putting

$$
D_{\widehat{I}} Z=0, D_{\widehat{I}_{0}} Z \neq 0,
$$

i.e. by putting all "flatted matter charges" to zero, except the one along the $\widehat{h}$-singlet (and thus $\widehat{h}$-invariant) direction in scalar manifold. By substituting the solution (4.2.1.4) 
in Eqs. (4.2.3), one obtains

$$
\begin{aligned}
& 2 \bar{Z} D_{\widehat{I}_{0}} Z=-i C_{\widehat{I}_{0} \widehat{I}_{0} \widehat{I}_{0}}\left(\bar{D} \overline{\widehat{I}}_{0} \bar{Z}\right)^{2} \stackrel{Z \neq 0}{\Leftrightarrow} D_{\widehat{I}_{0}} Z=-\frac{i}{2} \frac{C_{\widehat{I}_{0} \widehat{I}_{0} \widehat{I}_{0}}}{\bar{Z}}\left(\bar{D}{\overline{\widehat{I}_{0}}}_{\bar{Z}}\right)^{2} \\
& \Downarrow \\
& \left|D_{\widehat{I}_{0}} Z\right|^{2}\left(1-\frac{1}{4} \frac{\left|C_{\widehat{I}_{0} \widehat{I}_{0} \widehat{I}_{0}}\right|^{2}}{|Z|^{2}}\left|D_{\widehat{I}_{0}} Z\right|^{2}\right)=0 \\
& \Uparrow \\
& \left|D_{\widehat{I}_{0}} Z\right|^{2}=4 \frac{|Z|^{2}}{\left|C_{\widehat{I}_{0} \widehat{I}_{0} \widehat{I}_{0}}\right|^{2}} ;
\end{aligned}
$$

this is nothing but the general criticality condition of $V_{B H}$ for the 1-modulus case in the locally "flat" coordinate $\widehat{I}_{0}$, which in this case corresponds to the $\widehat{h}$-singlet direction in the scalar manifold. Such a case has been thoroughly studied in non-flat $i$-coordinates in [54.

All $N=2, d=4$ symmetric MESGTs (disregarding the sequence $I$ having $C_{i j k}=0$ ) have a cubic prepotential ( $F=\frac{1}{3 !} d_{i j k} t^{i} t^{j} t^{k}$ in special coordinates), and thus in special coordinates it holds that $C_{i j k}=e^{K} d_{i j k}$, with $K$ and $d_{i j k}$ respectively denoting the Kähler potential and the completely symmetric rank-3 constant tensor that is determined by the norm form of the underlying Jordan algebra of degree three [38. In the cubic $n_{V}=1$ modulus case, by using Eq. (2.15) it follows that

$$
\left(G^{1_{s} 1_{s}}\right)^{3}\left|C_{1_{s} 1_{s} 1_{s}}\right|^{2}=\left|C_{1_{f} 1_{f} 1_{f}}\right|^{2}=\frac{4}{3},
$$

where the subscripts "s" and "f" respectively stand for "special" and "flat", denoting the kind of coordinate system being considered. By substituting Eq. (4.2.1.7) in Eq. (4.2.1.6) one obtains the result

$$
\left|D_{\widehat{I}_{0}} Z\right|^{2}=3|Z|^{2}
$$

Another way of proving Eq. (4.2.1.8) is by computing the quartic invariant along the $\widehat{h}$-singlet direction, then yielding

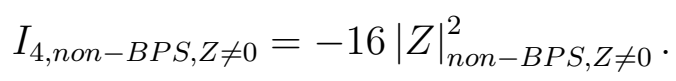

The considered solution (4.2.1.4)-(4.2.1.6), (4.2.1.8) is the $N=2$ analogue of the $N=8, d=4$ non-BPS regular solution discussed in [26], and it yields the following value of the black hole scalar potential at the considered attractor point(s) [54, 26]:

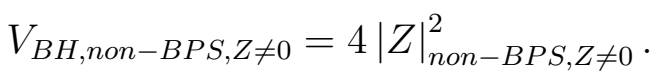

Once again, as for the non-BPS $N=8$ regular solutions (see Eq. (1.9)), we find the extra factor 4 .

From the above considerations, the overall symmetry group at $N=2$ non-BPS, $Z \neq 0$

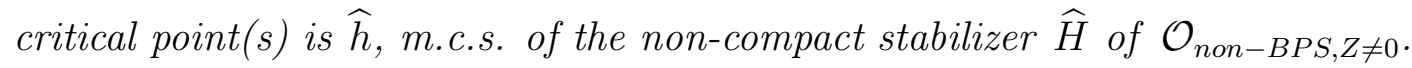




\subsubsection{Non-BPS, $Z=0$ solutions}

Let us now move to consider the other class of non-BPS orbits of $N=2, d=4$ symmetric MESGTs.

It has $Z=0$ and it was not considered in 1] (see also Footnote 7). We will show that the solution of the $N=2, d=4$, non-BPS, $Z=0$ extremal black hole attractor eqs. (4.2.4) are the "flatted matter charges" $D_{I} Z$ which are invariant under $\frac{\widetilde{h}}{U(1)}$, where $\widetilde{h}$ is the m.c.s. of $\widetilde{H}$, the stabilizer of the class $\mathcal{O}_{\text {non-BPS,Z=0 }}=\frac{G}{\widetilde{H}}$.

Differently from the non-BPS, $Z \neq 0$ case, in the considered non-BPS, $Z=0$ case there is always a $U(1)$ symmetry acting, since the scalar manifolds of $N=2, d=4$ symmetric MESGTs all have the group $\widetilde{h}$ of the form

$$
\widetilde{h}=\widetilde{h}^{\prime} \otimes U(1), \quad \widetilde{h}^{\prime} \equiv \frac{\widetilde{h}}{U(1)} .
$$

The compact subgroups $\widetilde{h}^{\prime}$ for all $N=2, d=4$ symmetric MESGTs are listed in Table 8. In the case at hand, we thence have to consider the decomposition of the previously introduced complex representation $R_{H_{0}}$ under the compact subgroup $\widetilde{h}^{\prime} \varsubsetneqq H_{0}$. In general, $R_{H_{0}}$ decomposes under $\widetilde{h}^{\prime} \varsubsetneqq \widetilde{H}$ as follows:

$$
R_{H_{0}} \longrightarrow\left(\mathcal{W}_{\widetilde{h}^{\prime}}+\mathcal{Y}_{\widetilde{h}^{\prime}}+1\right)_{\mathbb{C}}
$$

where in the r.h.s. the complex singlet representation of $\widetilde{h}^{\prime}$ and two complex non-singlet representations $\mathcal{W}_{\widetilde{h}^{\prime}}$ and $\mathcal{Y}_{\widetilde{h}^{\prime}}$ of $\widetilde{h}^{\prime}$ appear. In general, $\mathcal{W}_{\widetilde{h}^{\prime}}, \mathcal{Y}_{\widetilde{h}^{\prime}}$ and $\mathbf{1}_{\mathbb{C}}$ are charged (and thus not invariant) with respect to the $U(1)$ explicit factor appearing in (4.2.2.1). The decomposition (4.2.2.2) yields the following splitting of "flatted matter charges":

$$
D_{I} Z \longrightarrow\left(D_{\widetilde{I}_{\mathcal{W}}^{\prime}} Z, D_{\widetilde{I}_{\mathcal{Y}}^{\prime}} Z, D_{\widetilde{I}_{0}^{\prime}} Z\right)
$$

where $\widetilde{I}_{\mathcal{W}}^{\prime}$ and $\widetilde{I}_{\mathcal{Y}}^{\prime}$ respectively denote the indices along the complex representations $\mathcal{W}_{\widetilde{h}^{\prime}}$ and $\mathcal{Y}_{\widetilde{h}^{\prime}}$, and $\widetilde{I}_{0}^{\prime}$ is the $\widetilde{h}^{\prime}$-singlet index.

Once again, the related $N=2, d=4$ non-BPS, $Z=0$ extremal black hole attractor eqs. (4.2.4) contain the rank-3 symmetric tensor $C_{I J K}$, corresponding to a cubic $H_{0^{-}}$ invariant coupling $\left(R_{H_{0}}\right)^{3}$. The decomposition of $\left(R_{H_{0}}\right)^{3}$ in terms of representations of $\widetilde{h}^{\prime}$ yields

$$
\left(R_{H_{0}}\right)^{3} \longrightarrow\left(\mathcal{W}_{\widetilde{h}^{\prime}}\right)^{2} \mathcal{Y}_{\widetilde{h}^{\prime}}+\left(\mathcal{Y}_{\widetilde{h}^{\prime}}\right)^{2} \mathbf{1}_{\mathbb{C}}
$$

When decomposed under $\widetilde{h}^{\prime},\left(R_{H_{0}}\right)^{3}$ must be nevertheless $\widetilde{h}$-invariant, and therefore, beside the $\widetilde{h}^{\prime}$-invariance, one has to consider the invariance under the $U(1)$ factor, too. Thus, terms of the form $\left(\mathcal{W}_{\widetilde{h}^{\prime}}\right)^{3},\left(\mathcal{Y}_{\widetilde{h}^{\prime}}\right)^{3}, \mathcal{W}_{\widetilde{h}^{\prime}}\left(\mathbf{1}_{\mathbb{C}}\right)^{2}, \mathcal{Y}_{\widetilde{h}^{\prime}}\left(\mathbf{1}_{\mathbb{C}}\right)^{2}$ and $\left(\mathbf{1}_{\mathbb{C}}\right)^{3}$ cannot exist in the $\widetilde{h}$-invariant r.h.s. of decomposition (4.2.2.4).

Notice also that the structure of the decomposition (4.2.2.4) implies that components of the cubic coupling of the form $C_{\widetilde{I}_{\mathcal{W}}^{\prime} \widetilde{I}_{0}^{\prime} \widetilde{I}_{0}^{\prime}}, C_{\widetilde{I}_{\mathcal{Y}}^{\prime} \widetilde{I}_{0}^{\prime} \widetilde{I}_{0}^{\prime}}$ and $C_{\widetilde{I}_{0}^{\prime} \widetilde{I}_{0}^{\prime} \widetilde{I}_{0}^{\prime}}$ cannot exist. For such a 
reason, it is immediate to conclude that the solution of $N=2, d=4$ non-BPS, $Z=0$ extremal black hole attractor eqs. in "flat" indices (4.2.4) corresponds to keep the "flatted matter charges" $D_{I} Z \widetilde{h}^{\prime}$-invariant. By virtue of decomposition (4.2.2.4), this is obtained by putting

$$
D_{\widetilde{I}_{\mathcal{W}}^{\prime}} Z=0=D_{\widetilde{I}_{\mathcal{Y}}^{\prime}} Z, \quad D_{\widetilde{I}_{0}^{\prime}} Z \neq 0
$$

i.e. by putting all "flatted matter charges" to zero, except the one along the $\widetilde{h}^{\prime}$-singlet (and thus $\widetilde{h}^{\prime}$-invariant, but not $U(1)$-invariant and therefore not $\widetilde{h}$-invariant) direction in the scalar manifold.

The considered solution (4.2.2.5) does not have any analogue in $N=8, d=4$ SUGRA, and it yields the following value of the black hole scalar potential at the considered attractor point(s):

$$
\begin{aligned}
V_{B H, n o n-B P S, Z=0} & =|Z|_{n o n-B P S, Z=0}^{2}+\left[G^{i \bar{i}} D_{i} Z \bar{D} \bar{i} \bar{Z}\right]_{n o n-B P S, Z=0}= \\
& =\left|D_{\widetilde{I}_{0}^{\prime}} Z\right|_{n o n-B P S, Z=0}^{2} \cdot
\end{aligned}
$$

It is here worth remarking that in the stu model it can be explicitly computed that 69.

$$
V_{B H, n o n-B P S, Z=0}=\left|D_{\widetilde{I}_{0}^{\prime}} Z\right|_{n o n-B P S, Z=0}^{2}=|Z|_{\frac{1}{2}-B P S}^{2}=V_{B H, \frac{1}{2}-B P S}
$$

From above considerations, the overall symmetry group at $N=2$ non-BPS, $Z=0$

critical point $(s)$ is $\widetilde{h}^{\prime}=\frac{\widetilde{h}}{U(1)}, \widetilde{h}$ being the m.c.s. of the non-compact stabilizer $\widetilde{H}$ of $\mathcal{O}_{\text {non }-B P S, Z=0}$.

The general analysis carried out above holds for all $N=2, d=4$ symmetric "magical" MESGTs, namely for the irreducible cases $I I I-V I$ listed in Tables 2 and 3. The cases of irreducible sequence $I$ and of generic Jordan family $I I$ deserve suitable, slightly different treatments, respectively given in Appendices I and II.

\subsection{Orbits and Attractors of $J_{3}^{\mathbb{Q}}$ and $J_{3}^{\mathbb{H}}$}

Let us now apply the above analysis to the $N=2, d=4$ "magical" MESGTs, based on the symmetric special Kähler manifolds

$$
\frac{E_{7(-25)}}{E_{6} \otimes U(1)}, \quad \frac{S O^{*}(12)}{U(6)} .
$$

defined by simple Jordan algebras $J_{3}^{\mathbb{Q}}$ and $J_{3}^{\mathbb{H}}$ of Hermitian $3 \times 3$ matrices over octonions (1) and quaternions $\mathbb{H}$, respectively. 
4.3.1 $N=2,6$ SUGRAs and the dual role of $\frac{S O^{*}(12)}{U(6)}$

Before proceeding further, also in order to stress the relevance of such two "magical" MESGTs, it is here worth pointing out some similarities and differences with respect to $N=8, d=4$ SUGRA, based on $\frac{E_{7(7)}}{S U(8)}$ and treated in Sect. 11.

Since the duality groups of $N=8, d=4$ SUGRA and $N=2, d=4$ "magical" $\frac{E_{7(-25)}}{E_{6} \otimes U(1)}$-based MESGT are two different real (non-compact) forms of the Lie exceptional group $E_{7} \equiv E_{7(-133)}\left(E_{7(7)}\right.$ and $E_{7(-25)}$, respectively), both their "charge vectors" are in the real symplectic representation ${ }^{14} 56$ of $E_{7}$.

Nevertheless, while the real (non-compact) form $E_{7(7)}$ contains only two different forms of $E_{6}$, namely the real (non-compact) forms $E_{6(2)}$ and $E_{6(6)}$, the group $E_{7(-25)}$ contains three different forms of $E_{6}: E_{6} \equiv E_{6(-78)}$ and its real (non-compact) forms $E_{6(-14)}$ and $E_{6(-26)}$. This fact is of course related to the fundamental difference that in $N=8$, $d=4 \frac{E_{7(7)}}{S U(8)}$-based SUGRA there are only two classes of 55-dim. non-degenerate orbits, whereas all $N=2, d=4$ MESGTs with symmetric scalar manifolds that originate from 5 dimensions, in particular the "exceptional" MESGT with the scalar manifold $\frac{E_{7(-25)}}{E_{6} \otimes U(1)}$, admit three distinct classes of such orbits.

By recalling the criticality conditions (2.12) and (2.17) for $V_{B H}$, also previously referred to as the $N=2, d=4$ extremal black hole attractor eqs., let us stress once again that the aforementioned three classes of $N=2$ non-degenerate orbits correspond to the following three classes of attractor solutions:

$\frac{1}{2}$-BPS : $Z \neq 0, D_{i} Z=0, \forall i=1, \ldots, n_{V}$;

(non-BPS $)_{\mathbf{1}}: Z \neq 0, D_{i} Z \neq 0$, for some $i \in\left\{1, \ldots, n_{V}\right\}$;

(non-BPS $)_{2}: Z=0, D_{i} Z \neq 0$, for some $i \in\left\{1, \ldots, n_{V}\right\}$.

Notice that the class (non-BPS) ${ }_{\mathbf{2}}$, corresponding to non-degenerate orbits of the class $\mathcal{O}_{n o n-B P S, Z=0}$, does not exist in $N=8, d=4 \frac{E_{7(7)}}{S U(8)}$-based SUGRA.

As it can be explicitly computed in the manageable yet interestingly rich case of the stu model [69], the classes $\frac{1}{2}$-BPS and (non-BPS) ${ }_{2}$ of solutions, and correspondingly the classes $\mathcal{O}_{\frac{1}{2}-B P S}$ and $\mathcal{O}_{n o n-B P S, Z=0}$ of orbits, have a strictly positive quartic $E_{7}$-invariant $I_{4}>0$. On the other hand, the class (non-BPS) ${ }_{1}$ of solutions, and thus the class $\mathcal{O}_{n o n-B P S, Z \neq 0}$ of orbits, have a strictly negative quartic $E_{7}$-invariant $I_{4}<0$.

At this point, we notice that both real (non-compact) forms of $E_{7}$, namely $E_{7(7)}$ of Cremmer and Julia [28] and $E_{7(-25)}$ of GST [37] contain the factorized group $S O^{*}(12) \otimes$ $S U(2)$ as a subgroup, and indeed both manifolds $\frac{E_{7(-25)}}{E_{6} \otimes U(1)}\left(\operatorname{dim}_{\mathbb{R}}=54\right)$ and $\frac{E_{7(7)}}{S U(8)}\left(\operatorname{dim}_{\mathbb{R}}=\right.$ 70) contain as a submanifold the coset space $\frac{S O^{*}(12)}{U(6)}$, which is the symmetric special

\footnotetext{
${ }^{14}$ As mentioned in Sect. 11 for $E_{7}$ the real symplectic and the fundamental representations coincide, but this is not generally the case.
} 
Kähler manifold of the $N=2, d=4$ "magical" MESGT defined by the Jordan algebra $J_{3}^{\mathbb{H}}[37$.

Such an observation reveals the dual role of the manifold $\frac{S O^{*}(12)}{U(6)}$ : it is at the same time the $\sigma$-model part of an $N=6$ SUGRA (if one starts from $N=8$, i.e. from $E_{7(7)}$ ) and of an $N=2$ MESGT (if one starts from $N=2$, i.e. from $E_{7(-25)}$ ).

The truncation from $N=8$ starts from the following decomposition of the real representation $R_{H}=\mathbf{7 0}$ of the $\mathcal{R}$-symmetry group $H=S U(8)$ in terms of representations of $S U(6) \otimes S U(2)$ [37, 31]:

$$
70 \longrightarrow(15,1)+(\overline{15}, \overline{1})+(20,2) .
$$

Under such a splitting the $70 N=8$ real scalars decompose in 15 complex scalars belonging to the submanifold $\frac{S O^{*}(12)}{U(6)} \varsubsetneqq \frac{E_{7(7)}}{S U(8)}$ and in 20 (half-)hypermultiplet scalars belonging to the submanifold $\frac{E_{6(2)}}{S U(6) \otimes S U(2)} \varsubsetneqq \frac{E_{7(7)}}{S U(8)}$. Consequently, the $\frac{S O^{*}(12)}{U(6)}$-based model is then reached by disregarding all the $S U(2)$-non-singlet terms in r.h.s. of decomposition (4.3.1.1), namely $(\mathbf{2 0}, \mathbf{2})$. Such a procedure eliminates two gravitinos from the SUGRA multiplet, and thus an $N=6, d=4 \frac{S O^{*}(12)}{U(6)}$-based SUGRA is obtained.

On the other hand, the truncation from the exceptional $N=2$ MESGT yields the following decomposition of the complex representation $R_{H_{0}}=\mathbf{2 7}$ of $H_{0}=E_{6}$ in terms of representations of its (non-maximal) compact subgroup $S U(6) \otimes S U(2)$ :

$$
27 \longrightarrow(15,1)+(6,2) .
$$

Once again, the $\frac{S O^{*}(12)}{U(6)}$-based model is reached by disregarding all the $S U(2)$-non-singlet terms in r.h.s. of decomposition (4.3.1.2), namely $(\mathbf{6}, \mathbf{2})$. In this case, the elimination of $S U(2)$-non-singlets does not touch the two gravitinos of the SUGRA multiplet, and thus an $N=2, d=4, \frac{S O^{*}(12)}{U(6)}$-based SUGRA is obtained, namely the "magical" MESGT defined by $J_{3}^{\mathbb{H}}$ [37].

Thus, the $N=2$ and $N=6 d=4$ SUGRAs with scalar manifold $\frac{S O^{*}(12)}{U(6)}$ have indistinguishable full bosonic sectors, and therefore their charge orbits are the same and their attractor Eqs. have the same solutions. Since we discussed above that an $N=2$, $d=4$ MESGT generally yield three distinct classes of non-degenerate orbits, the same must hold for the considered $N=6$ SUGRA.

This actually holds true, because the "charge vector" $\mathcal{C}$ of the $N=6, d=4 \frac{S O^{*}(12)}{U(6)}$ based theory, although it is a pure SUGRA, contains a vector which is a singlet under $S U(6), U(6)$ being the $\mathcal{R}$-symmetry [70]. Thus, the whole $N=6$ "charge vector" can be written as

$$
\mathcal{C}=\left(X, \widehat{Z}_{A B}\right)
$$

where $X$ is the $S U(6)$-singlet "charge vector" and $\widehat{Z}_{A B}(A=1, \ldots, N=6)$ is the complex $6 \times 6$ antisymmetric central charge matrix. 
Correspondingly, $N=6, d=4 \frac{S O^{*}(12)}{U(6)}$-based SUGRA has three distinct classes of extremal black hole solutions with finite, non-vanishing entropy, corresponding to the following structures of $\mathcal{C}$ [70]:

$X=0, \widehat{Z}_{A B} \neq 0$ (precisely $\widehat{Z}_{12} \neq 0, \widehat{Z}_{34}=0, \widehat{Z}_{56}=0$ in the "normal" frame).

This solution is a $\frac{1}{6}$-BPS $N=6$ solution, and it has

$$
I_{4, \frac{1}{6}-B P S}=|Z|_{\frac{1}{6}-B P S}^{4}>0
$$

$X \neq 0, \widehat{Z}_{A B}=0$.

Such a case corresponds to an $N=6$ non-BPS solution (since $\widehat{Z}_{A B}=0$ ), but, due to the contribution of $X$, it has

$$
I_{4, n o n-B P S, \widehat{Z}_{A B}=0}=|X|_{n o n-B P S, \widehat{Z}_{A B}=0}^{4}>0 .
$$

$X \neq 0, \widehat{Z}_{A B} \neq 0$ (precisely $|X|=\left|\widehat{Z}_{12}\right|=\left|\widehat{Z}_{34}\right|=\left|\widehat{Z}_{56}\right|$ in the "normal" frame).

Such a case corresponds to an $N=6$ non-BPS solution with

$$
I_{4, n o n-B P S, \widehat{Z}_{A B} \neq 0}=-16|X|_{n o n-B P S, \widehat{Z}_{A B} \neq 0}^{4}<0 \text {. }
$$

At a generic point of the scalar manifold $\frac{S O^{*}(12)}{U(6)}$ the overall symmetry of the modulidependent matrix $\widehat{Z}_{A B}$, which can be put in the skew-diagonal form $\widehat{Z}_{A B, \text { normal }}$, is $(S U(2))^{\frac{N}{2}=3}$. Thus, by considering the aforementioned structures of $\widehat{Z}_{A B}$ at regular solutions, one can determine the overall symmetry and the coset expression of the corresponding classes of non-degenerate orbits:

$X=0, \frac{1}{6}$-BPS solution: overall symmetry $S U(2) \otimes S U(4)$. Consequently, the unique choice for the coset expression of the class of non-degenerate $N=6 \frac{1}{6}$-BPS orbits is $(S U(2) \otimes S U(4) \otimes U(1)=$ m.c.s. $(S U(4,2)))$

$$
\mathcal{O}_{\frac{1}{6}-B P S}=\frac{S O^{*}(12)}{S U(4,2)} .
$$

$X \neq 0$, non-BPS, $\widehat{Z}_{A B}=0$ solution: overall symmetry $S U(6)$ :

$$
\mathcal{O}_{n o n-B P S, \widehat{Z}_{A B}=0}=\frac{S O^{*}(12)}{S U(6)} .
$$

Notice that such a class of $N=6$ solutions does not have an $N=8$ analogue; it is peculiar to $N=6 \mathrm{SUGRA}$, due to the particular form (4.3.1.3) of the "charge vector" $\mathcal{C}$. 


\begin{tabular}{|c||c|c|}
\hline Orbit & $N=6$ & $N=2$ \\
\hline \hline$\frac{S O^{*}(12)}{S U(6)}$ & $\mathcal{O}_{n o n-B P S, \widehat{Z}_{A B}=0}:\left\{\begin{array}{l}X \neq 0, \\
\widehat{Z}_{A B}=0, \\
\forall(A, B) \in\{1, \ldots, 6\}^{2}\end{array}\right.$ & $\mathcal{O}_{\frac{1}{2}-B P S}:\left\{\begin{array}{l}Z \neq 0, \\
D_{i} Z=0, \\
\forall i=1, \ldots, n_{V}=15\end{array}\right.$ \\
\hline$\frac{S O^{*}(12)}{S U(4,2)}$ & $\mathcal{O}_{\frac{1}{6}-B P S}:\left\{\begin{array}{l}X=0 \\
\widehat{Z}_{A B} \neq 0\end{array}\right.$ & $\mathcal{O}_{\text {non-BPS,Z=0 }}:\left\{\begin{array}{c}Z=0 \\
D_{i} Z \neq 0\end{array}\right.$ \\
\hline$\frac{S O^{*}(12)}{S U^{*}(6)}$ & $\mathcal{O}_{n o n-B P S, \widehat{Z}_{A B} \neq 0}:\left\{\begin{array}{c}X \neq 0 \\
\widehat{Z}_{A B} \neq 0\end{array}\right.$ & $\mathcal{O}_{\text {non-BPS, } Z \neq 0}:\left\{\begin{array}{c}Z \neq 0 \\
D_{i} Z \neq 0\end{array}\right.$ \\
\hline
\end{tabular}

Table 9: $N$-dependent BPS-interpretations of the classes of non-degenerate orbits of the homogeneous symmetric special Kähler manifold $\frac{S O^{*}(12)}{U(6)} . \widehat{Z}_{A B} \neq 0$ and $D_{i} Z \neq 0$ are generally understood for some values of $(A, B) \in\{1, \ldots, 6\}^{2}$ and of $i \in\left\{1, \ldots, n_{V}=15\right\}$, respectively.

$X \neq 0$, non-BPS, $\widehat{Z}_{A B} \neq 0$ solution: overall symmetry is $U S p(6)$ and the corresponding orbit reads $\left(U S p(6)=\right.$ m.c.s. $\left.\left(S U^{*}(6)\right)\right)$

$$
\mathcal{O}_{n o n-B P S, \widehat{Z}_{A B} \neq 0}=\frac{S O^{*}(12)}{S U^{*}(6)} .
$$

It is now immediate to notice that the classes of non-degenerate orbits of the considered $N=6, d=4$ case are the same of those of the $N=2, d=4$ "magical" MESGT based on $\frac{S O^{*}(12)}{U(6)}$, but with different BPS features. The $N$-dependent BPS-interpretations of the classes of non-degenerate orbits of the irreducible homogeneous symmetric special Kähler manifold $\frac{S O^{*}(12)}{U(6)}$ are summarized in Table 9.

As it can be seen from Table 9, the role of regular BPS orbits and non-BPS orbits with (all) central charge(s) vanishing is flipped under the exchange $N=2 \longleftrightarrow N=6$. Such a kind of "cross-symmetry" is easily understood when noticing that the $N=2$ central charge $Z$ corresponds to the $S U(6)$-singlet component $X$ of the $N=6$ "charge vector" $\mathcal{C}$, and that the 15 complex $N=2$ "matter charges" $D_{i} Z$ correspond to the 15 independent complex elements of the $6 \times 6$ antisymmetric $N=6$ central charge matrix $\widehat{Z}_{A B}$.

Also, Table 9 immediately yields another consequence of the " $N=2 \longleftrightarrow N=6$ cross-symmetry": while in all $N=2, d=4$ irreducible symmetric MESGTs the class of regular BPS critical points is more symmetric than both classes of regular non-BPS critical points, a different result holds for the $N=6, d=4 \frac{S O^{*}(12)}{U(6)}$-based SUGRA: the 
most symmetric regular solutions are the non-BPS ones with $\widehat{Z}_{A B}=0$ and $X \neq 0$, and not the $\frac{1}{6}-B P S$ ones.

\subsubsection{Orbits and Attractors of $J_{3}^{\mathbb{Q}}$}

Let us now apply the general analysis performed in Sect. 4 to the case of the exceptional $N=2, d=4$ MESGT with scalar manifold $\frac{E_{7(-25)}}{E_{6} \otimes U(1)}$, defined by the exceptional Jordan algebra $J_{3}^{\mathbb{Q}}$. As already mentioned above, this is the MESGT with the largest scalar manifold (apart from the two sequences $I$ and $I I$ ), and it is the only "magical" MESGT which is not a consistent truncation of $N=8, d=4 \frac{E_{7(7)}}{S U(8)}$-based SUGRA [37]. In this case

$$
G=E_{7(-25)}, H_{0}=E_{6}=\frac{m . c . s .\left(E_{7(-25)}\right)}{U(1)}=\operatorname{Ktr}_{0}\left(J_{3}^{\mathbb{Q}}\right) \text {. }
$$

$\frac{1}{2}$-BPS solutions $\mathcal{O}_{\frac{1}{2}-B P S, J_{3}^{\oplus}}=\frac{E_{7(-25)}}{E_{6}}$

They are given by

$$
\begin{aligned}
& Z \neq 0, D_{i} Z=0 \Leftrightarrow D_{I} Z=0, \forall i, I=1, \ldots, n_{V}=27 \\
& V_{B H, \frac{1}{2}-B P S, J_{3}^{\oplus}}=|Z|_{\frac{1}{2}-B P S, J_{3}^{\oplus}}^{2}
\end{aligned}
$$

and they are manifestly $E_{6}$-invariant.

Non-BPS, $Z \neq 0$ solutions $\mathcal{O}_{n o n-B P S, Z \neq 0, J_{3}^{\oplus}}=\frac{E_{7(-25)}}{E_{6(-26)}}$

The non-compact stabilizer of the orbit is $\widehat{H}=E_{6(-26)}=\operatorname{Str}_{0}^{*}\left(J_{3}^{\mathbb{Q}}\right)$. The "flatted matter charges" $D_{I} Z$ sit in the complex representation $R_{H_{0}}=\mathbf{2 7}$ of $H_{0}=E_{6}$. Under $\widehat{h}=F_{4}=$ m.c.s. $\left(E_{6(-26)}\right), R_{H_{0}}$ decomposes as

$$
27 \longrightarrow 26+1
$$

where the r.h.s. is made of the complex representations $\mathbf{1}$ and $\mathbf{2 6}$ of $F_{4}$. Such a decomposition yields the following splitting of "flatted matter charges":

$$
D_{I} Z \longrightarrow\left(D_{26} Z, D_{1} Z\right)
$$

where the subscripts "26" and " $\mathbf{1}$ " denote the directions along the $\mathbf{2 6}$ and the $F_{4}$-singlet direction in $\frac{E_{7(-25)}}{E_{6} \otimes U(1)}$, respectively. By decomposing $\left(R_{H_{0}}\right)^{3}=(\mathbf{2 7})^{3}$ in terms of representations of $F_{4}$, one therefore finds

$$
(\mathbf{2 7})^{3} \longrightarrow(\mathbf{2 6})^{3}+(\mathbf{2 6})^{2} \mathbf{1}+\mathbf{1}^{3} \Leftrightarrow C_{I J K} \longrightarrow\left\{C_{\mathbf{2 6 , 2 6 , 2 6}}, C_{\mathbf{2 6 , 2 6 , 1}}, C_{\mathbf{1}, \mathbf{1}, \mathbf{1}}\right\} .
$$

Thus, the solution of $N=2, d=4$ non-BPS, $Z \neq 0$ extremal black hole attractor eqs. in "flat" indices (4.2.3) for the treated case is obtained by putting

$$
D_{26} Z=0, D_{1} Z \neq 0,
$$


constrained by

$$
2 \bar{Z} D_{\mathbf{1}} Z=-i C_{\mathbf{1 , 1}, \mathbf{1}}\left(\overline{D_{\mathbf{1}} Z}\right)^{2}
$$

where we recall that (see Eq. (4.2.1.7))

$$
\left|C_{\mathbf{1}, \mathbf{1}, \mathbf{1}}\right|_{\text {non }-B P S, Z \neq 0, J_{3}^{\mathbb{Q}}}^{2}=\frac{4}{3} .
$$

The resulting value of the black hole scalar potential at the considered attractor point $(\mathrm{s})$ is

$$
\begin{aligned}
& V_{B H, n o n-B P S, Z \neq 0, J_{3}^{\oplus}}=|Z|_{n o n-B P S, Z \neq 0, J_{3}^{\mathbb{Q}}}^{2}+\left[G^{i \bar{i}} D_{i} Z \bar{D} \overline{\bar{i}} \bar{Z}\right]_{n o n-B P S, Z \neq 0, J_{3}^{\oplus}}=
\end{aligned}
$$

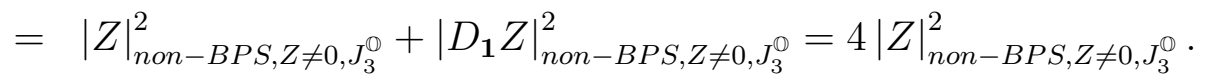

The overall symmetry group at $N=2$ non-BPS, $Z \neq 0$ critical point(s) of $J_{3}^{\mathbb{Q}}$ is $\widehat{h}=$ $F_{4}=$ m.c.s. $\left(E_{6(-26)}\right)$.

Non-BPS, $Z=0$ solutions $\mathcal{O}_{n o n-B P S, Z=0, J_{3}^{\mathbb{Q}}}=\frac{E_{7(-25)}}{E_{6(-14)}}$

The non-compact stabilizer of the orbit is $\widetilde{H}=E_{6(-14)}=\Delta_{0}\left(J_{3}^{\mathbb{D}}\right)$. Under $\widetilde{h}^{\prime}=$ $S O(10)=\frac{m . c . s .\left(E_{6(-14)}\right)}{U(1)}, R_{H_{0}}=\mathbf{2 7}$ decomposes as

$$
27 \longrightarrow 16_{1}+10_{-2}+1_{4}
$$

where $\mathcal{W}_{\widetilde{h}^{\prime}}=\mathbf{1 6} \mathbf{6}_{1}, \mathcal{Y}_{\widetilde{h}^{\prime}}=\mathbf{1 0}_{-2}$ and $\mathbf{1}_{4}$ are complex $(U(1)$-charged) representations of $S O(10)$, and here the numeric subscripts denote the charges with respect to the explicit factor $U(1)$ in $\widetilde{h}=S O(10) \otimes U(1)=$ m.c.s. $\left(E_{6(-14)}\right)$.

The decomposition (4.3.2.10) yields the following splitting of "flatted matter charges":

$$
D_{I} Z \longrightarrow\left(D_{16} Z, D_{10} Z, D_{1} Z\right) .
$$

Thence, by decomposing the $E_{6}$-invariant representation $\left(R_{H_{0}}\right)^{3}=(\mathbf{2 7})^{3}$ in terms of representations of $S O(10)$, one finds

$$
(27)^{3} \longrightarrow 16_{1} 16_{1} 10_{-2}+10_{-2} 10_{-2} 1_{4} \Leftrightarrow C_{I J K} \longrightarrow\left\{C_{16,16,10}, C_{10,10,1}\right\}
$$

Thus, the solution of $N=2, d=4$ non-BPS, $Z=0$ extremal black hole attractor eqs. in "flat" indices (4.2.4) for the treated case is obtained by putting

$$
D_{16} Z=0=D_{10} Z, D_{1} Z \neq 0
$$

The resulting value of the black hole scalar potential at the considered attractor point $(\mathrm{s})$ is

$$
V_{B H, n o n-B P S, Z=0, J_{3}^{\oplus}}=\left|D_{1} Z\right|_{n o n-B P S, Z=0, J_{3}^{\bigotimes}}^{2} .
$$


Attention should be paid to distinguish between the $D_{1} Z$ 's appearing in Eqs. (4.3.2.9) and (4.3.2.14), since they refer to different directions in $\frac{E_{7(-25)}}{E_{6} \otimes U(1)}$ : in Eq. (4.3.2.9) $D_{1} Z$ denotes the "flatted matter charge" along the complex $F_{4}$-singlet, whereas in (4.3.2.14) $D_{1} Z$ stands for the "flatted matter charge" along the complex (U(1)-charged) $S O(10)$ singlet.

The overall symmetry group at $N=2$ non-BPS, $Z=0$ critical point(s) of $J_{3}^{\mathbb{Q}}$ is $\widetilde{h}^{\prime}=S O(10)=\frac{\widetilde{h}}{U(1)}$, where $\widetilde{h}=S O(10) \otimes U(1)=$ m.c.s. $\left(E_{6(-14)}\right)$.

\subsubsection{Orbits and Attractors of $J_{3}^{\mathbb{H}}$}

Let us now move to consider the case of the $N=2, d=4$ symmetric "magical" MESGT with scalar manifold $\frac{S O^{*}(12)}{U(6)}$, related to $J_{3}^{\mathbb{H}}$.

This is the "magical" MESGT with the largest scalar manifold which can be obtained as a consistent truncation of $N=8, d=4 \frac{E_{7(7)}}{S U(8)}$-based SUGRA. The other two $N=2$, $d=4$ symmetric "magical" MESGTs with a smaller scalar manifold (namely those based on $\frac{S U(3,3)}{S U(3) \otimes S U(3) \otimes U(1)}$ and $\frac{S p(6, \mathbb{R})}{U(3)}$ : see Tables 2 and 3) can actually be obtained as consistent truncations of such a $\frac{S O^{*}(12)}{U(6)}$-based "magical" MESGT.

Moreover, as pointed out in Subsubsect. 4.3.1, it plays a dual role, since $\frac{S O^{*}(12)}{U(6)}$ is also the scalar manifold of $N=6, d=4$ SUGRA. It is now worth analyzing the same classes of non-degenerate charge orbits as in Subsubsect. 4.3.1 with an $N=2, d=4$ approach based on special Kähler geometry.

In this case

$$
G=S O^{*}(12), H_{0}=S U(6)=\frac{m . c . s .\left(S O^{*}(12)\right)}{U(1)}=K \operatorname{tr}_{0}\left(J_{3}^{\mathbb{H}}\right) .
$$

$\frac{1}{2}$-BPS solutions $\mathcal{O}_{\frac{1}{2}-B P S, J_{3}^{\mathbb{H}}}=\frac{S O^{*}(12)}{S U(6)}$

They are given by

$$
\begin{aligned}
& Z \neq 0, D_{i} Z=0 \Leftrightarrow D_{I} Z=0, \forall i, I=1, \ldots, n_{V}=15 ; \\
& V_{B H, \frac{1}{2}-B P S, J_{3}^{\mathbb{H}}}=|Z|_{\frac{1}{2}-B P S, J_{3}^{\mathbb{H}}}^{2},
\end{aligned}
$$

and they are manifestly $S U(6)$-invariant.

Non-BPS, $Z \neq 0$ solutions $\mathcal{O}_{n o n-B P S, Z \neq 0, J_{3}^{\mathbb{H}}}=\frac{S O^{*}(12)}{S U^{*}(6)}$

The non-compact stabilizer of the orbit is $\widehat{H}=S U^{*}(6)=\operatorname{Str}_{0}^{*}\left(J_{3}^{\mathbb{H}}\right)$. The "flatted matter charges" $D_{I} Z$ sit in the complex representation $R_{H_{0}}=\mathbf{1 5}$ of $H_{0}$; under $\widehat{h}=$ $U S p(6)=$ m.c.s. $\left(S U^{*}(6)\right), R_{H_{0}}$ decomposes as

$$
15 \longrightarrow 14+1
$$


where the r.h.s. is made of the complex representations $\mathbf{1}$ and $\mathbf{1 4}$ of $U S p(6)$. Such a decomposition yields the following splitting of "flatted matter charges":

$$
D_{I} Z \longrightarrow\left(D_{14} Z, D_{1} Z\right) \text {. }
$$

By decomposing $\left(R_{H_{0}}\right)^{3}=(\mathbf{1 5})^{3}$ in terms of representations of $U S p(6)$, one therefore finds

$$
(\mathbf{1 5})^{3} \longrightarrow(\mathbf{1 4})^{3}+(\mathbf{1 4})^{2} \mathbf{1}+\mathbf{1}^{3} \Leftrightarrow C_{I J K} \longrightarrow\left\{C_{\mathbf{1 5}, \mathbf{1 5}, \mathbf{1 5}}, C_{\mathbf{1 5}, \mathbf{1 5}, \mathbf{1}}, C_{\mathbf{1 , 1 , 1}}\right\} .
$$

Thus, the solution of $N=2, d=4$ non-BPS, $Z \neq 0$ extremal black hole attractor eqs. in "flat" indices (4.2.3) for the treated case is obtained by putting

$$
D_{14} Z=0, D_{1} Z \neq 0,
$$

constrained by Eq. (4.3.2.7), once again with $\left|C_{\mathbf{1}, \mathbf{1}, \mathbf{1}}\right|_{\text {non- }-B P S, Z \neq 0, J_{3}^{\mathbb{H}}}^{2}=\frac{4}{3}$.

The resulting value of the black hole scalar potential at the considered attractor point(s) is once again

$$
\begin{aligned}
& V_{B H, n o n-B P S, Z \neq 0, J_{3}^{\mathbb{H}}}= \\
& =|Z|_{n o n-B P S, Z \neq 0, J_{3}^{\mathbb{H}}}^{2}+\left|D_{1} Z\right|_{n o n-B P S, Z \neq 0, J_{3}^{\mathbb{H}}}^{2}=4|Z|_{n o n-B P S, Z \neq 0, J_{3}^{\mathbb{H}}}^{2} .
\end{aligned}
$$

The overall symmetry group at $N=2$ non-BPS, $Z \neq 0$ critical point(s) of $J_{3}^{\mathbb{H}}$ is $\widehat{h}=$ $U S p(6)=$ m.c.s. $\left(S U^{*}(6)=\operatorname{Str}_{0}^{*}\left(J_{3}^{\mathbb{H}}\right)\right.$.

Non-BPS, $Z=0$ solutions $\mathcal{O}_{n o n-B P S, Z=0, J_{3}^{\mathbb{H}}}=\frac{S O^{*}(12)}{S U(4,2)}$

The non-compact stabilizer of the orbit is $\widetilde{H}=S U(4,2)$. Under $\widetilde{h}^{\prime}=S U(4) \otimes S U(2)=$ $\frac{m . c . s .(S U(4,2))}{U(1)}, R_{H_{0}}=\mathbf{1 5}$ decomposes as

$$
15 \longrightarrow(4,2)_{-1}+(6,1)_{2}+(1,1)_{-4},
$$

where $\mathcal{W}_{\widetilde{h}^{\prime}}=(\mathbf{4}, \mathbf{2})_{-1}, \mathcal{Y}_{\widetilde{h}^{\prime}}=(\mathbf{6}, \mathbf{1})_{2}$ and $(\mathbf{1}, \mathbf{1})_{-4}$ are complex $(U(1)$-charged $)$ representations of $S U(4) \otimes S U(2)$, and as above the numeric subscripts denote the charges with respect to the explicit factor $U(1)$ in $\widetilde{h}=S U(4) \otimes S U(2) \otimes U(1)=$ m.c.s. $(S U(4,2))$.

The decomposition (4.3.3.8) yields the following splitting of "flatted matter charges":

$$
D_{I} Z \longrightarrow\left(D_{(\mathbf{4}, \mathbf{2})} Z, D_{(\mathbf{6 , 1})} Z, D_{(\mathbf{1}, \mathbf{1})} Z\right)
$$

Thence, by decomposing the $S U(6)$-invariant representation $\left(R_{H_{0}}\right)^{3}=(\mathbf{1 5})^{3}$ in terms of representations of $S U(4) \otimes S U(2)$, one finds

$$
\begin{gathered}
(\mathbf{1 5})^{3} \longrightarrow(\mathbf{4}, \mathbf{2})_{-1}(\mathbf{4}, \mathbf{2})_{-1}(\mathbf{6}, \mathbf{1})_{2}+(\mathbf{6}, \mathbf{1})_{2}(\mathbf{6}, \mathbf{1})_{2}(\mathbf{1}, \mathbf{1})_{-4} \\
\mathbb{1} \\
C_{I J K} \longrightarrow\left\{C_{(\mathbf{4}, \mathbf{2}),(\mathbf{4}, \mathbf{2}),(\mathbf{6}, \mathbf{1})}, C_{(\mathbf{6}, \mathbf{1}),(\mathbf{6}, \mathbf{1}),(\mathbf{1}, \mathbf{1})}\right\} .
\end{gathered}
$$


Thus, the solution of $N=2, d=4$ non-BPS, $Z=0$ extremal black hole attractor eqs. in "flat" indices (4.2.4) for the treated case is obtained by putting

$$
D_{(\mathbf{4}, \mathbf{2})} Z=0=D_{(\mathbf{6}, \mathbf{1})} Z, \quad D_{(\mathbf{1}, \mathbf{1})} Z \neq 0
$$

The resulting value of the black hole scalar potential at the considered attractor point $(\mathrm{s})$ is

$$
V_{B H, \text { non }-B P S, Z=0, J_{3}^{\mathbb{H}}}=\left|D_{(\mathbf{1}, \mathbf{1})} Z\right|_{\text {non- }-B P S, Z=0, J_{3}^{\mathbb{H}}}^{2} \cdot
$$

Once again, attention should be paid to distinguish between the $D_{1} Z$ 's appearing in Eqs. (4.3.3.7) and (4.3.3.13), since they refer to different directions in $\frac{S O^{*}(12)}{U(6)}$ : in Eq. (4.3.3.7) $D_{1} Z$ denotes the "flatted matter charge" along the complex USp(6)-singlet, whereas in (4.3.3.13) $D_{(\mathbf{1}, \mathbf{1})} Z$ stands for the "flatted matter charge" along the complex $(U(1)$-charged $)(S U(4) \otimes S U(2))$-singlet.

The overall symmetry group at $N=2$ non-BPS, $Z=0$ critical point(s) of $J_{3}^{\mathbb{H I}}$ is $\widetilde{h}^{\prime}=S U(4) \otimes S U(2)=\frac{\widetilde{h}}{U(1)}$, where $\widetilde{h}=S U(4) \otimes S U(2) \otimes U(1)=$ m.c.s. $(S U(4,2))$.

A completely analogous analysis may be performed for the cases $V\left(J_{3}^{\mathbb{C}}\right)$ and $V I\left(J_{3}^{\mathbb{R}}\right)$ of Tables 2 and 3. We leave such an analysis as an instructive exercise for the reader.

\section{The Mass Spectra at Critical Points}

The black hole scalar potential $V_{B H}$ gives different masses to the different BPS-phases of the considered symmetric $N=2, d=4$ MESGTs. The fundamental object to be considered in such a framework is the moduli-dependent $2 n_{V} \times 2 n_{V}$ Hessian matrix of $V_{B H}$, which in complex basis reads ${ }^{15}[54]$

$$
\begin{aligned}
& \mathbf{H}^{V_{B H}} \equiv\left(\begin{array}{cc}
D_{i} D_{j} V_{B H} & D_{i} \bar{D}_{\bar{j}} V_{B H} \\
D_{j} \bar{D}_{\bar{i}} V_{B H} & \bar{D}_{\bar{i}} \bar{D}_{\bar{j}} V_{B H}
\end{array}\right) \equiv\left(\begin{array}{cc}
\mathcal{M}_{i j} & \mathcal{N}_{i \bar{j}} \\
\overline{\mathcal{N}}_{j \bar{i}} & \overline{\mathcal{M}}_{\overline{i j}}
\end{array}\right) ; \\
& \mathcal{M}_{i j} \equiv D_{i} D_{j} V_{B H}=D_{j} D_{i} V_{B H}= \\
& =4 i \bar{Z} C_{i j k} G^{k \bar{k}} \bar{D}_{\bar{k}} \bar{Z}+i G^{k \bar{k}} G^{l \bar{l}} D_{j} C_{i k l} \bar{D}_{\bar{k}} \bar{Z} \bar{D}_{\bar{l}} \bar{Z} \\
& \mathcal{N}_{i \bar{j}} \equiv D_{i} \bar{D}_{\bar{j}} V_{B H}=\bar{D}_{\bar{j}} D_{i} V_{B H}= \\
& =2\left[G_{i \bar{j}}|Z|^{2}+D_{i} Z \bar{D}_{\bar{j}} \bar{Z}+G^{l \bar{n}} G^{k \bar{k}} G^{m \bar{m}} C_{i k l} \bar{C}_{\bar{j} \overline{m n}} \bar{D}_{\bar{k}} \bar{Z} D_{m} Z\right] \\
& \mathcal{M}^{T}=\mathcal{M}, \mathcal{N}^{\dagger}=\mathcal{N} .
\end{aligned}
$$

\footnotetext{
${ }^{15}$ The reported formulæ for $\mathcal{M}_{i j}$ and $\mathcal{N}_{i \bar{j}}$ hold for any special Kähler manifold. In the symmetric case formula (5.2) gets simplified using Eq. (2.14).
} 
By analyzing $\mathbf{H}^{V_{B H}}$ at regular critical points, it is possible to formulate general conclusions about the mass spectrum of the corresponding extremal black hole solutions with finite, non-vanishing entropy, i.e. about the mass spectrum along the related classes of nondegenerate charge orbits of the symplectic real representation $R_{V}$ of the $d=4$ duality group $G$.

Let us start by remarking that, due to its very definition (2.11), the $N=2$ black hole scalar potential $V_{B H}$ is positive for any (not necessarily strictly) positive definite metric $G_{i \bar{i}}$ of the scalar manifold. Consequently, the stable critical points (i.e. the attractors in a strict sense) will necessarily be minima of such a potential. As already pointed out above and as done also in [54, 55], the geometry of the scalar manifold is usually assumed to be regular, i.e. endowed with a metric tensor $G_{i \bar{j}}$ being strictly positive definite everywhere.

\section{$\frac{1}{2}$-BPS critical points}

It is now well known that regular special Kähler geometry implies that all $N=2$ $\frac{1}{2}$-BPS critical points of all $N=2, d=4$ MESGTs are stable, and therefore they are attractors in a strict sense. Indeed, the Hessian matrix $\mathbf{H}_{\frac{1}{2}-B P S}^{V_{B H}}$ evaluated at such points is strictly positive definite [14]:

$$
\begin{aligned}
& \mathcal{M}_{i j, \frac{1}{2}-B P S}=0, \\
& \mathcal{N}_{i \bar{j}, \frac{1}{2}-B P S}=\left.2 G_{i \bar{j}}\right|_{\frac{1}{2}-B P S}|Z|_{\frac{1}{2}-B P S}^{2}>0,
\end{aligned}
$$

where the notation " $>0$ " is clearly understood as strict positive definiteness of the quadratic form related to the square matrix being considered. Notice that the Hermiticity and strict positive definiteness of $\mathbf{H}_{\frac{1}{2}-B P S}^{V_{B H}}$ are respectively due to the Hermiticity and strict positive definiteness of the Kähler metric $G_{i \bar{j}}$ of the scalar manifold.

By switching from the non-flat $i$-coordinates to the "flat" local $I$-coordinates by using the (inverse) Vielbein $e_{I}^{i}$ of the scalar manifold, Eqs. (5.5) can be rewritten as

$$
\begin{aligned}
& \mathcal{M}_{I J, \frac{1}{2}-B P S}=0, \\
& \mathcal{N}_{I \bar{J}, \frac{1}{2}-B P S}=2 \delta_{I \bar{J}}|Z|_{\frac{1}{2}-B P S}^{2}>0 .
\end{aligned}
$$

Thus, one obtains that in all $N=2, d=4$ MESGTs the $\frac{1}{2}$-BPS mass spectrum in "flat" coordinates is monochromatic, i.e. that all "particles" (i.e. the "modes" related to the degrees of freedom described by the "flat" local $I$-coordinates) acquire the same mass at $\frac{1}{2}$-BPS critical points of $V_{B H}$.

\section{Non-BPS, $Z \neq 0$ critical points}

In this case the result of Tripathy and Trivedi [17] should apply, namely the Hessian matrix $\mathbf{H}_{n o n-B P S, Z \neq 0}^{V_{B H}}$ should have $n_{V}+1$ strictly positive and $n_{V}-1$ vanishing real eigenvalues. 
By recalling the analysis performed in Sect. 4, it is thence clear that such massive and massless non-BPS, $Z \neq 0$ "modes" fit distinct real representations of $\widehat{h}=$ m.c.s. $(\widehat{H})$, where $\widehat{H}$ is the non-compact stabilizer of the class $\mathcal{O}_{n o n-B P S, Z \neq 0}=\frac{G}{\widehat{H}}$ of non-BPS, $Z \neq 0$ non-degenerate charge orbits.

This is perfectly consistent with the decomposition (4.2.1.1) of the complex representation $R_{H_{0}}\left(\operatorname{dim}_{\mathbb{R}} R_{H_{0}}=2 n_{V}\right)$ of $H_{0}$ in terms of representations of $\widehat{h}$ :

$$
R_{H_{0}} \longrightarrow\left(R_{\widehat{h}}+\mathbf{1}\right)_{\mathbb{C}}=\left(R_{\widehat{h}}+\mathbf{1}+R_{\widehat{h}}+\mathbf{1}\right)_{\mathbb{R}}, \operatorname{dim}_{\mathbb{R}}\left(R_{\widehat{h}}\right)_{\mathbb{R}}=n_{V}-1 .
$$

As yielded by the treatment given in Subsubsect. 4.2.1 the notation " $\left(R_{\widehat{h}}+\mathbf{1}\right)_{\mathbb{C}}=$ $\left(R_{\widehat{h}}+\mathbf{1}+R_{\widehat{h}}+\mathbf{1}\right)_{\mathbb{R}}$ " denotes nothing but the decomplexification of $\left(R_{\widehat{h}}+\mathbf{1}\right)_{\mathbb{C}}$, which is actually composed by a pair of real irreducible representations $\left(R_{\widehat{h}}+\mathbf{1}\right)_{\mathbb{R}}$ of $\widehat{h}$.

Therefore, Tripathy and Trivedi's result can be understood in terms of real representations of the m.c.s. of the non-compact stabilizer of $\mathcal{O}_{n o n-B P S, Z \neq 0}$ : the $n_{V}-1$ massless non-BPS, $Z \neq 0$ "modes" are in one of the two real $R_{\widehat{h}}$ 's of $\widehat{h}$ in the r.h.s. of Eq. (5.7), say the first one, whereas the $n_{V}+1$ massive non-BPS, $Z \neq 0$ "modes" are split in the remaining real $R_{\widehat{h}}$ of $\widehat{h}$ and in the two real $\widehat{h}$-singlets. The resulting interpretation of the decomposition (5.7) is

$$
R_{H_{0}} \longrightarrow\left(\begin{array}{c}
\left(R_{\widehat{h}}\right)_{\mathbb{R}} \\
n_{V}-1 \text { massless }
\end{array}\right)+\left(\begin{array}{c}
\left(R_{\widehat{h}}\right)_{\mathbb{R}}+\mathbf{1}_{\mathbb{R}}+\mathbf{1}_{\mathbb{R}} \\
n_{V}+1 \text { massive }
\end{array}\right) .
$$

It is interesting to notice once again that there is no $U(1)$ symmetry relating the two real

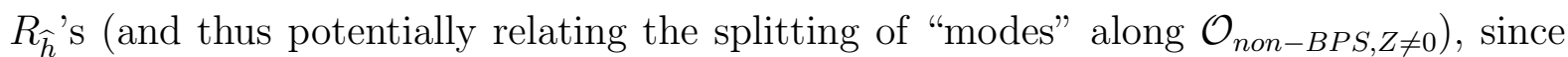
in all symmetric $N=2, d=4$ MESGTs $\widehat{h}$ never contains an explicit factor $U(1)$ (as instead it always happens for $\widetilde{h}$ !); this can be related to the fact that the non-compact stabilizer is $\widehat{H}=\operatorname{Str}_{0}^{*}(\mathcal{J})$ whose $\widehat{h}$ is the m.c.s..

For further elucidation, let us consider the explicit example of the $J_{3}^{\mathbb{Q}}$-related symmetric $N=2, d=4$ "magical" MESGT with scalar manifold $\frac{E_{7(-25)}}{E_{6} \otimes U(1)}\left(n_{V}=27\right)$, treated in Subsubsect. 4.3.2. In the sense of aforementioned decomplexification, the decomposition (4.3.2.3) of the complex representation $R_{H_{0}}=\mathbf{2 7}$ under $\widehat{h}=F_{4}$ can also be written as

$$
27 \longrightarrow 26+1=26_{\mathbb{R}}+\mathbf{1}_{\mathbb{R}}+26_{\mathbb{R}}+\mathbf{1}_{\mathbb{R}} \text {. }
$$

It is then clear that the mass spectrum of $\mathcal{O}_{n o n-B P S, Z \neq 0, J_{3}^{\oplus}}$ splits under $F_{4}$ as follows: the 26 massless non-BPS, $Z \neq 0$ "modes" are in one of the two $\mathbf{2 6}_{\mathbb{R}}$ 's of $F_{4}$ in the r.h.s. of Eq. (5.9), say the first one, whereas the 28 massive non-BPS, $Z \neq 0$ "modes" are split in the remaining $\mathbf{2 6}_{\mathbb{R}}$ of $F_{4}$ and in the two real $F_{4}$-singlets. The resulting interpretation of the decomposition (5.9) is

$$
\mathbf{2 7} \longrightarrow\left(\begin{array}{c}
\mathbf{2 6}_{\mathbb{R}} \\
26 \text { massless }
\end{array}\right)+\left(\begin{array}{c}
\mathbf{2 6}_{\mathbb{R}}+\mathbf{1}_{\mathbb{R}}+\mathbf{1}_{\mathbb{R}} \\
28 \text { massive }
\end{array}\right) .
$$




\section{Non-BPS, $Z=0$ critical points}

For the class $\mathcal{O}_{\text {non-BPS,Z=0 }}$ of non-degenerate non-BPS, $Z=0$ orbits the situation changes, and Tripathy and Trivedi's result no longer holds true, due to the local vanishing of the $N=2$ central charge.

In order to illustrate the consequences of $Z=0$ along $\mathcal{O}_{\text {non-BPS,Z=0 }}$ on the related mass spectrum, let us consider again the symmetric $N=2, d=4$ "magical" MESGT with scalar manifold $\frac{E_{7(-25)}}{E_{6} \otimes U(1)}$.

In such a case, $\mathcal{O}_{n o n-B P S, Z=0, J_{3}^{\oplus}}=\frac{E_{7(-25)}}{E_{6(-14)}}$, and therefore the non-compact stabilizer is $\widetilde{H}=E_{6(-14)}$. The complex 27 and the $E_{6}$-invariant $(\mathbf{2 7})^{3}$ of $E_{6}$ decompose under $\widetilde{h}^{\prime}=S O(10)=\frac{m . c . s .\left(E_{6(-14)}\right)}{U(1)}$ as given by Eqs. (4.3.2.10) and (4.3.2.12), respectively:

$$
\begin{gathered}
27 \longrightarrow 16_{1}+10_{-2}+1_{4} ; \\
(27)^{3} \longrightarrow 16_{1} 16_{1} 10_{-2}+10_{-2} 10_{-2} 1_{4} .
\end{gathered}
$$

Consequently, the rank-3 $E_{6}$-invariant tensor coupling $C_{I J K}$ decomposes in its nonvanishing components as follows:

$$
C_{I J K} \longrightarrow\left\{C_{16,16,10}, C_{10,10,1}\right\}
$$

In Subsubsect. 4.3.2 the solution of $N=2, d=4$ non-BPS, $Z=0$ extremal black hole attractor eqs. in "flat" indices (4.2.4) for the case at hand was found to be given by Eq. (4.3.2.13):

$$
D_{16} Z=0=D_{10} Z, D_{1} Z \neq 0 .
$$

By using Eqs. (5.13) and (5.14), the block matrix components of the $54 \times 54$ critical Hessian $\mathbf{H}_{n o n-B P S, Z=0, J_{3}^{\oplus}}^{V_{B H}}$ in "flat" coordinates can be computed to be:

$$
\begin{aligned}
& \mathcal{M}_{I J, n o n-B P S, Z=0, J_{3}^{\oplus}}=0 ; \\
& \mathcal{N}_{I \bar{J}, n o n-B P S, Z=0, J_{3}^{0}}= \\
& =2\left[D_{I} Z \bar{D} \bar{J} \bar{Z}+\delta^{L \bar{N}} \delta^{K \bar{K}} \delta^{M \bar{M}} C_{I K L} \bar{C} \overline{J M N} \bar{D}_{\bar{K}} \bar{Z} D_{M} Z\right]_{n o n-B P S, Z=0, J_{3}^{\infty}} .
\end{aligned}
$$

The only non-vanishing elements of the $27 \times 27$ critical (diagonal and real) matrix

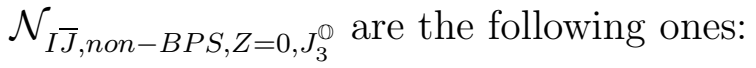

$$
\begin{aligned}
& \mathcal{N}_{\mathbf{1} \overline{1}, \text { non- } B P S, Z=0, J_{3}^{\oplus}}=2\left|D_{\mathbf{1}} Z\right|_{\text {non- }-B P S, Z=0, J_{3}^{\oplus}}^{2} ; \\
& \mathcal{N}_{\mathbf{1 0} \overline{\mathbf{1 0}}, \text { non- }-B P S, Z=0, J_{3}^{0}}=2\left|C_{\mathbf{1 0}, \mathbf{1 0}, \mathbf{1}}\right|_{\text {non }-B P S, Z=0, J_{3}^{\oplus}}^{2}\left|D_{\mathbf{1}} Z\right|_{\text {non }-B P S, Z=0, J_{3}^{\infty}}^{2},
\end{aligned}
$$


and therefore one gets (the subscripts denote the matrix dimension)

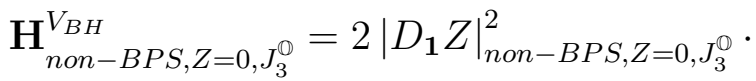

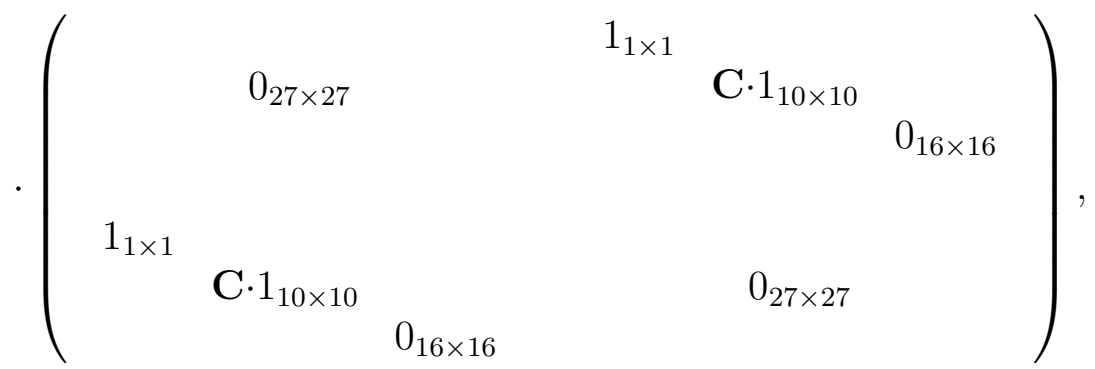

where $\mathbf{C} \equiv\left|C_{\mathbf{1 0 , 1 0 , 1}}\right|_{\text {non-BPS,Z=0,J3 }}^{2} \in \mathbb{R}_{0}^{+}$. The real form [54] of the non-BPS $Z=0$ critical Hessian finally reads

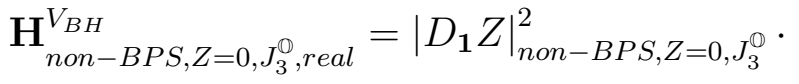

$$
\begin{aligned}
& \left(\begin{array}{cccccc}
1_{1 \times 1} & & & & & \\
& \mathbf{C} \cdot 1_{10 \times 10} & & & 0_{27 \times 27} & \\
& & 0_{16 \times 16} & & & \\
& & & & & \\
& & & 1_{1 \times 1} & & \\
& & & & \mathbf{C} \cdot 1_{10 \times 10} & \\
& & & & & 0_{16 \times 16}
\end{array}\right) \text {; }
\end{aligned}
$$

thus, there are 32 vanishing and 22 strictly positive real eigenvalues.

It is then clear that the mass spectrum of $\mathcal{O}_{n o n-B P S, Z=0, J_{3}^{\oplus}}$ splits under $S O(10)=$ $\frac{m . c . s .\left(E_{6(-14)}\right)}{U(1)}$ as follows: there are 32 massless non-BPS, $Z=0$ "modes" fitting the 32 real degrees of freedom corresponding to the complex $\mathbf{1 6}_{1}$ of $S O(10)$ in the r.h.s. of decomposition (5.11), and $20+2=22$ massive non-BPS, $Z=0$ "modes" fitting the remaining real degrees of freedom corresponding to the complex $\mathbf{1 0}_{-2}$ and $\mathbf{1}_{4}$ of $S O(10)$. The resulting interpretation of the decomposition (5.11) is

$$
\mathbf{2 7} \longrightarrow\left(\begin{array}{c}
\mathbf{1 6}_{1} \\
32 \text { massless }
\end{array}\right)+\left(\begin{array}{c}
\mathbf{1 0}_{-2}+\mathbf{1}_{4} \\
20+2 \text { massive }
\end{array}\right) .
$$

By looking at the form of the non-BPS, $Z=0$ solution (5.14) in "flat" coordinates, it is then easy to realize that the $\mathbf{1 6}_{1}$ of $S O(10)$ remains massless at regular non-BPS, $Z=0$ critical points because it does not couple to the $S O(10)$-singlet in the representation decomposition (5.12). 
The results obtained above for the $\frac{E_{7(-25)}}{E_{6} \otimes U(1)}$-based "magical" $N=2, d=4$ symmetric MESGT can be immediately extended to all the other three "magical" $N=2, d=4$ symmetric MESGTs (corresponding to cases $I V, V$ and $V I$ of Tables 2 and 3 ) as follows.

In all "magical" $N=2, d=4$ MESGTs the complex representation $R_{H_{0}}$ of $H_{0}$ decomposes under $\widetilde{h}^{\prime}=\frac{m . c . s .(\widetilde{H})}{U(1)}$ in the following way (see Eq. (4.2.2.2) ):

$$
R_{H_{0}} \longrightarrow \mathcal{W}_{\widetilde{h}^{\prime}}+\mathcal{Y}_{\widetilde{h}^{\prime}}+\mathbf{1}_{\mathbb{C}}
$$

where in the r.h.s. the complex $\widetilde{h}^{\prime}$-singlet and the complex non-singlet representations $\mathcal{W}_{\widetilde{h}^{\prime}}$ and $\mathcal{Y}_{\widetilde{h}^{\prime}}$ of $\widetilde{h}^{\prime}$ appear. Correspondingly, the decomposition of the $H_{0}$-invariant representation $\left(R_{H_{0}}\right)^{3}$ in terms of representations of $\widetilde{h}^{\prime}$ reads (see Eq. (4.2.2.4))

$$
\left(R_{H_{0}}\right)^{3} \longrightarrow\left(\mathcal{W}_{\widetilde{h}^{\prime}}\right)^{2} \mathcal{Y}_{\widetilde{h}^{\prime}}+\left(\mathcal{Y}_{\widetilde{h}^{\prime}}\right)^{2} \mathbf{1}_{\mathbb{C}}
$$

Let us now recall that $\operatorname{dim}_{\mathbb{R}} R_{H_{0}}=2 n_{V}$ and $\operatorname{dim}_{\mathbb{R}} \mathbf{1}_{\mathbb{C}}=2$, and let us define

$$
\left.\begin{array}{l}
\operatorname{dim}_{\mathbb{R}} \mathcal{W}_{\widetilde{h}^{\prime}} \equiv \mathbf{W}_{\widetilde{h}^{\prime}} ; \\
\operatorname{dim}_{\mathbb{R}} \mathcal{Y}_{\widetilde{h}^{\prime}} \equiv \mathbf{Y}_{\widetilde{h}^{\prime}} ;
\end{array}\right\}: \mathbf{W}_{\widetilde{h}^{\prime}}+\mathbf{Y}_{\widetilde{h}^{\prime}}+2=2 n_{V}
$$

Thus, it can generally be stated that the mass spectrum along $\mathcal{O}_{n o n-B P S, Z=0}$ of all "magical" $N=2, d=4$ symmetric MESGTs splits under $\widetilde{h}^{\prime}=\frac{\text { m.c.s. }(\widetilde{H})}{U(1)}$ as follows:

- the mass "modes" fitting the $\mathbf{W}_{\widetilde{h}^{\prime}}$ real degrees of freedom corresponding to the complex $\left(U(1)\right.$-charged) non- $\widetilde{h}^{\prime}$-singlet representation $\mathcal{W}_{\tilde{h}^{\prime}}$ (which does not couple to the complex $\widetilde{h}^{\prime}$-singlet in the $H_{0}$-invariant decomposition (5.21) ) remain massless;

- the mass "modes" fitting the $\mathbf{Y}_{\widetilde{h}^{\prime}}+2$ real degrees of freedom corresponding to the complex $\left(U(1)\right.$-charged) non- $\widetilde{h}^{\prime}$-singlet representation $\mathcal{Y}_{\widetilde{h}^{\prime}}$ and to the $(U(1)$-charged) $\widetilde{h}^{\prime}$-singlet $\mathbf{1}_{\mathbb{C}}$ all become massive.

The resulting interpretation of the decomposition (5.20) is

$$
R_{H_{0}} \longrightarrow\left(\begin{array}{c}
\mathcal{W}_{\widetilde{h}^{\prime}} \\
\mathbf{W}_{\widetilde{h}^{\prime}} \text { massless }
\end{array}\right)+\left(\begin{array}{c}
\mathcal{Y}_{\widetilde{h}^{\prime}}+\mathbf{1}_{\mathbb{C}} \\
\mathbf{Y}_{\widetilde{h}^{\prime}}+2 \text { massive }
\end{array}\right)
$$

The interpretations (15.19) and (15.23) show that, even though the complex representations $\mathcal{W}_{\widetilde{h}^{\prime}}, \mathcal{Y}_{\widetilde{h}^{\prime}}$ and $\mathbf{1}_{\mathbb{C}}$ of $\widetilde{h}^{\prime}$ are charged with respect to the explicit factor $U(1)$ always appearing in $\widetilde{h}$, this fact does not affect in any way the splitting of the non-BPS, $Z=0$ mass "modes".

It is worth pointing out that in the $N=2,6, d=4$ SUGRAs based on $\frac{S O^{*}(12)}{U(6)}$ (see Subsubsects. 4.3.1 and 4.3.3) the splitting of the critical non-BPS, $Z=0$ mass spectrum obtained by the above general analysis is in agreement with the results of the $N=6$ analysis of the $\frac{1}{6}$-BPS solutions, yielding 7 massive vector multiplets and 4 massless hypermultiplets [70]. 
The critical mass spectra of the irreducible sequence $\frac{S U(1,1+n)}{U(1) \otimes S U(1+n)}$ and of the reducible sequence $\frac{S U(1,1)}{U(1)} \otimes \frac{S O(2,2+n)}{S O(2) \otimes S O(2+n)}$ are treated in Appendices I and II, respectively.

Generally, the Hessian $\mathbf{H}^{V_{B H}}$ at regular $N=2$, non-BPS critical points of $V_{B H}$ exhibits the following features: it does not have "repelling" directions (i.e. strictly negative real eigenvalues), it has a certain number of "attracting" directions (related to strictly positive real eigenvalues), but it is also characterized by some "flat" directions, i.e. by some vanishing eigenvalues, corresponding to massless non-BPS "modes".

In order to establish whether the considered $N=2$, non-BPS citical points of $V_{B H}$ are actually attractors in a strict sense, i.e. whether they actually are stable minima of $V_{B H}$ in the scalar manifold, one has to proceed further with Kähler-covariant differentiation of $V_{B H}$, dealing (at least) with third and higher-order derivatives. Tripathy and Trivedi [17] presented a charge-dependent statement about the stability of $N=2$, non-BPS critical points in the case $Z \neq 0$ for cubic prepotentials, which would be interesting to check and interpret for $N=2, d=4$ symmetric MESGTs in the "representation decomposition approach" exploited above.

We leave the detailed analysis of the issue of stability of both classes of regular nonBPS critical points $\left(Z \neq 0\right.$ and $Z=0$ ) of $V_{B H}$ in $N=2, d=4$ (symmetric) MESGTs for future work.

\section{Conclusion}

In the present work we have classified the regular solutions of the $N=2, d=4$ extremal black hole attractor equations for homogeneous symmetric special Kähler geometries. For rank-3 symmetric manifolds $\frac{G}{H=H_{0} \otimes U(1)}$ of MESGTs defined by Jordan algebras of degree three such solutions exist in three distinct classes, one $\frac{1}{2}$-BPS and the other two non-BPS, one of which corresponds to vanishing central charge $Z=0$.

We have also shown that these three classes of solutions are in one to one correspondence with the non-degenerate charge orbits of the actions of the duality groups $G$ on the corresponding charge spaces that were classified in Sect. 3.

The non-BPS, $Z=0$ class of regular solutions has no analogue in $d=5$, where a similar classification has been recently given [27.

For the considered rank-3 symmetric manifolds the tensor $C_{i j k}$ of special Kähler geometry is covariantly constant, and in "flat" coordinates it is proportional to the numeric symmetric tensor $d_{I J K}$ of the real special geometry of $N=2, d=5$ MESGTs [37, 38, 39, 62.

The rank-1 family of symmetric manifolds (case $I$ of Table 3 ) has $C_{i j k}=0$. It has only two classes of regular solutions to the attractor equations: one $\frac{1}{2}$-BPS and one non-BPS with $Z=0$. 
For all the rank-3 symmetric spaces the classical black hole entropy is given by the Bekenstein-Hawking entropy-area formula 36]

$$
S_{B H}=\frac{A_{H}}{4}=\pi \sqrt{\left|I_{4}\right|}
$$

where

$$
\left.V_{B H}\right|_{\partial V_{B H}=0}=\sqrt{\left|I_{4}\right|}
$$

and $I_{4}$ is the quartic invariant of the charge vector in the considered non-degenerate charge orbit. For the $\frac{1}{2}$-BPS and non-BPS $Z=0$ classes $I_{4}>0$, while the non-BPS $Z \neq 0$ class has $I_{4}<0$.

For the family of rank-1 homogeneous symmetric manifolds the invariant is instead quadratic; it is positive for $\frac{1}{2}$-BPS orbits and negative for the non-BPS ones (see Appendix I).

We also investigated the (splittings of the) mass spectra of the theory along the three species of classes of non-degenerate charge orbits, finding an agreement with a result recently obtained by Tripathy and Trivedi [17, holding true for generic cubic holomorphic prepotentials and for non-BPS $Z \neq 0$ critical points of $V_{B H}$.

In order to proceed further, it would be interesting to extend the analysis performed in the present work to non-symmetric special Kähler spaces with cubic (holomorphic) prepotentials. A proper subclass of such manifolds is given by the homogeneous non-symmetric spaces described in [41]. We should stress that all special Kähler cubic geometries have an uplifting to five dimensions.

In [71] it was shown that the $N=2, d=5$ "magical" MESGTs defined by $J_{3}^{\mathbb{C}}, J_{3}^{\mathbb{H}}$ and $J_{3}^{\mathbb{Q}}$ are simply the "lowest" members of three infinite families of unified $N=2, d=5$ MESGTs defined by Lorentzian Jordan algebras of degree $>3$. The scalar manifolds of such theories are not homogeneous except for the "lowest" members. It would be interesting to extend the analysis of [27] and of the present work to these theories in five dimensions and to their descendants in four dimensions, respectively.

Another direction for further investigations could be the analysis of non-cubic (holomorphic) prepotentials, corresponding to more general special Kähler geometries, such as the ones of the moduli spaces of Calabi-Yau compactifications.

Finally, another possible extension of the present work might deal with higher $N(=$ 3,4) SUGRAs coupled to matter multiplets, which are known to have BPS attractor solutions [10, 11, 12].

\section{Acknowledgements}

S. F. would like to thank L. Andrianopoli and R. D'Auria for discussions. A. M. would like to thank the Department of Physics, Theory Unit Group at CERN for its kind hospitality during the completion of the present paper. 
The work of S.B. has been supported in part by the European Community Human Potential Program under contract MRTN-CT-2004-005104 "Constituents, fundamental forces and symmetries of the universe".

The work of S.F. has been supported in part by the European Community Human Potential Program under contract MRTN-CT-2004-005104 "Constituents, fundamental forces and symmetries of the universe", in association with INFN Frascati National Laboratories and by D.O.E. grant DE-FG03-91ER40662, Task C.

The work of M.G was supported in part by the National Science Foundation under grant number PHY-0245337 and PHY-0555605. Any opinions, findings and conclusions or recommendations expressed in this material are those of the authors and do not necessarily reflect the views of the National Science Foundation.

The work of A.M. has been supported by a Junior Grant of the "Enrico Fermi" Center, Rome, in association with INFN Frascati National Laboratories.

\section{A Appendix I The sequence $\frac{S U(1,1+n)}{U(1) \otimes S U(1+n)}$}

The $N=2, d=4$ symmetric MESGTs with scalar manifolds belonging to the infinite sequence of irreducible rank-1 homogeneous symmetric special Kähler manifolds $\frac{S U(1,1+n)}{U(1) \otimes S U(1+n)}$ feature another fundamental property: they all have a quadratic prepotential, and thus globally

$$
C_{i j k}=0
$$

Consequently, in such a case the attractor eqs. (2.12) acquire the trivial form

$$
\bar{Z} D_{i} Z=0, \forall i=1, \ldots, n_{V}
$$

and therefore they admit only two classes of solutions:

1. $\frac{1}{2}$-BPS solutions:

$$
\begin{aligned}
& Z \neq 0, \quad D_{i} Z=0, \forall i=1, \ldots, n_{V} \\
& V_{B H, \frac{1}{2}-B P S, I}=|Z|_{\frac{1}{2}-B P S, I}^{2} .
\end{aligned}
$$

2. non-BPS, $Z=0$ solutions:

$$
\begin{aligned}
& Z=0, \quad D_{i} Z \neq 0, \text { for some } i \in\left\{1, \ldots, n_{V}\right\} \\
& V_{B H, \text { non-BPS,Z=0,I}}=\left[G^{i \bar{i}} D_{i} Z \overline{D_{\bar{i}}} \bar{Z}\right]_{n o n-B P S, Z=0, I} .
\end{aligned}
$$

No non-BPS, $Z \neq 0$ solutions exist at all. 
Recall that in this case $n_{V}=n+1$. Since the little group of the $S U(n+1)$-vector $D_{i} Z$ is $S U(n)$, it is then clear that the non-BPS, $Z=0$ solutions (AI.4) are $S U(n)$ invariant. On the other hand, since in $\frac{1}{2}$-BPS solutions (AI.3) the vector $D_{i} Z$ vanishes, the symmetry of the solution gets enhanced to $S U(n+1)$. Consequently, the two classes of non-degenerate charge orbits of the case at hand respectively reads (see Table 3 ):

$$
\begin{aligned}
& \mathcal{O}_{\frac{1}{2}-B P S, I}=\frac{S U(1, n+1)}{S U(n+1)} \\
& \mathcal{O}_{n o n-B P S, Z=0, I}=\frac{S U(1, n+1)}{S U(1, n)} .
\end{aligned}
$$

Such two species of orbits are classified by a quadratic invariant, which in "flat" coordinates reads

$$
\mathbf{I}_{2}=|Z|^{2}-\sum_{I=1}^{n_{V}}\left|D_{I} Z\right|^{2}
$$

One thus gets that

$$
\begin{aligned}
& V_{B H, \frac{1}{2}-B P S, I}=|Z|_{\frac{1}{2}-B P S, I}^{2}=\mathbf{I}_{2, \frac{1}{2}-B P S}>0 ; \\
& V_{B H, \text { non-BPS }, Z=0, I}=\sum_{I=1}^{n_{V}}\left|D_{I} Z\right|_{\frac{1}{2}-B P S, I}^{2}=-\mathbf{I}_{2, \text { non-BPS }, Z=0}>0 .
\end{aligned}
$$

For what concerns the critical mass spectrum, $\frac{1}{2}$-BPS case is well known to be always stable for all $N=2, d=4$ MESGTs, and it has been treated in Sect. 5. Considering the non-BPS, $Z=0$ critical mass spectrum and using Eq. (AI.1), Eqs. (5.2) and (5.3) immediately yield the following elements of the $(2 n+2) \times(2 n+2)$ critical Hessian $\mathbf{H}_{\text {non-BPS,Z=0,I}}^{V_{B H}}$ :

$$
\begin{aligned}
& \mathcal{M}_{I J, \text { non }-B P S, Z=0, I}=0 \\
& \mathcal{N}_{I \bar{J}}=2\left[D_{I} Z \bar{D} \bar{J}_{\bar{Z}}\right]_{n o n-B P S, Z=0, I}
\end{aligned}
$$

Since the $(n+1) \times(n+1)$ Hermitian matrix $\left[D_{I} Z \bar{D} \bar{J}_{\bar{J}}\right]_{n o n-B P S, Z=0, I}$ has rank 1 , one immediately gets that the $(2 n+2) \times(2 n+2)$ Hermitian matrix $\mathbf{H}_{n \text { on }-B P S, Z=0, I}^{V_{B H}}$ has rank 2 : it has 2 strictly positive and $2 n$ vanishing real eigenvalues. This is the general splitting pattern of the mass spectrum along $\mathcal{O}_{n o n-B P S, Z=0}$ in the irreducible $N=2, d=4$ symmetric $\frac{S U(1,1+n)}{U(1) \otimes S U(1+n)}$-based MESGTs.

\section{B Appendix II The sequence $\frac{S U(1,1)}{U(1)} \otimes \frac{S O(2,2+n)}{S O(2) \otimes S O(2+n)}$}

The case $I I$ of Tables 2 and 3 is given by the sequence of $N=2, d=4$ symmetric MESGTs based on rank-3 reducible homogeneous symmetric special Kähler manifolds $\frac{S U(1,1)}{U(1)} \otimes \frac{S O(2,2+n)}{S O(2) \otimes S O(2+n)}(n \in \mathbb{N} \cup\{0\})$ with real dimension $2(n+3)$. The main difference 
with respect to the four "magical" $N=2, d=4$ symmetric MESGTs (cases $I I I-V I$ ) and the sequence $\frac{S U(1,1+n)}{U(1) \otimes S U(1+n)}$ (case $I$ ) treated above is the fact that for case $I I$ the scalar manifold is reducible, being the direct product of two distinct manifolds.

In this case $G=S U(1,1) \otimes S O(2,2+n)$ and $H_{0}=S O(2) \otimes S O(2+n)$. However, the holomorphic prepotential $F$ has at most an $S O(n+1)$ manifest compact symmetry. For instance, in a suitable basis of special coordinates, $F$ may be written as follows:

$$
F(t)=t^{1}\left[\left(t^{2}\right)^{2}-\left(t^{3}\right)^{2}-\sum_{i=4}^{n+3}\left(t^{i}\right)^{2}\right],
$$

which can be identified with the norm form of the underlying generic family of non-simple Jordan algebras (see Sect. 3).

Beside the manifest overall symmetry $S O(1, n+1)$ (which is clearly related to its 5-dimensional origin, since $\left.S O(1, n+1)=\frac{\widehat{H}=G_{5}}{S O(1,1)}\right)$, it should be remarked the full factorization of the holomorphic cubic function (AII.1) in linear and quadratic components, due to the reducibility of the scalar manifold, which follows from the fact that the underlying Jordan algebras are not simple.

A particular, noteworthy case is given by the $n=0$ element $I I_{0}$ which is the $N=2$, $d=4$ symmetric MESGT with $n_{V}=3$-moduli usually called stu model [42]. Its scalar manifold and prepotential are given by Eq. (2.4).

At this point, it is worth recalling that there are actually two other typologies of $N=$ $2, d=4$ symmetric MESGTs, corresponding to the following cosets and prepotentials (in suitable systems of special coordinates):

$$
\begin{aligned}
& V I I: \frac{S U(1,1)}{U(1)}, r=1, F(t)=\frac{1}{3} t^{3} \\
& V I I I: \frac{S U(1,1)}{U(1)} \otimes \frac{S O(2,1)}{S O(2)}=\left(\frac{S U(1,1)}{U(1)}\right)^{2}, r=2, F(s, t)=s t^{2} .
\end{aligned}
$$

Notice that, even though they share the same coset structure, the cases $I_{0}$ and $V I I$ correspond to different prepotential functions: while $I_{0}$ is the $n=0,1$-modulus element of the sequence $I$ with quadratic prepotential, the 1-modulus case VII can actually be obtained from the $s t u$ model $I I_{0}$ by putting $s=t=u$ (and rescaling everything by $\sqrt[3]{3}$ ). On the other hand, the 2-moduli case VIII can also be obtained from the stu model $I I_{0}$, e.g. by putting $t=u$, or also by putting $n=-1$ in sequence $I I$.

Summarizing, the cases VII and VIII can both be obtained as consistent truncations of the stu model, simply by identifying some or all moduli, and consequently destroying the related triality symmetry.

While the $n_{V}=2$-moduli case VIII has the usual three classes of $N=2$ critical solutions, the $n_{V}=1$-moduli case $V I I$ has only $\frac{1}{2}$-BPS and non-BPS, $Z \neq 0$ solutions, as it can be easily seen by considering the structure of the attractor eqs. (2.12) for 
such cases. Models $I I_{0}(s t u), V I I$ and VIII are important also because in such highly symmetric cases with a few moduli one can actually manage to analytically solve the attractor eqs. (2.12) for the purely charge-dependent critical values of the moduli [69].

In order to solve the $N=2$ attractor eqs. (2.12) for the $\frac{S U(1,1)}{U(1)} \otimes \frac{S O(2,2+n)}{S O(2) \otimes S O(2+n)}$-based $N=2, d=4$ symmetric MESGTs, it is once again crucially convenient to switch to "flat" $I$-coordinates, in which the $N=2$ attractor eqs. are given by Eqs. (2.17):

$$
\begin{aligned}
& \partial_{I} V_{B H}=0 \Leftrightarrow 2 \bar{Z} D_{I} Z=-i C_{I J K} \delta^{J \bar{J}} \delta^{K \bar{K}} \bar{D} \bar{J} \overline{Z D} \bar{K}_{\bar{Z}} \bar{Z}=0, \\
& \forall I=0,1, \ldots, n_{V}-1=n+2 .
\end{aligned}
$$

In such a "flat" $I$-coordinate system, even though it cannot be written as the third partial derivative tensor of the prepotential (since no $F$ exists at all: see Footnote 6 ), the rank-3 symmetric tensor $C_{I J K}$ becomes very simple, since its unique non-vanishing components are simply determined by the norm forms of the underlying reducible family of Jordan algebras of degree three:

$$
C_{I J K}=C_{0 \widehat{J} \widehat{K}}=C_{0} \delta_{\widehat{J} \widehat{K}}, \quad C_{0} \in \mathbb{C}_{0},
$$

where the "hatted" "flat" indices' range is $\{1, \ldots, n+2\}$ and $\delta_{\widehat{J} \hat{K}}$ is the $S O(n+2)$-invariant Euclidean metric. Consequently, Eqs. AII.4 split as follows:

$$
\left\{\begin{array}{l}
2 \bar{Z} D_{0} Z=-i C_{0} \delta^{\overline{\widehat{J} \widehat{K}}} \bar{D} \overline{\widehat{J}} \overline{Z D} \overline{\widehat{K}} \bar{Z}=-i C_{0} \sum_{\widehat{J}=1}^{n+2}(\bar{D} \overline{\widehat{J}} \bar{Z})^{2}=0 \\
2 \bar{Z} D_{\widehat{I}} Z=-i C_{0} \bar{D}_{\overline{0}} \overline{Z D} \overline{\bar{I}} \bar{Z}=0 .
\end{array}\right.
$$

Thus, it is immediate to obtain the three classes of regular solutions of $N=2$ extremal black hole attractor eqs. (AII.6) in "flat" $I$-coordinates for the $\frac{S U(1,1)}{U(1)} \otimes \frac{S O(2,2+n)}{S O(2) \otimes S O(2+n)}$ based $N=2, d=4$ symmetric MESGTs:

$\frac{1}{2}$-BPS class:

$$
\begin{aligned}
& Z \neq 0, \quad D_{0} Z=0=D_{\widehat{I}} Z, \quad \forall \widehat{I}=1, \ldots, n+2 ; \\
& V_{B H, \frac{1}{2}-B P S, I I}=|Z|_{\frac{1}{2}-B P S, I I}^{2} .
\end{aligned}
$$

Non-BPS, $Z \neq 0$ class (recall Eq. $(\sim)$ in Footnote 15):

$$
\begin{aligned}
& Z \neq 0, \quad D_{0} Z \neq 0, \quad D_{1} Z \neq 0, \quad D_{\breve{I}} Z=0, \breve{I} \in\{2, \ldots, n+2\} ;
\end{aligned}
$$

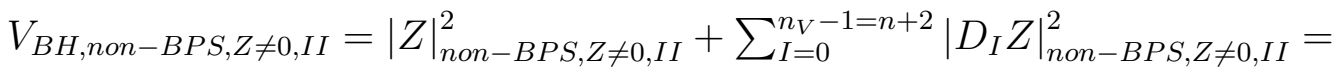

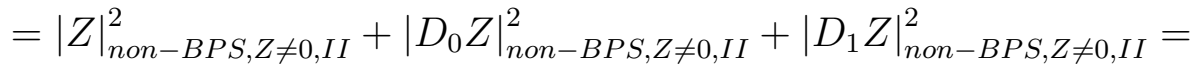

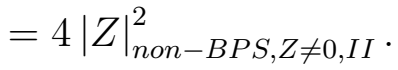


Non-BPS, $Z=0$ classes.

In this case, there are two distinct sets of independent regular non-BPS, $Z=0$ solutions to $N=2$ extremal black hole attractor eqs. (AII.6); they read:

$$
\begin{aligned}
& (\text { non-BPS })_{1}:\left\{\begin{array}{l}
Z=0, \quad D_{0} Z=0, \quad D_{1} Z= \pm i D_{2} Z, D_{\widehat{I}} Z=0, \quad \forall \widehat{I}=3, \ldots, n+2 ; \\
V_{B H, \text { non }-B P S, Z=0, I I, 1}=2\left|D_{1} Z\right|_{\text {non-BPS,Z=0,II,1 }}^{2}
\end{array}\right. \\
& (\text { (non-BPS })_{2}:\left\{\begin{array}{l}
Z=0, \quad D_{0} Z \neq 0, \quad D_{\widehat{I}} Z=0, \quad \forall \widehat{I}=1, \ldots, n+2 ; \\
V_{B H, \text { non }-B P S, Z=0, I I, 2}=\left|D_{0} Z\right|_{\text {non-BPS,Z=0,II,2 }}^{2} .
\end{array}\right.
\end{aligned}
$$

Let us analyze the overall symmetry of the solutions (AII.7)-(AII.10).

For the $\frac{1}{2}$-BPS case $D_{0} Z=0=D_{\widehat{I}} Z$, thus the compact symmetry is $S O(2) \otimes S O(2+n)$. This is also the case of the type 2 of non-BPS $Z=0$ class (see Eq. (AII.10). For the type 1 of non-BPS $Z=0$ class $\left(D_{1} Z\right)^{2}+\left(D_{2} Z\right)^{2}=0$ and $D_{0} Z=0$, therefore the compact symmetry is $S O(2) \otimes S O(2) \otimes S O(n)$. Finally, for the non-BPS $Z \neq 0$ class $D_{0} Z \neq 0$ and $D_{1} Z \neq 0$, thence the compact symmetry is $S O(1+n)$.

The cases $\frac{1}{2}$-BPS, non-BPS $Z \neq 0$ and non-BPS $Z=0$ type 1 correspond to the three classes of non-degenerate orbits of $N=2, d=4$ symmetric $\frac{S U(1,1)}{U(1)} \otimes \frac{S O(2,2+n)}{S O(2) \otimes S O(2+n)}$-based MESGTs given in Table 3.

The case non-BPS $Z=0$ type 2 corresponds instead to the class of orbits $\frac{S U(1,1) \otimes S O(2,2+n)}{S O(2) \otimes S O(2+n)}$, isomorphic to (but physically distinct from) the $\frac{1}{2}$-BPS class. Indeed, even though in the case at hand $\mathcal{O}_{n o n-B P S, Z=0}$ and $\mathcal{O}_{\frac{1}{2}-B P S}$ have the same formal coset expression, they actually correspond to the tips of the two separated branches of the disconnected manifold $\frac{S U(1,1) \otimes S O(2,2+n)}{S O(2) \otimes S O(2+n)}$, classified by the sign of the quantity $\widetilde{\mathbf{I}}_{2} \equiv|Z|^{2}-\left|D_{0} Z\right|^{2}$ :

$$
\begin{aligned}
& \widetilde{\mathbf{I}}_{2, \frac{1}{2}-B P S, I I}=|Z|_{\frac{1}{2}-B P S, I I}^{2}>0 \\
& \widetilde{\mathbf{I}}_{2, \text { non-BPS,Z=0,II,2}}=-\left|D_{0} Z\right|_{\text {non }-B P S, Z=0, I I, 2}^{2}<0 .
\end{aligned}
$$

For such classes of critical points the quartic invariant reads [12]

$$
I_{4, I I}=\left(\widetilde{\mathbf{I}}_{2, I I}\right)^{2}>0
$$

the two cases corresponding to $\widetilde{\mathbf{I}}_{2, I I} \gtrless 0$ (see Eq. (AII.11) $)$.

This can also be seen in the $n=0$ element $I I_{0}$ of the sequence $I I$ being treated, i.e. in the stu model, where explicit calculations are feasible [69]. 
Indeed, by setting $n=0$ in the third row of Table 3 , one obtains the three classes of non-degenerate orbits of the stu model $I I_{0}$ (the two $Z=0$ orbits coincide in this case):

$\frac{1}{2}$-BPS class $\left(H_{0}=(U(1))^{2}\right)$ :

$$
\mathcal{O}_{\frac{1}{2}-B P S, s t u}=\frac{G}{H_{0}}=\frac{(S U(1,1))^{3}}{(U(1))^{2}} ;
$$

Non-BPS, $Z=0$ class $\left(\widetilde{H}=H_{0}=(U(1))^{2}\right)$ :

$$
\mathcal{O}_{\text {non-BPS }, Z=0, s t u}=\frac{G}{H_{0}}=\frac{(S U(1,1))^{3}}{(U(1))^{2}} ;
$$

Non-BPS, $Z \neq 0$ class $\left(\widehat{H}=(S O(1,1))^{2}\right)$ :

$$
\mathcal{O}_{n o n-B P S, Z \neq 0, s t u}=\frac{G}{\widehat{H}}=\frac{(S U(1,1))^{3}}{(S O(1,1))^{2}} .
$$

The orbits $\mathcal{O}_{\frac{1}{2}-B P S, s t u}$ and $\mathcal{O}_{\text {non-BPS,Z=0,stu }}$, despite having the same coset expression, correspond to different values of the quantity $|Z|^{2}-\left|D_{s} Z\right|^{2}$. Indeed, it can be explicitly computed [69] that:

$\frac{1}{2}-\mathrm{BPS}$ orbit $\mathcal{O}_{\frac{1}{2}-B P S, s t u}$ :

$$
|Z|_{\frac{1}{2}-B P S, s t u}^{2}-\left|D_{s} Z\right|_{\frac{1}{2}-B P S, s t u}^{2}=|Z|_{\frac{1}{2}-B P S, s t u}^{2}>0
$$

Non-BPS, $Z=0$ orbit $\mathcal{O}_{n o n-B P S, Z=0, s t u}$ (recall Eq. (4.2.2.7)):

$$
\begin{aligned}
& |Z|_{\text {non-BPS,Z=0,stu }}^{2}-\left|D_{s} Z\right|_{\text {non-BPS,Z=0,stu }}^{2}= \\
& =-\left|D_{s} Z\right|_{\text {non-BPS,Z=0,stu }}^{2}=-|Z|_{\frac{1}{2}-B P S, \text { stu }}^{2}<0 .
\end{aligned}
$$

Consequently, the orbits $\mathcal{O}_{\frac{1}{2}-B P S, s t u}$ and $\mathcal{O}_{n o n-B P S, Z=0, s t u}$ correspond to two separated branches of a disconnected manifold, classified by the local value of the function $\operatorname{sgn}\left(|Z|^{2}-\left|D_{s} Z\right|^{2}\right)$. Such a result can be easily extended to a generic $n \in \mathbb{N}$, i.e. to a generic element of the sequence $\frac{S U(1,1)}{U(1)} \otimes \frac{S O(2,2+n)}{S O(2) \otimes S O(2+n)}$.

As for the classes of non-degenerate orbits of cases $V I I$ and $V I I I$, they respectively read as follows:

Case VII $(G=S U(1,1))$

$\frac{1}{2}$-BPS class $\left(H_{0}=1\right)$ :

$$
\mathcal{O}_{\frac{1}{2}-B P S, V I I}=\frac{G}{H_{0}}=S U(1,1), \quad I_{4}>0
$$

Non-BPS, $Z \neq 0$ class $(\widehat{H}=1)$ :

$$
\mathcal{O}_{n o n-B P S, Z \neq 0, V I I}=\frac{G}{\widehat{H}}=S U(1,1), \quad I_{4}<0 .
$$


Case VIII $(G=S U(1,1) \otimes S U(1,1))$; it can actually be obtained by putting $n=-1$ in the third row of Table 3 :

$\frac{1}{2}$-BPS class $\left(H_{0}=U(1)\right)$ :

$$
\mathcal{O}_{\frac{1}{2}-B P S, V I I I}=\frac{G}{H_{0}}=S U(1,1) \otimes \frac{S O(2,1)}{S O(2)}=\frac{S U(1,1) \otimes S U(1,1)}{U(1)} ;
$$

Non-BPS, $Z=0$ class $\left(\widetilde{H}=H_{0}=U(1)\right)$ :

$$
\mathcal{O}_{n o n-B P S, Z=0, V I I I}=\frac{G}{H_{0}}=\frac{S U(1,1) \otimes S U(1,1)}{U(1)} ;
$$

Non-BPS, $Z \neq 0$ class $(\widehat{H}=S O(1,1))$ :

$$
\mathcal{O}_{n o n-B P S, Z \neq 0, V I I I}=\frac{G}{\widehat{H}}=\frac{S U(1,1) \otimes S U(1,1)}{S O(1,1)} .
$$

Let us now consider the mass spectrum of the reducible sequence $I I$ at the regular critical points of $V_{B H}$.

From the above analysis, it is clear that for the infinite set of reducible $N=2, d=4$ symmetric MESGTs based on $\frac{S U(1,1)}{U(1)} \otimes \frac{S O(2,2+n)}{S O(2) \otimes S O(2+n)}$, the structure of the Hessian at $\frac{1}{2}$-BPS and non-BPS, $Z=0$ critical points will be the same.

As it was pointed out more than once above, in all $N=2, d=4$ MESGTs (with regular special Kähler geometry of the scalar manifold) the $2 n_{V} \times 2 n_{V}$ real form $\mathbf{H}_{\frac{1}{2}-B P S \text {,real }}^{V_{B H}}$ of the $\frac{1}{2}$-BPS critical Hessian has $2 n_{V}$ strictly positive real eigenvalues, and therefore all $\frac{1}{2}$-BPS critical points of $V_{B H}$ are attractors in a strict sense, i.e. they are stable minima of $V_{B H}$. Consequently, one immediately obtains that in the case of $\frac{S U(1,1)}{U(1)} \otimes \frac{S O(2,2+n)}{S O(2) \otimes S O(2+n)}$ the same holds also for all non-BPS, $Z=0$ critical points of $V_{B H}$ : they all are attractors in a strict sense, i.e. they are stable minima of $V_{B H}$.

Concerning the non-BPS, $Z \neq 0$ mass spectrum, one can finally say that, despite the reducibility of such manifolds, the same analysis performed in Sect. 5 for (irreducible) $N=2, d=4$ "magical" symmetric MESGTs holds also for this case, and Tripathy and Trivedi's result [17 is confirmed.

Thus, it can generally be stated that the mass spectrum along $\mathcal{O}_{n o n-B P S, Z \neq 0}$ of all reducible $N=2, d=4$ symmetric $\frac{S U(1,1)}{U(1)} \otimes \frac{S O(2,2+n)}{S O(2) \otimes S O(2+n)}$-based MESGTs splits under $\widehat{h}=$ m.c.s. $(\widehat{H})=S O(1+n)$ as follows: there are $n_{V}-1=n+2$ massless and $n_{V}+1=n+4$ massive non-BPS, $Z \neq 0$ mass "modes".

Once again, Tripathy and Trivedi's result can also be confirmed by performing explicit calculations in the highly symmetric and manageable case of the stu model ${ }^{16}$ [69].

\footnotetext{
${ }^{16}$ Clearly, since the isolated models VII and VIII are consistent truncations of the stu model, once
} 


\section{References}

[1] S. Ferrara and M. Günaydin, Orbits of Exceptional Groups, Duality and BPS States in String Theory, Int. J. Mod. Phys. A13, 2075 (1998), hep-th/9708025.

[2] C. Hull and P. K. Townsend, Unity of Superstring Dualities, Nucl. Phys. B438, 109 (1995), hep-th/9410167.

[3] M. Günaydin, K. Koepsell and H. Nicolai, Conformal and quasiconformal realizations of exceptional Lie groups, Commun. Math. Phys. 221, 57 (2001), hep-th/0008063.

[4] M. Günaydin, Unitary realizations of U-duality groups as conformal and quasiconformal groups and extremal black holes of supergravity theories, AIP Conf. Proc. 767, 268 (2005), hep-th/0502235.

[5] M. Günaydin, K. Koepsell and H. Nicolai, The Minimal Unitary Representation of $E_{8(8)}$, Adv. Theor. Math. Phys. 5, 923 (2002), hep-th/0109005.

[6] M. Günaydin and O. Pavlyk, Minimal unitary realizations of exceptional U-duality groups and their subgroups as quasiconformal groups, JHEP 0501, 019 (2005), hep-th/0409272.

[7] M. Günaydin and O. Pavlyk, Generalized spacetimes defined by cubic forms and the minimal unitary realizations of their quasiconformal groups, JHEP 0508, 101 (2005), hep-th/0506010.

[8] B. Pioline, BPS black hole degeneracies and minimal automorphic representations, JHEP 0508, 071 (2005), hep-th/0506228.

[9] M. Günaydin, A. Neitzke, B. Pioline and A. Waldron, BPS black holes, quantum attractor flows and automorphic forms, Phys. Rev. D73, 084019 (2006), hep-th/0512296.

[10] S. Ferrara, R. Kallosh and A. Strominger, $N=2$ Extremal Black Holes, Phys. Rev. D52, 5412 (1995), hep-th/9508072.

[11] S. Ferrara and R. Kallosh, Supersymmetry and Attractors, Phys. Rev. D54, 1514 (1996), hep-th/9602136.

[12] S. Ferrara and R. Kallosh, Universality of Supersymmetric Attractors, Phys. Rev. D54, 1525 (1996), hep-th/9603090.

the critical mass spectrum for the stu model is known, one can obtain the critical mass spectra for the models VII and VIII. Such results will be presented elsewhere [69]. 
[13] A. Strominger, Macroscopic Entropy of $N=2$ Extremal Black Holes, Phys. Lett. B383, 39 (1996), hep-th/9602111.

[14] S. Ferrara, G. W. Gibbons and R. Kallosh, Black Holes and Critical Points in Moduli Space, Nucl. Phys. B500, 75 (1997), hep-th/9702103.

[15] K. Goldstein, N. Tizuka, R. P. Jena and S. P. Trivedi, Non-Supersymmetric Attractors, Phys. Rev. D72, 124021 (2005), hep-th/0507096.

[16] R. Kallosh, New Attractors, JHEP 0512, 022 (2005), hep-th/0510024.

[17] P. K. Tripathy and S. P. Trivedi, Non-Supersymmetric Attractors in String Theory, JHEP 0603, 022 (2006), hep-th/0511117.

[18] A. Giryavets, New Attractors and Area Codes, JHEP 0603, 020 (2006), hep-th/0511215.

[19] K. Goldstein, R. P. Jena, G. Mandal and S. P. Trivedi, A C-Function for NonSupersymmetric Attractors, JHEP 0602, 053 (2006), hep-th/0512138.

[20] M. Alishahiha and H. Ebrahim, Non-supersymmetric attractors and entropy function, JHEP 0603, 003 (2006), hep-th/0601016.

[21] R. Kallosh, N. Sivanandam and M. Soroush, The Non-BPS Black Hole Attractor Equation, JHEP 0603, 060 (2006), hep-th/0602005.

[22] B. Chandrasekhar, S. Parvizi, A. Tavanfar and H. Yavartanoo, Non-supersymmetric attractors in $R^{2}$ gravities, JHEP 0608, 004 (2006), hep-th/0602022.

[23] J. P. Hsu, A. Maloney and A. Tomasiello, Black Hole Attractors and Pure Spinors, hep-th/0602142.

[24] B. Sahoo and A. Sen, Higher-derivative corrections to non-supersymmetric extremal black holes in $N=2$ supergravity, hep-th/0603149.

[25] M. Alishahiha and H. Ebrahim, New attractor, Entropy Function and Black Hole Partition Function, hep-th/0605279.

[26] S. Ferrara and R. Kallosh, On $N=8$ Attractors, Phys. Rev. D73, 125005 (2006), hep-th/0603247.

[27] S. Ferrara and M. Günaydin, Orbits and attractors for $N=2$ Maxwell-Einstein supergravity theories in five dimensions, hep-th/0606108.

[28] E. Cremmer and B. Julia, The SO(8) Supergravity, Nucl. Phys. B159, 141 (1979). 
[29] E. Cartan, Euvres complètes (Editions du Centre National de la Recherche Scientifique, Paris, 1984).

[30] M. Cvetic and D. Youm, Dyonic BPS Saturated Black Holes of Heterotic String on a Six Torus, Phys. Rev. D53, 584 (1996), hep-th/9507090 $\diamond$ M. Cvetic and A. A. Tseytlin, Solitonic Strings and BPS Saturated Dyonic Black Holes, Phys. Rev. D53, 5619 (1996); Erratum-ibid. D55, 3907 (1997), hep-th/9512031 $\diamond \mathrm{M}$. Cvetic and C. M. Hull, Black Holes and U-Duality, Nucl. Phys. B480, 296 (1996), hep-th/9606193.

[31] L. Andrianopoli, R. D'Auria, S. Ferrara, P. Frè and M. Trigiante, E $E_{7(7)}$ duality, BPS black-hole evolution and fixed scalars, Nucl. Phys. B509, 463 (1998), hep-th/9707087.

[32] S. Ferrara, C. A. Savoy and B. Zumino, General Massive Multiplets in Extended Supersymmetry, Phys. Lett. B100, 393 (1981).

[33] C. Bloch and A. Messiah, Nucl. Phys. 39, 95 (1962).

[34] B. Zumino, J. Math. Phys. 3, 1055 (1962).

[35] S. Ferrara and J. M. Maldacena, Branes, central charges and U-duality invariant BPS conditions, Class. Quant. Grav. 15, 749 (1998), hep-th/9706097.

[36] J. D. Bekenstein, Phys. Rev. D7, 2333 (1973) $\diamond$ S. W. Hawking, Phys. Rev. Lett. 26, 1344 (1971); in C. DeWitt, B. S. DeWitt, Black Holes (Les Houches 1972) (Gordon and Breach, New York, 1973) $\diamond$ S. W. Hawking, Nature 248, 30 (1974) $\diamond$ S. W. Hawking, Comm. Math. Phys. 43, 199 (1975).

[37] M. Günaydin, G. Sierra and P. K. Townsend, Exceptional Supergravity Theories and the Magic Square, Phys. Lett. B133, 72 (1983).

[38] M. Günaydin, G. Sierra and P. K. Townsend, The Geometry of $N=2$ MaxwellEinstein Supergravity and Jordan Algebras, Nucl. Phys. B242, 244 (1984).

[39] M. Günaydin, G. Sierra and P. K. Townsend, Gauging the D = 5 Maxwell-Einstein Supergravity Theories: More on Jordan Algebras, Nucl. Phys. B253, 573 (1985).

[40] E. Cremmer and A. Van Proeyen, Classification of Kähler Manifolds in $N=2$ Vector Multiplet Supergravity Couplings, Class. Quant. Grav. 2, 445 (1985).

[41] B. de Wit, F. Vanderseypen and A. Van Proeyen, Symmetry Structures of Special Geometries, Nucl. Phys. B400, 463 (1993), hep-th/9210068. 
[42] K. Behrndt, R. Kallosh, J. Rahmfeld, M. Shmakova and W. K. Wong, STU Black Holes and String Triality, Phys. Rev. D54, 6293 (1996), hep-th/9608059.

[43] M. J. Duff, String Triality, Black Hole Entropy and Cayley's Hyperdeterminant, hep-th/0601134.

[44] R. Kallosh and A. Linde, Strings, Black Holes and Quantum Information, Phys. Rev. D73, 104033 (2006), hep-th/0602061.

[45] P. Lévay, Stringy Black Holes and the Geometry of the Entanglement, Phys. Rev. D74, 024030 (2006), hep-th/0603136.

[46] H. Freudenthal, Proc. Konink. Ned. Akad. Wetenschap A62, 447 (1959).

[47] B. A. Rozenfeld, Dokl. Akad. Nauk. SSSR 106, 600 (1956) $\diamond$ J. Tits, Mem. Acad. Roy. Belg. Sci. 29, fasc. 3 (1955).

[48] P. Jordan, J. Von Neumann and E. Wigner, On an algebraic generalization of the quantum mechanical formalism, Ann. Math. 35, 29 (1934).

[49] N. Jacobson, Ann. Math. Soc. Coll. Publ. 39 (1968).

[50] M. Günaydin, Exceptional Realizations of Lorentz Group: Supersymmetries and Leptons, Nuovo Cimento A29, 467 (1975).

[51] M. Günaydin, C. Piron and H. Ruegg, Moufang Plane and Octonionic Quantum Mechanics, Comm. Math. Phys. 61, 69 (1978).

[52] A. Ceresole, R. D'Auria and S. Ferrara, The Symplectic Structure of $N=2$ SUGRA and Its Central Extension, Talk given at ICTP Trieste Conference on Physical and Mathematical Implications of Mirror Symmetry in String Theory, Trieste, Italy, 5-9 June 1995, Nucl. Phys. Proc. Suppl. 46 (1996), hep-th/9509160.

[53] A. Ceresole, R. D'Auria, S. Ferrara and A. Van Proeyen, Duality transformations in supersymmetric Yang-Mills theories coupled to supergravity, Nucl. Phys. B444, 92 (1995), hep-th/9502072.

[54] S. Bellucci, S. Ferrara and A. Marrani, On some properties of the Attractor Equations, Phys. Lett. B635, 172 (2006), hep-th/0602161.

[55] S. Bellucci, S. Ferrara and A. Marrani, Supersymmetric Mechanics. Vol.2: The Attractor Mechanism and Space-Time Singularities (LNP 701, Springer-Verlag, Heidelberg, 2006).

[56] L. Andrianopoli, R. D'Auria and S. Ferrara, U Duality and Central Charges in Various Dimensions Revisited, Int. J. Mod. Phys. A13, 431 (1998), hep-th/9612105. 
[57] H. Freudenthal, Adv. Math. 1, 145 (1964).

[58] R. Brown, Groups of Type $E_{7}$, J. Reine Andew. Math. 236, 79 (1969).

[59] J.C. Ferrar, Strictly regular elements in Freudenthal triple systems, Trans. Am. Math. Soc. 174, 313 (1972).

[60] See J. R. Faulkner, Trans. Am. Math. Soc. 167, 49 (1972), and Refs. therein.

[61] K. McKrimmon, Trans. Am. Math. Soc. 139, 495 (1969).

[62] M. Günaydin, G. Sierra and P. K. Townsend, More on $d=5$ Maxwell-Einstein Supergravity: Symmetric Space and Kinks, Class. Quant. Grav. 3, 763 (1986).

[63] K. McCrimmon, A taste of Jordan algebras (Springer Verlag, New York, 2000).

[64] S. Krutelevich, On Orbits in the 56-dimensional module over the group $E_{7}$, preprint Yale University, 2003.

[65] O. Shukuzawa, Explicit classifications of orbits in Jordan algebra and Freudenthal vector space over the exceptional Lie groups, Comm. in Algebra 34, 197 ( 2006).

[66] G.B. Seligman, On the split exceptional Lie algebra $E_{7}$, Yale University, dittoed note 1962. Supplement 1963.

[67] R. Gilmore, Lie Groups, Lie Algebras, and Some of Their Applications (Dover Publications, 2006).

[68] S. Helgason, Differential Geometry, Lie Groups and Symmetric Spaces (Academic Press, New York, 1978).

[69] work in progress.

[70] L. Andrianopoli, R. D'Auria and S. Ferrara, U-invariants, black hole entropy and fixed scalars, Phys. Lett. B403, 12 (1997), hep-th/9703156.

[71] M. Günaydin and M. Zagermann, Unified Maxwell-Einstein and Yang-Mills-Einstein supergravity theories in five dimensions, JHEP 0307, 023 (2003), hep-th/0304109. 